\title{
MINORATIONS SIMULTANÉES DE FORMES LINÉAIRES DE LOGARITHMES DE NOMBRES ALGÉBRIQUES
}

\author{
par Éric Gaudron
}

\begin{abstract}
RÉSumÉ. - Soit $p$ un nombre premier ou $p=\infty$ et $k$ un corps de nombres plongé dans $\mathbf{C}_{p}$. Soit $n \in$ $\mathbf{N} \backslash\{0\}$ et $u_{1}, \ldots, u_{n} \in \mathbf{C}_{p}$ tels que $e^{u_{j}} \in k$ pour tout $j \in\{1, \ldots, n\}$. Soit $\left(\beta_{i, j}\right), 1 \leq i \leq t, 1 \leq j \leq n$, une matrice $t \times n$ à coefficients dans $k$. Soit $\left(\beta_{1,0}, \ldots, \beta_{t, 0}\right) \in k^{t}$. Posons $\Lambda_{i}:=\beta_{i, 0}+\sum_{j=1}^{n} \beta_{i, j} u_{j} \in \mathbf{C}_{p}$ pour tout $i \in\{1, \ldots, t\}$. Nous obtenons des minorations de $\max \left\{\left|\Lambda_{i}\right|_{p} ; 1 \leq i \leq t\right\}$ explicites en tous les paramètres, lorsque ce maximum n'est pas nul. Ces minorations englobent de nombreux résultats antérieurs. La démonstration repose sur la méthode de Baker-Philippon-Waldschmidt, la réduction d'Hirata-Kohno, le procédé de changement de variables de Chudnovsky, repensés avec les outils modernes de la théorie des pentes adéliques (méthode de la section auxiliaire). Au passage, nous montrons comment étendre le lemme de Siegel absolu de Zhang au cadre des fibrés adéliques hermitiens et nous établissons un nouveau lemme de Siegel approché.
\end{abstract}

Abstract (Simultaneous lower bounds for linear forms in logarithms of algebraic numbers)

This work falls within the theory of linear forms in logarithms over a commutative linear algebraic group defined over a number field. We give lower bounds for simultaneous linear forms in logarithms of algebraic numbers, treating both the archimedean and $p$-adic cases. The proof includes Baker's method, Hirata's reduction, Chudnovsky's process of variable change. The novelty is that we integrate into the proof the modern tools of adelic slope theory (auxiliary section method), using a new small values Siegel's lemma.

Table des matières

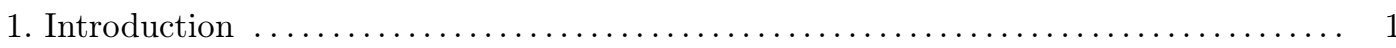

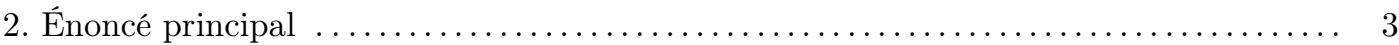

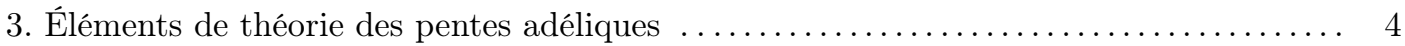

4. Lemmes de Siegel approchés $\ldots \ldots \ldots \ldots \ldots \ldots \ldots \ldots \ldots \ldots \ldots \ldots \ldots \ldots \ldots \ldots \ldots \ldots$

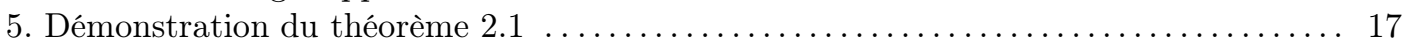

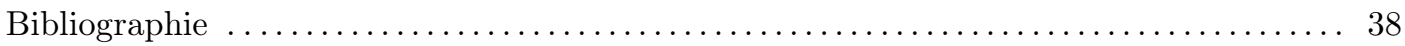

\section{Introduction}

Ce texte présente une minoration simultanée de formes linéaires de logarithmes de nombres algébriques, valide pour un plongement quelconque (complexe ou ultramétrique) du corps de nombres ambiant. En d'autres termes il s'agit de minorer $\max \left\{\left|\Lambda_{i}\right|_{p_{0}} ; 1 \leq i \leq t\right\}$ où $\Lambda_{i}$ est de la forme $\beta_{i, 0}+\sum_{j=1}^{n} \beta_{i, j} u_{j}$ avec $\beta_{i, j}$ et $e^{u_{j}}$ dans un corps de nombres $k$ plongé dans $\mathbf{C}_{p_{0}}$ et $|\cdot|_{p_{0}}$ est une

Version révisée : Lundi 21 mai 2012

Éric Gaudron, Université Grenoble I, Institut Fourier, UMR 5582, BP 74, 38402 Saint-Martin-d'Hères Cedex, France • E-mail : Eric.Gaudron@ujf-grenoble.fr • Url:http://www-fourier.ujf-grenoble.fr/ gaudron

Classification mathématique par sujets (2000). — 11J86 (11J61,14G40).

Mots clefs. - Formes linéaires de logarithmes, approximation simultanée, méthode de Baker, réduction d'Hirata-Kohno, changement de variables de Chudnovsky, méthode de la section auxiliaire, fibré adélique hermitien, lemme de Siegel absolu, lemme de Siegel approché. 
valeur absolue sur le complété $\mathbf{C}_{p_{0}}$ d'une clôture algébrique de $\mathbf{Q}_{p_{0}}$ ( $p_{0}$ est un nombre premier ou $p_{0}=\infty$ avec la notation $\left.\mathbf{C}_{\infty}:=\mathbf{C}\right)$.

Depuis les travaux de Baker entrepris au milieu des années soixante, de nombreux articles ont été consacrés à l'étude de cas particuliers de ce problème. En très grande majorité les auteurs s'intéressaient à une seule forme linéaire $(t=1)$ et, souvent, seulement au cas d'un plongement archimédien. Si le plongement était ultramétrique alors ils supposaient $\beta_{i, j} \in \mathbf{Q}$. Plus précisément, dès que $t \geq 2$, et si l'on écarte les travaux de Philippon \& Waldschmidt [PW2] et d'HirataKohno [H2] qui traitent le cas très général d'un groupe algébrique commutatif quelconque, seuls Ramachandra [Ra] et Loxton [Lo] ont étudié la question qui nous intéresse ici (cas archimédien). Lorsque le plongement est ultramétrique, ne semble exister que l'étude de Dong [Do], qui traite un cas particulier de la question $\left(\beta_{i, j} \in \mathbf{Z}, \beta_{i, 0}=0\right)$. Ainsi, en dépit de la richesse de la littérature sur le thème des formes linéaires de logarithmes $(*)$, l'on ne trouve guère de résultats généraux qui prennent en compte plusieurs formes linéaires et aucun qui ne considère à la fois le cas archimédien et le cas $p$-adique. Un des objectifs de cet article est de combler cette lacune, tout en donnant une minoration qui soit en phase avec celles présentées dans [W3.

Si le résultat le plus général que nous avons ne sera donné qu'au paragraphe suivant, nous en proposons ici un cas particulier. Étant donné un nombre algébrique $\alpha$, on désigne par $h(\alpha)$ la hauteur de Weil logarithmique absolue de $\alpha$. Si $x$ est un nombre réel alors $[x]$ est la partie entière de $x$.

ThÉorème 1.1. - Soit $n \in \mathbf{N} \backslash\{0\}$. Soit $k$ un sous-corps de nombres de $\mathbf{C}$, de degré $D$ sur $\mathbf{Q}$. Soit $t \in\{1, \ldots, n\}$ et

$$
\left(\beta_{i, j}\right)_{\substack{1 \leq i \leq t \\ 1 \leq j \leq n}} \in \mathrm{M}_{t, n}(k)
$$

une matrice de rang maximal $t$. Soit $\left(\beta_{i, 0}\right)_{1 \leq i \leq t} \in k^{t}$. Pour tout $j \in\{1, \ldots, n\}$, soit $u_{j} \in \mathbf{C}$ tel que $\alpha_{j}:=e^{u_{j}} \in k$. Soit $b, a, \mathfrak{e}$ des nombres réels positifs tels que $\mathfrak{e} \geq e$,

$$
\log a \geq \max _{1 \leq j \leq n}\left\{h\left(\alpha_{j}\right), \frac{\mathfrak{e}\left|u_{j}\right|}{D}\right\}, \quad \log b \geq D \max _{\substack{1 \leq i \leq t \\ 0 \leq j \leq n}}\left\{1, h\left(\beta_{i, j}\right)\right\} .
$$

Soit a l'entier défini par

$$
\mathfrak{a}:=\left[\frac{D}{\log \mathfrak{e}} \log \left(e+\frac{D}{\log \mathfrak{e}}+\log a\right)\right]+1 .
$$

Si $\left\{u_{1}, \ldots, u_{n}\right\}$ est une famille libre sur $\mathbf{Q}$ alors les formes linéaires de logarithmes

$$
\Lambda_{i}:=\beta_{i, 0}+\beta_{i, 1} u_{1}+\cdots+\beta_{i, n} u_{n} \quad(1 \leq i \leq t)
$$

ne sont pas toutes nulles et elles vérifient la minoration

$$
\log \max _{1 \leq i \leq t}\left|\Lambda_{i}\right| \geq-(4 n)^{91 n^{2}} \mathfrak{a}^{1 / t}(\log b+\mathfrak{a} \log \mathfrak{e})\left(1+\frac{D \log a}{\log \mathfrak{e}}\right)^{n / t} .
$$

Un énoncé similaire sera aussi démontré dans le cas ultramétrique lorsque $k$ est un sous-corps de $\mathbf{C}_{p_{0}}$. Dans le minorant de (1), on notera la dépendance linéaire (et donc optimale) en $\log b$ (hauteur des formes linéaires) et la dépendance usuelle $(\log a)^{n / t}(\log \log a)^{1+1 / t}$ en $\log a$ (hauteur du point $\left.\left(\alpha_{1}, \ldots, \alpha_{n}\right)\right)$. Hormis l'aspect général de cette minoration déjà évoqué $\left(t\right.$ et $k \hookrightarrow \mathbf{C}_{p_{0}}$ quelconques), il n'y a plus le discriminant du corps de nombres, qui intervenait auparavant dans le cas simultané (comme dans l'article $\mathrm{H} 2$ d'Hirata-Kohno).

Cet article de synthèse n'apporte pas d'idée originale qui améliorerait de manière significative les résultats connus pour une forme linéaire. En revanche, la démonstration que nous proposons est, elle, plus novatrice dans sa forme car elle combine la trame de la méthode des fonctions auxiliaires classique avec les outils de la théorie des pentes adéliques (sans « méthode des pentes » proprement dite). Il en résulte un cumul des avantages propres à ces techniques : souplesse d'utilisation et simplicité de la démarche pour l'une, conservation de l'aspect intrinsèque des données et obtention aisée des constantes numériques pour l'autre. Nous expliquerons plus en détail notre approche au $\S 5.1$. Si nous avons pris le parti d'écrire ce texte dans le cas d'un groupe linéaire, il est cependant possible de généraliser à un groupe algébrique commutatif quelconque et même d'obtenir

*. Nous renvoyons le lecteur au livre très complet de Waldschmidt W3 pour en saisir l'étendue. 
des constantes numériques explicites pour une variété abélienne, comme dans [G2], sans hypothèse de non-torsion du point rationnel considéré (ici $\left.\left(\alpha_{1}, \ldots, \alpha_{n}\right) \in \mathbf{G}_{\mathrm{m}}^{n}(k)\right)$.

Remerciements. - Je remercie Daniel Bertrand et Michel Waldschmidt de leurs commentaires sur une première version de ce texte. Je remercie également Gaël Rémond pour les discussions éclairantes que nous avons eues sur les parties 3 et 4. Par ailleurs, la lecture attentive et critique du rapporteur a permis d'améliorer nombre de détails. Je lui en suis extrêmement reconnaissant. Mentionnons enfin que ce texte a bénéficié du soutien de l'ANR Diophante, 06-JCJC-0028, piloté par Lucia Di Vizio.

\section{2. Énoncé principal}

2.1. - On note $\mathcal{P}$ l'ensemble des nombres premiers auquel est adjoint un symbole supplémentaire noté $\infty$. Pour $p \neq+\infty$, on note $\left(\mathbf{C}_{p},|\cdot|_{p}\right)$ le corps valué ultramétrique complet et algébriquement clos usuel, avec la normalisation $|p|_{p}=p^{-1}$ de la valeur absolue. Si $p=\infty$, le corps valué $\left(\mathbf{C}_{\infty},|\cdot|_{\infty}\right)$ est le corps des nombres complexes $\mathbf{C}$ muni de la valeur absolue usuelle. Soit $k$ un corps de nombres de degré $D=[k: \mathbf{Q}]$. Pour chaque $p \in \mathcal{P}$ il y a $D$ morphismes de corps (appelés plongements) $\sigma: k \rightarrow \mathbf{C}_{p}$ qui correspondent aux racines dans $\mathbf{C}_{p}$ du polynôme minimal sur $\mathbf{Q}$ d'un élément primitif de $k$. Avec ces conventions, la formule du produit s'écrit

$$
\forall x \in k \backslash\{0\}, \quad \prod_{p \in \mathcal{P}} \prod_{\sigma: k \hookrightarrow \mathbf{C}_{p}}|\sigma(x)|_{p}=1
$$

et la hauteur de Weil (logarithmique absolue) d'un élément $x$ de $k$ est la somme (finie)

$$
h(x):=\frac{1}{D} \sum_{p \in \mathcal{P}} \sum_{\sigma: k \hookrightarrow \mathbf{C}_{p}} \log \max \left\{1,|\sigma(x)|_{p}\right\} .
$$

Soit $r_{p}:=p^{-1 /(p-1)}$ si $p \neq \infty$ et $r_{\infty}:=+\infty$ sinon. Désignons par $\mathcal{T}_{p}$ le disque ouvert de $\mathbf{C}_{p}$, centré en 0 et de rayon $r_{p}\left(\mathcal{T}_{\infty}=\mathbf{C}\right)$. L'exponentielle du groupe de Lie $\mathbf{G}_{\mathrm{m}}\left(\mathbf{C}_{p}\right)$ est définie sur $\mathcal{T}_{p}$ par la série convergente usuelle

$$
\forall z \in \mathcal{T}_{p}, \quad e^{z}=\sum_{i=0}^{\infty} \frac{z^{i}}{i !} .
$$

Dans la suite on fixe $p_{0} \in \mathcal{P}$ et $\sigma_{0}: k \hookrightarrow \mathbf{C}_{p_{0}}$ un plongement avec lequel nous identifierons $k$ à un sous-corps de $\mathbf{C}_{p_{0}}$. Soit $n \in \mathbf{N} \backslash\{0\}$ et $u_{1}, \ldots, u_{n}$ des éléments de $\mathcal{T}_{p_{0}}$, d'exponentielles respectives $\alpha_{1}, \ldots, \alpha_{n}$. Nous supposons que tous les $\alpha_{i}$ appartiennent à $k$.

Soit $t \in\{1, \ldots, n\}$ et $\ell_{1}, \ldots, \ell_{t}: k^{n} \rightarrow k$ des formes linéaires que l'on écrit dans la base canonique sous la forme

$$
\forall z=\left(z_{1}, \ldots, z_{n}\right) \in k^{n}, \quad \ell_{i}(z)=\beta_{i, 1} z_{1}+\cdots+\beta_{i, n} z_{n}
$$

Soit $W_{0}$ le sous-espace vectoriel de $k^{n}$ intersection des noyaux des formes linéaires $\ell_{i}, i \in\{1, \ldots, t\}$. Soit $u_{0}:=-\left(\beta_{1,0}, \ldots, \beta_{t, 0}\right) \in k^{t}$ et $u:=\left(u_{1}, \ldots, u_{n}\right) \in \mathbf{C}_{p_{0}}^{n}$. Pour tout $i \in\{1, \ldots, t\}$, posons $\Lambda_{i}:=\beta_{i, 0}+\ell_{i}(u)$. Soit $\epsilon_{0}:=0$ si $p_{0}$ est premier et $\epsilon_{0}:=1$ si $p_{0}=\infty$.

THÉORÈme 2.1. - Avec les données ci-dessus, notons $\mathrm{T}_{u}$ le sous-espace vectoriel de dimension minimale de $\mathbf{Q}^{n}$ tel que $u \in \mathbf{T}_{u} \otimes_{\mathbf{Q}} \mathbf{C}_{p_{0}}$. Considérons une partie $I$ de $\{1, \ldots, n\}$ telle que $\left(u_{i}\right)_{i \in I}$ soit une famille libre sur $\mathbf{Q}$ et maximale pour cette propriété. Nous supposons que $\left|u_{i}\right|_{p_{0}} \leq r_{p_{0}}^{2} e^{-1}$ pour tout $i \in I$. Soit $a_{1}, \ldots, a_{n}, \mathfrak{e}, \mathfrak{r}$ des nombres réels vérifiant les conditions suivantes :

$$
\begin{gathered}
\mathfrak{e} \in \begin{cases}{\left[e, \min \left\{r_{p_{0}}^{2} /\left|u_{i}\right|_{p_{0}} ; i \in I\right\}\right]} & \text { si } p_{0} \neq \infty, \\
{[e,+\infty[} & \text { si } p_{0}=\infty,\end{cases} \\
\forall j \in\{1, \ldots, n\}, \quad \log a_{j} \geq \max \left\{h\left(\alpha_{j}\right), \frac{\epsilon_{0} \mathfrak{e}\left|u_{j}\right|_{p_{0}}}{D}\right\} .
\end{gathered}
$$

Soit a l'entier défini par la formule

$$
\mathfrak{a}:=\left[\frac{D}{\log \mathfrak{e}} \log \left(e+\frac{D}{\log \mathfrak{e}}+\log \prod_{j=1}^{n} a_{j}\right)\right]+1 .
$$


Soit b un nombre réel vérifiant

$$
\begin{gathered}
\log b \geq D \max _{\substack{1 \leq j \leq t \\
0 \leq \ell \leq n}}\left\{1, h\left(\beta_{j, \ell}\right)\right\} \\
\text { et } s:=\operatorname{dim} \mathrm{T}_{u}-\operatorname{dim}\left(W_{0} \cap \mathrm{T}_{u}\right) \text {. Alors, s'il existe } j \in\{1, \ldots, t\} \text { tel que } \Lambda_{j} \neq 0 \text {, on } a \\
\log \max _{1 \leq j \leq t}\left|\Lambda_{j}\right|_{p_{0}} \geq-(4 n)^{91 n^{2}} \mathfrak{a}^{1 / s}(\log b+\mathfrak{a} \log \mathfrak{e}+D \log \log \mathfrak{e}) \prod_{i \in I}\left(1+\frac{D \log a_{i}}{\log \mathfrak{e}}\right)^{1 / s} .
\end{gathered}
$$

Le théorème de Wüstholz Wü] affirme que $\mathrm{T}_{u}$ est l'espace tangent d'un sous-groupe algébrique $G_{u}$ de $\mathbf{G}_{\mathrm{m}}^{n}$. Le paramètre $s$ est donc identique à celui de $[\mathrm{DH}$, article dans lequel il apparaît pour la première fois. On notera au passage que $\operatorname{dim} \mathrm{T}_{u}=$ card $I$. Par ailleurs, nous verrons au $\S 5.2$ que si $s=1$, la quantité $\log b+\mathfrak{a} \log \mathfrak{e}+D \log \log \mathfrak{e}$ peut être remplacée par $\log (b \mathfrak{e})+D \log \mathfrak{a}+D \log \log \mathfrak{e}$.

\subsection{Commentaires. -}

1) La différence avec le résultat de Loxton [Lo est assez modeste. Elle réside surtout dans la dépendance en le degré $D$ (ici de l'ordre de $D^{1+\varepsilon+(n+1) / s}$ au lieu de $D^{200 n}$ ) et dans le caractère plus général du théorème 2.1 (plongement $\sigma_{0}$ quelconque, pas d'hypothèse d'indépendance multiplicative des $\left.\alpha_{j}, 1 \leq j \leq n\right)$. En revanche Loxton a une constante égale à $(16 n)^{200 n}$.

2) Lorsque $p_{0}$ est premier, je n'ai pas trouvé dans la littérature de minoration analogue à celle du théorème 2.1] bien que la méthode de Baker originelle puisse donner un tel résultat. Il semble que l'obtention de minorations générales et explicites ait été délaissée au profit de l'étude de cas particuliers comme le cas rationnel $\left(\beta_{i, j} \in \mathbf{Z}, \beta_{i, 0}=0\right)$, a priori plus utiles pour les applications (voir le survol [Yu] et [W3, p. 547]).

3) Toujours dans le cas $p$-adique, on observe que la minoration de $\log \max _{i}\left|\Lambda_{i}\right|_{p_{0}}$ ne dépend pas de $p_{0}$. En réalité cette dépendance est cachée dans la condition $\left|u_{i}\right|_{p_{0}} \leq r_{p_{0}}^{2} e^{-1}$ qui est assez forte. Nous aurions pu affaiblir la condition en $\left|u_{i}\right|_{p_{0}}<r_{p_{0}}^{2}$ mais au prix d'une complexité accrue du minorant de $\log \max _{i}\left|\Lambda_{i}\right|_{p_{0}}$, à l'instar du théorème de Rémond \& Urfels [RU] (qui est dans un contexte un peu différent). Nous avons donc opté pour un compromis entre une hypothèse un peu plus forte et un résultat un peu plus lisible et maniable. Dans le cas rationnel, Yu parvient à des hypothèses très faibles du type $\left|u_{i}\right|_{p_{0}} \leq 1$.

4) La constante $(4 n)^{91 n^{2}}$ qui apparaît dans le théorème 2.1 a une moins bonne dépendance en $n$ que celle de Loxton car nous n'avons pas mis en œuvre la méthode de Kummer. Rappelons que dans le cas rationnel, avec $t=1$ et $p_{0}=\infty$, Matveev a montré que l'on pouvait avoir une constante du type $c^{n}$ avec $c$ absolue (voir $\mathrm{Ma}$ ). Il serait intéressant d'incorporer sa méthode dans le cadre de la théorie des pentes que nous utilisons ici. Quant à l'aspect numérique de la constante $(4 n)^{91 n^{2}}$, une grand partie de sa taille vient du fait qu'il n'a pas été possible de supposer $n \geq 2$ dans la démonstration (à cause du cas $p$-adique, voir plus haut). Une meilleure constante serait facilement accessible si l'on traitait séparément les cas $n=1$ et $n \geq 2$ par exemple.

La démonstration du théorème 2.1 commence au $\S$ [5 avec une réduction au cas $\mathrm{T}_{u}=\mathbf{Q}^{n}$ (la famille $\left\{u_{1}, \ldots, u_{n}\right\}$ est libre sur $\left.\mathbf{Q}\right)$. Nous expliquerons son déroulement à cette occasion ( $\$$ 5.1) mais, auparavant, nous rappelons quelques notions de théorie des pentes adéliques et nous énonçons un nouveau lemme de Siegel approché qui jouera un rôle clef dans la démonstration.

\section{3. Éléments de théorie des pentes adéliques}

La théorie des pentes des fibrés vectoriels adéliques sur un corps de nombres $k$ a fait l'objet d'une présentation systématique dans l'article G4]. Nous adoptons ici une présentation un peu décalée où les places du corps de nombres sont remplacées par les plongements dans les corps $\mathbf{C}_{p}$ pour $p$ un nombre premier ou $p=\infty$ (dans ce dernier cas $\mathbf{C}_{\infty}$ désigne le corps des nombres complexes C). Rappelons que $\mathcal{P}$ désigne l'ensemble des nombres premiers et de l'infini, $\mathcal{P}:=\{2,3,5, \ldots, \infty\}$. 
3.1. Définitions. - Un fibré vectoriel pré-adélique $\bar{E}=\left(E,\left(\|\cdot\|_{\bar{E}, \sigma}\right)_{\sigma}\right)$ est la donnée d'un $k$ espace vectoriel $E$, de dimension finie $\nu \geq 0$, et, pour tout $p \in \mathcal{P}$ et tout plongement $\sigma: k \hookrightarrow \mathbf{C}_{p}$, d'une norme $\|\cdot\|_{\bar{E}, \sigma}$ sur $E \otimes_{\sigma} \mathbf{C}_{p}$, qui satisfont aux contraintes suivantes :

1) il existe une $k$-base $\left(e_{1}, \ldots, e_{\nu}\right)$ de $E$ telle que, pour tout $p \in \mathcal{P}$ sauf un nombre fini, pour tout plongement $\sigma: k \hookrightarrow \mathbf{C}_{p}$, on a $\left\|\sum_{i=1}^{\nu} e_{i} \otimes_{\sigma} x_{i}\right\|_{E, \sigma}=\max \left\{\left|x_{1}\right|_{p}, \ldots,\left|x_{\nu}\right|_{p}\right\}$ pour tout $\left(x_{1}, \ldots, x_{\nu}\right) \in$ $\mathbf{C}_{p}^{\nu}$

2) si $p \neq \infty$ alors $\|\cdot\|_{\bar{E}, \sigma}$ est une ultranorme.

Dans cette définition, la notation $E \otimes_{\sigma} \mathbf{C}_{p}$ désigne le produit tensoriel $E \otimes_{k} \mathbf{C}_{p}$ où $\mathbf{C}_{p}$ est muni de la structure de $k$-espace vectoriel donnée par $\lambda . x:=\sigma(\lambda) x$ pour $\lambda \in k$ et $x \in \mathbf{C}_{p}$. Dans la suite, l'espace $E \otimes_{\sigma} \mathbf{C}_{p}$ est vu comme espace vectoriel sur $\mathbf{C}_{p}$ avec la loi externe $\mu_{1} \cdot\left(x \otimes \mu_{2}\right):=x \otimes\left(\mu_{1} \mu_{2}\right)$ pour $x \in E$ et $\mu_{1}, \mu_{2} \in \mathbf{C}_{p}$. En particulier, pour $\lambda \in k$ et $x \in E \otimes_{\sigma} \mathbf{C}_{p}$, on a $\|\lambda . x\|_{\bar{E}, \sigma}=|\sigma(\lambda)|_{p}\|x\|_{E, \sigma}$. On rappelle que deux plongements $\sigma, \sigma^{\prime}$ de $k$ dans $\mathbf{C}_{p}$ sont dits conjugués s'il existe un automorphisme continu $\iota: \mathbf{C}_{p} \rightarrow \mathbf{C}_{p}$ tel que $\sigma^{\prime}=\iota \circ \sigma$. Ceci définit une relation d'équivalence et une place de $k$ est une classe d'équivalence de plongements.

Définition 3.1. - Soit $p \in \mathcal{P}$ et $v$ une place de $k$ au-dessus de $p$. On dit que la collection de normes $\left(\|\cdot\|_{\bar{E}, \sigma}\right)_{\sigma \in v}$ est invariante par Galois si, pour tout automorphisme continu $\iota: \mathbf{C}_{p} \rightarrow \mathbf{C}_{p}$, l'application canonique

$$
\operatorname{id} \otimes \iota:\left(E \otimes_{\sigma} \mathbf{C}_{p},\|\cdot\|_{E, \sigma}\right) \rightarrow\left(E \otimes_{\iota \circ \sigma} \mathbf{C}_{p},\|\cdot\|_{\bar{E}, \iota \circ \sigma}\right)
$$

est une isométrie.

Quand la collection de normes $\left(\|\cdot\|_{\bar{E}, \sigma}\right)_{\sigma \in v}$ est invariante par Galois, elle ne dépend que de la place $v$ de $k$ induite par $\sigma$ et il est naturel alors de la noter $\|\cdot\|_{\bar{E}, v}$. Par exemple, si $E=k^{\nu}$ et si $\sigma: k \hookrightarrow \mathbf{C}$ est un plongement complexe, la paire $\left\{\|\cdot\|_{\bar{E}, \sigma},\|\cdot\|_{\bar{E}, \bar{\sigma}}\right\}$ (ou, par abus de langage, la norme $\left.\|\cdot\|_{\bar{E}, \sigma}\right)$ est invariante par Galois si $\left\|\left(x_{1}, \ldots, x_{\nu}\right)\right\|_{\bar{E}, \sigma}=\left\|\left(\overline{x_{1}}, \ldots, \overline{x_{\nu}}\right)\right\|_{\bar{E}, \bar{\sigma}}$ pour tout $\left(x_{1}, \ldots, x_{\nu}\right) \in \mathbf{C}^{\nu}$, car il n'y a que deux automorphismes continus de $\mathbf{C}$ qui sont l'identité et la conjugaison complexe. Si, de plus, $\sigma$ est réel $(\sigma(k) \subseteq \mathbf{R})$ et si $\|\cdot\|_{\bar{E}, \sigma}$ est donné par une matrice hermitienne $A \in \mathrm{M}_{\nu}(\mathbf{C})$ définie positive (c'est-à-dire $\|x\|_{\bar{E}, \sigma}=\left({ }^{\mathrm{t}} \bar{x} A x\right)^{1 / 2}$ pour tout $x \in \mathbf{C}^{\nu}$ ) alors nécessairement $A$ est une matrice symétrique réelle.

DÉfinition 3.2. - Un fibré vectoriel adélique sur $k$ est un fibré vectoriel pré-adélique $\bar{E}=(E,(\|$. $\left.\left.\|_{\bar{E}, \sigma}\right)_{\sigma}\right)$ sur $k$ tel que, pour toute place $v$ de $k$, la collection de normes $\left(\|\cdot\|_{\bar{E}, \sigma}\right)_{\sigma \in v}$ est invariante par Galois.

On le note alors habituellement $\bar{E}=\left(E,\left(\|\cdot\|_{\bar{E}, v}\right)_{v}\right)$ où $v$ parcourt les places de $k$. C'est le point de vue adopté dans G4.

Soit $\bar{E}$ un fibré vectoriel pré-adélique. Si $F$ est un sous-espace vectoriel de $E$, il induit un sousfibré vectoriel pré-adélique $\bar{F}$, dont les normes sont les restrictions de celles de $\bar{E}$ à $F$. Le fibré vectoriel pré-adélique est dit hermitien lorsque toutes les normes correspondant aux plongements complexes $\sigma: k \hookrightarrow \mathbf{C}$ sont hermitiennes. Il est dit pur ${ }^{(\dagger)}$ lorsque, pour tout plongement ultramétrique $\sigma: k \hookrightarrow \mathbf{C}_{p}$ et tout $x \in E$, on a $\|x\|_{E, \sigma} \in\left|k_{\sigma}\right|_{p}$ où $k_{\sigma}$ est l'adhérence topologique de $\sigma(k)$ dans $\mathbf{C}_{p}$ (voir [G5]). Contrairement à la définition 2.3 de [G5, il n'est pas supposé ici qu'un fibré pré-adélique hermitien est nécessairement pur. Les fibrés adéliques hermitiens purs sont exactement les fibrés vectoriels hermitiens sur $\operatorname{Spec} \mathcal{O}_{k}$ de la géométrie d'Arakelov classique (voir proposition 3.10). L'exemple le plus simple d'un tel fibré est celui du fibré standard $\left(k^{\nu},|\cdot|_{2}\right)$ que l'on obtient de la manière suivante. Étant donné $p \in \mathcal{P}$ et $x_{1}, \ldots, x_{\nu}$ des nombres réels, notons

$$
\operatorname{moy}_{p}\left(x_{1}, \ldots, x_{\nu}\right):= \begin{cases}\left(\sum_{i=1}^{\nu} x_{i}^{2}\right)^{1 / 2} & \text { si } p=\infty, \\ \max \left\{x_{1}, \ldots, x_{\nu}\right\} & \text { si } p \neq \infty .\end{cases}
$$

Le fibré vectoriel adélique $\left(k^{\nu},|\cdot|_{2}\right)$, hermitien et pur, est par définition le fibré adélique d'espace sous-jacent $k^{\nu}$ et de normes $|x|_{2, \sigma}:=\operatorname{moy}_{p}\left(\left|x_{1}\right|_{p}, \ldots,\left|x_{\nu}\right|_{p}\right)$ pour tout $x=\left(x_{1}, \ldots, x_{\nu}\right) \in \mathbf{C}_{p}^{\nu}$. Après le choix d'une $k$-base (quelconque) $\left(e_{1}, \ldots, e_{\nu}\right)$ de $E$, les fibrés vectoriels hermitiens sur

†. La notion de pureté d'une norme est un cas particulier de la propriété (N) de Serre [Se p. 69]. La terminologie solid norm est également parfois employée dans d'autres contextes [PGS] p. 19]. 
Spec $\mathcal{O}_{k}$ s'obtiennent à partir du fibré standard $\left(k^{\nu},|\cdot|_{2}\right)$ au moyen d'une collection de matrices $\left\{A_{\sigma} \in \mathrm{GL}_{\nu}\left(k_{\sigma}\right) ; \sigma: k \hookrightarrow \mathbf{C}_{p}\right\}_{p \in \mathcal{P}}$ telles que

1) pour tout $\sigma$, sauf un nombre fini, $A_{\sigma}$ est une isométrie de $\left(\mathbf{C}_{p}^{\nu},|\cdot|_{2, \sigma}\right)$,

2) pour tout automorphisme continu $\iota: \mathbf{C}_{p} \rightarrow \mathbf{C}_{p}$, on a $A_{\iota \circ \sigma}=\iota\left(A_{\sigma}\right)$ (l'action de $\iota$ est sur les coefficients de la matrice),

$3)$ pour tous $x_{1}, \ldots, x_{\nu} \in \mathbf{C}_{p}$ on a

$$
\left\|e_{1} \otimes_{\sigma} x_{1}+\cdots+e_{\nu} \otimes_{\sigma} x_{\nu}\right\|_{\bar{E}, \sigma}=\left|A_{\sigma}\left(\begin{array}{c}
x_{1} \\
\vdots \\
x_{\nu}
\end{array}\right)\right|_{2, \sigma} .
$$

3.2. Extension des scalaires. - Soit $\bar{E}$ un fibré vectoriel pré-adélique sur $k$ et $K$ une extension finie de $k$ (on identifie $k$ à un sous-corps de $K$ ). Le $K$-espace vectoriel $E_{K}:=E \otimes_{k} K$ est muni naturellement d'une structure de fibré vectoriel pré-adélique $\overline{E_{K}}$ de la manière suivante. Soit $\tau$ : $K \hookrightarrow \mathbf{C}_{p}$ un plongement qui étend $\sigma: k \hookrightarrow \mathbf{C}_{p}$. Pour des éléments $e_{i} \in E, \lambda_{i} \in K, x_{i} \in \mathbf{C}_{p}$ posons

$$
\left\|\sum_{i}\left(e_{i} \otimes_{k} \lambda_{i}\right) \otimes_{\tau} x_{i}\right\|_{E_{K}, \tau}:=\left\|\sum_{i} e_{i} \otimes_{\sigma}\left(\tau\left(\lambda_{i}\right) x_{i}\right)\right\|_{\bar{E}, \sigma} .
$$

En choisissant des bases de $E$ sur $k$ et de $K \operatorname{sur} k$, on vérifie que cette définition a bien un sens et que la norme d'un élément $x \in\left(E \otimes_{k} K\right) \otimes_{\tau} \mathbf{C}_{p}$ ne dépend pas du choix de sa représentation en somme de tenseurs élémentaires. Le couple $\overline{E_{K}}=\left(E \otimes_{k} K,\left(\|\cdot\|_{E_{K}}, \tau\right)\right.$ forme alors un fibré vectoriel pré-adélique sur $K$. Il est adélique si $\bar{E}$ l'est car, si $\iota: \mathbf{C}_{p} \rightarrow \mathbf{C}_{p}$ est un automorphisme continu de corps, on a

$$
\begin{aligned}
\left\|\sum_{i}\left(e_{i} \otimes_{k} \lambda_{i}\right) \otimes_{\iota \circ \tau} \iota\left(x_{i}\right)\right\|_{\overline{E_{K}}, \iota \circ \tau} & =\left\|\sum_{i} e_{i} \otimes_{\iota \circ \sigma}\left((\iota \circ \tau)\left(\lambda_{i}\right) \iota\left(x_{i}\right)\right)\right\|_{\bar{E}, \iota \circ \sigma} \text { par définition } \\
& =\left\|\sum_{i} e_{i} \otimes_{\iota \circ \sigma} \iota\left(\tau\left(\lambda_{i}\right) x_{i}\right)\right\|_{\bar{E}, \iota \circ \sigma} \\
& =\left\|\sum_{i} e_{i} \otimes_{\sigma}\left(\tau\left(\lambda_{i}\right) x_{i}\right)\right\|_{\bar{E}, \sigma} \text { par isométrie } \\
& =\left\|\sum_{i}\left(e_{i} \otimes_{k} \lambda_{i}\right) \otimes_{\tau} x_{i}\right\|_{\overline{E_{K}}, \tau} \text { par définition. }
\end{aligned}
$$

De même, si la norme $\|\cdot\|_{\bar{E}, \sigma}$ est hermitienne alors $\|\cdot\|_{\overline{E_{K}}, \tau}$ l'est aussi pour tout plongement $\tau$ qui étend $\sigma$.

3.3. Produit tensoriel de fibrés hermitiens. - Soit $\bar{E}$ et $\bar{F}$ des fibrés pré-adéliques hermitiens sur $k$. On munit $E \otimes_{k} F$ d'une structure de fibré pré-adélique hermitien de la manière suivante. Si $\sigma: k \hookrightarrow \mathbf{C}$ est un plongement complexe, on considère des bases orthonormées $\mathrm{e}_{1}, \ldots, \mathrm{e}_{\nu}$ de $\left(E \otimes_{\sigma} \mathbf{C},\|\cdot\|_{\bar{E}, \sigma}\right)$ et $\mathrm{f}_{1}, \ldots, \mathrm{f}_{\mu}$ de $\left(F \otimes_{\sigma} \mathbf{C},\|\cdot\|_{\bar{F}, \sigma}\right)$ et on choisit sur $\left(E \otimes_{k} F\right) \otimes_{\sigma} \mathbf{C} \simeq$ $\left(E \otimes_{\sigma} \mathbf{C}\right) \otimes_{\mathbf{C}}\left(F \otimes_{\sigma} \mathbf{C}\right)$ l'unique produit hermitien pour lequel $\left(\mathrm{e}_{i} \otimes \mathrm{f}_{j}\right)_{i, j}$ forme une base orthonormée (indépendant du choix des bases). Si $\sigma: k \hookrightarrow \mathbf{C}_{p}$ est un plongement ultramétrique, on identifie $E \otimes_{k} F$ à $\operatorname{Hom}_{k}\left(E^{\vee}, F\right)$ où $E^{\vee}=\operatorname{Hom}_{k}(E, k)$ désigne le dual de $E$ et l'on munit $\left(E \otimes_{k} F\right) \otimes_{\sigma} \mathbf{C}_{p}$ de la norme d'opérateur sur $\operatorname{Hom}_{k}\left(E^{\mathrm{v}}, F\right) \otimes_{\sigma} \mathbf{C}_{p}$. Autrement dit, si $x=\sum_{i=1}^{N} x_{i} \otimes y_{i}$ avec $x_{i} \in E \otimes_{\sigma} \mathbf{C}_{p}$ et $y_{i} \in F \otimes_{\sigma} \mathbf{C}_{p}$, on a

$$
\|x\|_{\overline{E \otimes F}, \sigma}:=\sup \left\{\frac{\left\|\sum_{i=1}^{N} \varphi\left(x_{i}\right) y_{i}\right\|_{\bar{F}, \sigma}}{\|\varphi\|_{\overline{E^{v}}, \sigma}} ; \varphi \in\left(E^{\vee} \otimes_{\sigma} \mathbf{C}_{p}\right) \backslash\{0\}\right\} .
$$

On vérifie que le couple $\bar{E} \otimes \bar{F}=\left(E \otimes_{k} F,\left(\|\cdot\|_{\overline{E \otimes F}, \sigma}\right)_{\sigma}\right)$ forme un fibré pré-adélique hermitien. De plus si $\bar{E}$ et $\bar{F}$ sont des fibrés adéliques hermitiens (resp. purs), il en est de même pour $\bar{E} \otimes \bar{F}$. Lorsque $\bar{E}$ est un fibré pré-adélique hermitien, de dimension $\nu \geq 1$, et $\mu \in\{1, \ldots, \nu\}$, on peut alors munir la puissance extérieure $\wedge^{\mu} E$ de $E$ de normes lui conférant une structure de fibré pré-adélique $\overline{\wedge^{\mu} E}$ 
sur $k$ de la manière suivante. Si $\sigma$ est un plongement complexe, on considère une base orthonormée $\mathrm{e}_{1}, \ldots, \mathrm{e}_{\nu}$ de $\left(E \otimes_{\sigma} \mathbf{C},\|\cdot\|_{\bar{E}, \sigma}\right)$ et on décrète que la base $\left\{\mathrm{e}_{i_{1}} \wedge \cdots \wedge \mathrm{e}_{i_{\mu}} ; 1 \leq i_{1}<\cdots<i_{\mu} \leq \nu\right\}$ de $\wedge^{\mu} E \otimes_{\sigma} \mathbf{C}$ est orthonormée. Si $\sigma$ est un plongement ultramétrique, on voit $\wedge^{\mu} E \otimes_{\sigma} \mathbf{C}_{p}$ comme un quotient de $\left(E \otimes_{\sigma} \mathbf{C}_{p}\right)^{\otimes \mu}$ que l'on munit de la norme quotient induite par $\|\cdot\|_{E^{\otimes \mu}, \sigma}$.

3.4. Pentes des fibrés pré-adéliques hermitiens. - On note det $E$ la puissance extérieure maximale $\wedge^{\nu} E$ de $E$.

DÉFInition 3.3. - Le degré d'Arakelov (normalisé) du fibré pré-adélique hermitien E est le nombre réel

$$
\widehat{\operatorname{deg}}_{\mathrm{n}} \bar{E}:=-\frac{1}{D} \sum_{p \in \mathcal{P}} \sum_{\sigma: k \hookrightarrow \mathbf{C}_{p}} \log \left\|e_{1} \wedge \cdots \wedge e_{\nu}\right\|_{\overline{\operatorname{det} E}, \sigma}
$$

pour tout choix d'une $k$-base $\left(e_{1}, \ldots, e_{\nu}\right)$ de $E$. Lorsque $E=\{0\}$ on pose $\widehat{\operatorname{deg}}_{\mathrm{n}} \bar{E}:=0$.

L'indépendance du degré par rapport au choix de cette base est une conséquence de la formule du produit. La hauteur (logarithmique absolue) de $\bar{E}$ est $h(\bar{E}):=-\widehat{\operatorname{deg}}_{\mathrm{n}} \bar{E}$, sa pente d'Arakelov normalisée (si $E \neq\{0\}$ ) est

sa pente maximale (si $E \neq\{0\}$ ) est

$$
\widehat{\mu}(\bar{E}):=\frac{\widehat{\operatorname{deg}}_{\mathrm{n}} E}{\operatorname{dim} E}
$$

$$
\widehat{\mu}_{\max }(\bar{E}):=\max \{\widehat{\mu}(\bar{F}) ;\{0\} \neq F \subseteq E\} .
$$

On a également une notion de hauteur pour les éléments $x$ de $E$ :

$$
\forall x \in E \backslash\{0\}, \quad h_{\bar{E}}(x):=\frac{1}{D} \sum_{p \in \mathcal{P}} \sum_{\sigma: k \hookrightarrow \mathbf{C}_{p}} \log \|x\|_{\bar{E}, \sigma} \quad\left(h_{\bar{E}}(0):=-\infty\right) .
$$

LEMME 3.4. - Soit $\bar{E}$ un fibré pré-adélique hermitien sur $k$.

1) Si $\bar{F}$ est un sous-fibré de $\bar{E}$ alors on a $\widehat{\operatorname{deg}}_{\mathrm{n}} \bar{E} \bar{E}=\widehat{\operatorname{deg}}_{\mathrm{n}} \bar{E}-\widehat{\operatorname{deg}}_{\mathrm{n}} \bar{F}$.

2) $S i \overline{E_{1}}, \overline{E_{2}}$ sont des sous-fibrés de $\bar{E}$ alors on a

$$
\widehat{\operatorname{deg}}_{n} \overline{E_{1}}+\widehat{\operatorname{deg}}_{n} \overline{E_{2}} \leq \widehat{\operatorname{deg}}_{n} \overline{E_{1}+E_{2}}+\widehat{\operatorname{deg}}_{n} \overline{E_{1} \cap E_{2}} .
$$

Démonstration. - La démonstration est identique à celle des fibrés vectoriels hermitiens sur $\operatorname{Spec} \mathcal{O}_{k}$ (voir [G4, propositions 4.22 et 4.23$]$ ).

Toutes ces notions sont invariantes par extension des scalaires :

Proposition 3.5. - Soit $\bar{E}$ un fibré pré-adélique hermitien sur $k$ et $K / k$ une extension finie. Alors on $a \widehat{\operatorname{deg}}_{\mathrm{n}} \overline{E_{K}}=\widehat{\operatorname{deg}}_{\mathrm{n}} \bar{E}$ et $\widehat{\mu}_{\max }\left(\overline{E_{K}}\right)=\widehat{\mu}_{\max }(\bar{E})$.

Démonstration. - Le nombre de plongements $\tau: K \hookrightarrow \mathbf{C}_{p}$ qui prolongent $\sigma: k \hookrightarrow \mathbf{C}_{p}$ est égal à $[K: k]$, ce qui donne la première propriété. Pour la seconde, on montre comme dans le cas classique l'existence et l'unicité du fibré déstabilisant (en utilisant le lemme précédent), c'est-à-dire qu'il existe un unique sous-fibré $\overline{D_{k}}$ de $\bar{E}$, de dimension maximale, tel que $\widehat{\mu}_{\max }(\bar{E})=\widehat{\mu}\left(\overline{D_{k}}\right)$. Comme $D_{k} \otimes_{k} K$ est un sous-espace de $E_{K}$, on a

$$
\widehat{\mu}_{\max }(\bar{E})=\widehat{\mu}\left(\overline{D_{k}}\right)=\widehat{\mu}\left(\left(\overline{D_{k}}\right)_{K}\right) \leq \widehat{\mu}_{\max }\left(\overline{E_{K}}\right) .
$$

Pour démontrer l'égalité, on peut donc supposer que $K / k$ est galoisienne. Considérons le morphisme de groupes $\rho: \operatorname{Gal}(K / k) \rightarrow \mathrm{GL}\left(E_{K}\right)$ qui à $u \in \operatorname{Gal}(K / k)$ associe l'isomorphisme $\rho(u)=\operatorname{id}_{E} \otimes u$ de $E_{K}=E \otimes_{k} K$. Pour tout $m \in\{1, \ldots, \operatorname{dim} E\}$, pour tous $e_{1}, \ldots, e_{m} \in E_{K}$, pour tout plongement $\tau: K \hookrightarrow \mathbf{C}_{p}$, on a

$$
\left\|\rho(u)\left(e_{1}\right) \wedge \cdots \wedge \rho(u)\left(e_{m}\right)\right\|_{\overline{\wedge^{m} E}, \tau}=\left\|e_{1} \wedge \cdots \wedge e_{m}\right\|_{\overline{\wedge^{m} E}, \tau \circ u} .
$$

En appliquant cette égalité à une $K$-base de $D_{K}$, on obtient $\widehat{\mu}\left(\overline{\rho(u)\left(D_{K}\right)}\right)=\widehat{\mu}\left(\overline{D_{K}}\right)$. Par unicité de $\overline{D_{K}}$ on a $\rho(u)\left(D_{K}\right)=D_{K}$ pour tout $u \in \operatorname{Gal}(K / k)$. Il existe alors un sous-espace vectoriel $D_{0}$ de $E$ tel que $D_{K}=D_{0} \otimes_{k} K$. En effet, si, pour $x \in E_{K}$, on pose $\operatorname{tr}(x):=\sum_{u \in \operatorname{Gal}(K / k)} \rho(u)(x) \in E$, alors $D_{0}=\left\{\operatorname{tr}(x) ; x \in D_{K}\right\}$ convient. On conclut avec $\widehat{\mu}_{\max }\left(\overline{E_{K}}\right)=\widehat{\mu}\left(\overline{D_{K}}\right)=\widehat{\mu}\left(\overline{D_{0}}\right) \leq \widehat{\mu}_{\max }(\bar{E})$. 
L'argument sur le nombre de plongements $\tau: K \hookrightarrow \mathbf{C}_{p}$ qui prolongent $\sigma: k \hookrightarrow \mathbf{C}_{p}$ permet de voir également que le nombre réel $h_{\overline{E_{K}}}(x)$ ne dépend pas du choix de l'extension $K / k$ pour laquelle $x \in E \otimes_{k} K$ (lorsque $\bar{E}$ est un fibré vectoriel pré-adélique).

L'énoncé suivant remplace l'inégalité de Liouville usuelle.

Lemme 3.6. - Soit $\bar{E}$ un fibré pré-adélique hermitien non nul. Alors

$$
\forall x \in E \backslash\{0\}, \quad h_{\bar{E}}(x) \geq-\widehat{\mu}_{\max }(\bar{E}) .
$$

La démonstration est immédiate à partir des définitions car $h_{\bar{E}}(x)=h\left(\left(k \cdot x,\left(\|\cdot\|_{\bar{E}, \sigma}\right)_{\sigma}\right)\right)$.

3.5. Compléments sur les fibrés adéliques. - Soit $(K,|\cdot|)$ un corps valué ultramétrique complet. Soit $\mathrm{E}$ un $\mathrm{K}$-espace vectoriel de dimension $\nu \geq 1$ et $\|\cdot\|$ une ultranorme sur $\mathrm{E}$.

DÉfinition 3.7. - Soit $a \in] 0,1]$. On dit qu'une famille $\left\{\mathrm{e}_{1}, \ldots, \mathrm{e}_{\nu}\right\}$ de vecteurs de $\mathrm{E}$ est $a$ orthogonale si, pour tous $x_{1}, \ldots, x_{\nu} \in \mathrm{K}$, on a

$$
a \max _{1 \leq i \leq \nu}\left|x_{i}\right|\left\|\mathrm{e}_{i}\right\| \leq\left\|x_{1} \mathrm{e}_{1}+\cdots+x_{\nu} \mathrm{e}_{\nu}\right\| .
$$

Si $a=1$ on dit que la base est orthogonale et si, de plus, $\left\|\mathrm{e}_{1}\right\|=\cdots=\left\|\mathrm{e}_{\nu}\right\|=1$ alors la base est dite orthonormée.

Lorsque $a<1$, de telles bases existent par le critère suivant dû à Van der Put (voir PGS, théorème 2.2.16]). Si e $\in \mathrm{E}$ et $\mathrm{F} \subseteq \mathrm{E}$ est un sous-espace vectoriel, on note $\operatorname{dist}(\mathrm{e}, \mathrm{F})$ la borne inférieure des $\|e-f\|$ pour $f \in F$.

Lemme 3.8. - Soit $\left.\left.t_{2}, \ldots, t_{\nu} \in\right] 0,1\right]$ et $\left(\mathrm{e}_{1}, \ldots, \mathrm{e}_{\nu}\right)$ une base de $\mathrm{E}$ tels que, pour tout $i \in\{2, \ldots, \nu\}$, on $a$

$$
\operatorname{dist}\left(\mathrm{e}_{i}, \mathrm{~K}_{\mathrm{e}} \mathrm{e}_{1}+\cdots+\mathrm{K}_{\mathrm{e}} \mathrm{e}_{i-1}\right) \geq t_{i}\left\|\mathrm{e}_{i}\right\| .
$$

Alors $\left(\mathrm{e}_{1}, \ldots, \mathrm{e}_{\nu}\right)$ est une base $t_{2} \cdots t_{\nu}$-orthogonale de $\mathrm{E}$. En particulier, pour tout $\left.a \in\right] 0,1[$, il existe une base a-orthogonale de $\mathrm{E}$.

Dans le cas d'une base orthogonale, la réciproque du lemme 3.8 est vraie : si $\left(\mathrm{e}_{1}, \ldots, \mathrm{e}_{\nu}\right)$ est une base orthogonale de $(\mathrm{E},\|\cdot\|)$ alors, pour tout $i \in\{2, \ldots, \nu\}$, on a $\operatorname{dist}\left(\mathrm{e}_{i}, \mathrm{~K} \cdot \mathrm{e}_{1}+\cdots+\mathrm{K} \cdot \mathrm{e}_{i-1}\right)=\left\|\mathrm{e}_{i}\right\|$.

DÉFInition 3.9. - L'espace normé $(\mathrm{E},\|\cdot\|)$ est dit sphériquement complet si toute suite de boules emboîtées de $\mathrm{E}$ a une intersection non vide.

Un corps local (†)], par exemple $\mathbf{Q}_{p}$, est sphériquement complet. On montre que $\mathbf{E}$ (de dimension finie) est sphériquement complet si et seulement si $(\mathrm{K},|\cdot|)$ l'est. Dans ce cas, la distance dist (e, F) est un minimum et, en particulier, $(E,\|\cdot\|)$ possède une base orthogonale (cette propriété est en général fausse si $\mathrm{K}$ n'est pas sphériquement complet, comme par exemple $\mathrm{K}=\mathbf{C}_{p}$, voir [PGS, exemple $2.3 .26])$.

Lemme De Perturbation. - Soit E un espace vectoriel de dimension finie sur un corps valué ultramétrique $(\mathrm{K},|\cdot|)$ et $\|\cdot\|$ une ultranorme sur $\mathrm{E}$. Soit a $\in] 0,1]$ et $\left(\mathrm{e}_{1}, \ldots, \mathrm{e}_{\nu}\right)$ une base aorthogonale de $\mathrm{E}$. Soit $\mathrm{f}_{1}, \ldots, \mathrm{f}_{\nu} \in \mathrm{E}$ tels que $\left\|\mathrm{e}_{i}-\mathrm{f}_{i}\right\|<a\left\|\mathrm{e}_{i}\right\|$ pour tout $i \in\{1, \ldots, \nu\}$. Alors $\left(\mathrm{f}_{1}, \ldots, \mathrm{f}_{\nu}\right)$ est une base a-orthogonale de $\mathrm{E}$.

Démonstration. - Par inégalité ultramétrique on a $\left\|\mathrm{f}_{i}\right\|=\left\|\mathrm{e}_{i}\right\|$. On en déduit, pour tous $x_{1}, \ldots, x_{\nu} \in$ $\mathrm{K}$,

$$
\begin{aligned}
a \max _{1 \leq i \leq \nu}\left|x_{i}\right|\left\|\mathrm{f}_{i}\right\| & =a \max _{1 \leq i \leq \nu}\left|x_{i}\right|\left\|\mathrm{e}_{i}\right\| \leq\left\|\sum_{i=1}^{\nu} x_{i} \mathrm{e}_{i}\right\| \\
& \leq \max \left(\left\|\sum_{i=1}^{\nu} x_{i} \mathrm{f}_{i}\right\|,\left\|\sum_{i=1}^{\nu} x_{i}\left(\mathrm{f}_{i}-\mathrm{e}_{i}\right)\right\|\right) .
\end{aligned}
$$

Si au moins un des $x_{i}$ n'est pas nul on a $\left\|\sum_{i=1}^{\nu} x_{i}\left(\mathrm{f}_{i}-\mathrm{e}_{i}\right)\right\|<a \max _{1 \leq i \leq \nu}\left|x_{i}\right|\left\|\mathrm{e}_{i}\right\|$, ce qui montre alors que $\left(\mathrm{f}_{1}, \ldots, \mathrm{f}_{\nu}\right)$ est une famille $a$-orthogonale. Elle est donc libre et elle forme une base de $\mathrm{E}$ (ceci est un cas particulier du théorème 2.3.16 de [PGS]).

‡. Corps complet muni d'une valeur absolue discrète (non triviale). 
L'énoncé suivant est dû à Gaël Rémond.

Proposition 3.10. - Les fibrés adéliques hermitiens purs sur un corps de nombres $k$ sont exactement les fibrés vectoriels hermitiens sur $\operatorname{Spec} \mathcal{O}_{k}$.

Un fibré vectoriel hermitien sur $\operatorname{Spec} \mathcal{O}_{k}$ est toujours pur et l'intérêt de cette proposition réside dans le fait que la réciproque est vraie. La démonstration repose sur le lemme suivant.

LEMME 3.11. - Soit $\mathrm{K} / \mathrm{k}$ une extension galoisienne de corps valués ultramétriques. Notons $|\cdot|$ la valeur absolue sur $\mathrm{K}$ et supposons que $(\mathrm{K},|\cdot|)$ est sphériquement complet. Supposons également que l'extension des corps résiduels $\kappa(\mathrm{K}) / \kappa(\mathrm{k})$ est séparable. Soit $\mathrm{E}$ un $\mathrm{k}$-espace vectoriel de dimension finie et $\|\cdot\|$ une ultranorme sur $\mathrm{E} \otimes_{\mathrm{k}} \mathrm{K}$, invariante sous l'action du groupe de Galois $\mathrm{Gal}(\mathrm{K} / \mathrm{k})$. Alors toute base orthonormée de $(\mathrm{E},\|\cdot\|)$ reste une base orthonormée de $\left(\mathrm{E} \otimes_{\mathrm{k}} \mathrm{K},\|\cdot\|\right)$.

L'hypothèse d'invariance par Galois de la norme signifie que, pour toute k-base $\left(e_{1}, \ldots, e_{\nu}\right)$ de $\mathrm{E}$, pour tout $u \in \operatorname{Gal}(\mathrm{K} / \mathrm{k})$, pour tout $x_{1}, \ldots, x_{\nu} \in \mathrm{K}$, on a

$$
\left\|\sum_{i=1}^{\nu} \mathrm{e}_{i} \otimes u\left(x_{i}\right)\right\|=\left\|\sum_{i=1}^{\nu} \mathrm{e}_{i} \otimes x_{i}\right\| .
$$

L'hypothèse de séparabilité des corps résiduels est vérifiée lorsque $\kappa(\mathbf{k})$ est parfait, par exemple si k est une extension finie de $\mathbf{Q}_{p}$.

Démonstration. - On raisonne par récurrence sur la dimension $\nu \geq 1$. Le cas $\nu=1$ étant clair, supposons le lemme vrai pour tout espace de dimension $\leq \nu-1$. Soit $\mathrm{E}$ de dimension $\nu \geq 2$ et $\left(e_{1}, \ldots, e_{\nu}\right)$ une base orthonormée de $(E,\|\cdot\|)$. Il s'agit de montrer que

$$
\left\|\sum_{i=1}^{\nu} \mathrm{e}_{i} \otimes x_{i}\right\|=\max \left\{\left|x_{1}\right|, \ldots,\left|x_{\nu}\right|\right\}
$$

pour tout $x_{1}, \ldots, x_{\nu} \in \mathrm{K}$. Soit $\mathrm{F}$ le sous-espace de $\mathrm{E}$ engendré par $\mathrm{e}_{1}, \ldots, \mathrm{e}_{\nu-1}$. D'après l'hypothèse de récurrence appliquée à $(F,\|\cdot\|)$, la base orthonormée $\left(e_{1}, \ldots, e_{\nu-1}\right)$ de $F$ reste une base $K$ orthonormée de $\left(F \otimes_{k} K,\|\cdot\|\right)$. Comme $K$ est sphériquement complet, il existe $f \in E \otimes_{k} K \backslash\{0\}$ tel que $\operatorname{dist}\left(\mathrm{f}, \mathrm{F} \otimes_{\mathrm{k}} \mathrm{K}\right)=\|\mathrm{f}\|$. Quitte à diviser $\mathrm{f}$ par son coefficient devant $\mathrm{e}_{\nu}$ (nécessairement non nul), on peut supposer que $\mathrm{f}$ est de la forme $\mathrm{e}_{1} \otimes \lambda_{1}+\cdots+\mathrm{e}_{\nu-1} \otimes \lambda_{\nu-1}+\mathrm{e}_{\nu}$ avec $\lambda_{1}, \ldots, \lambda_{\nu-1} \in \mathrm{K}$. D'après le lemme 3.8, la base $\left(e_{1}, \ldots, e_{\nu-1}, f\right)$ de $E \otimes_{k} K$ est orthogonale. Ainsi on a

$$
1=\left\|\mathrm{e}_{\nu}\right\|=\max \left\{\|\mathrm{f}\|,\left|\lambda_{1}\right|, \ldots,\left|\lambda_{\nu-1}\right|\right\} .
$$

Supposons $\|\mathrm{f}\|<1$. Pour un élément $u$ du groupe de Galois de $\mathrm{K} / \mathrm{k}$, notons

$$
\mathrm{f}_{u}:=\mathrm{e}_{\nu}+\sum_{i=1}^{\nu-1} \mathrm{e}_{i} \otimes u\left(\lambda_{i}\right) .
$$

Comme la norme est invariante par Galois on a $\left\|\mathrm{f}_{u}\right\|=\|\mathrm{f}\|$ donc

$$
\max _{1 \leq i \leq \nu-1}\left|u\left(\lambda_{i}\right)-\lambda_{i}\right|=\left\|\mathrm{f}_{u}-\mathrm{f}\right\| \leq\|\mathrm{f}\|<1 .
$$

Comme l'extension $\kappa(\mathrm{K}) / \kappa(\mathrm{k})$ est séparable, elle est galoisienne et l'application canonique $\mathrm{Gal}(\mathrm{K} / \mathrm{k}) \rightarrow$ $\operatorname{Gal}(\kappa(\mathrm{K}) / \kappa(\mathrm{k}))$ qui à $u$ associe

$$
\bar{u}: x \bmod \mathrm{M} \mapsto u(x) \bmod \mathrm{M} \quad(\text { où } \mathrm{M}:=\{x \in \mathrm{K} ;|x|<1\})
$$

est surjective (voir par exemple [Ne II.9.9, p. 172]). Ainsi, pour tout $i \in\{1, \ldots, \nu-1\}$, l'inégalité $\left|u\left(\lambda_{i}\right)-\lambda_{i}\right|<1$ donne $\bar{u}\left(\lambda_{i} \bmod \mathrm{M}\right)=\lambda_{i} \bmod \mathrm{M}$. En faisant varier $u$, l'élément $\lambda_{i} \bmod \mathrm{M}$ est invariant par $\operatorname{Gal}(\kappa(\mathrm{K}) / \kappa(\mathrm{k}))$ et donc $\lambda_{i} \bmod \mathrm{M} \in \kappa(\mathrm{k})$, ce qui signifie qu'il existe $a_{i} \in \mathrm{k}$ tel que $\left|\lambda_{i}-a_{i}\right|<1$ (en particulier $\left|a_{i}\right| \leq 1$ ). En posant $\mathrm{g}:=\mathrm{e}_{\nu}+\sum_{i=1}^{\nu-1} \mathrm{e}_{i} \otimes a_{i}$, on a $\|\mathrm{f}-\mathrm{g}\|=$ $\max _{i}\left|\lambda_{i}-a_{i}\right|<1$ et $\|\mathrm{g}\|=1$ car $\left(\mathrm{e}_{1}, \ldots, \mathrm{e}_{\nu}\right)$ est une base orthonormée de $\mathrm{E}$. Ceci contredit l'inégalité ultramétrique $\|\mathrm{g}\| \leq \max (\|\mathrm{g}-\mathrm{f}\|,\|\mathrm{f}\|)$. Le cas $\|\mathrm{f}\|<1$ ne peut donc pas se produire. Ainsi on a $\|\mathrm{f}\|=1$ puis

$$
\operatorname{dist}\left(e_{\nu}, F \otimes_{k} K\right)=\operatorname{dist}\left(f, F \otimes_{k} K\right)=\|f\|=1=\left\|e_{\nu}\right\| .
$$

Par conséquent $\left(e_{1}, \ldots, e_{\nu}\right)$ est une base orthonormée de $\left(E \otimes_{k} K,\|\cdot\|\right)$ par la remarque qui suit le lemme 3.8 
Démonstration de la proposition 3.10. - Soit $\bar{E}=\left(E,\left(\|\cdot\|_{\bar{E}, \sigma}\right)_{\sigma}\right)$ un fibré adélique hermitien pur sur $k$. Fixons un plongement ultramétrique $\sigma: k \hookrightarrow \mathbf{C}_{p}$ et une base orthonormée $\left(\mathrm{e}_{1}, \ldots, \mathrm{e}_{\nu}\right)$ de $\left(E \otimes_{\sigma} k_{\sigma},\|\cdot\|_{\bar{E}, \sigma}\right)$. Soit $x_{1}, \ldots, x_{\nu} \in \mathbf{C}_{p}$ des éléments algébriques sur $\mathbf{Q}_{p}$ et $K / k$ une extension galoisienne finie qui contient ces nombres. Soit $\tau: K \hookrightarrow \mathbf{C}_{p}$ un plongement qui prolonge $\sigma$. On note $w$ la place de $K$ induite par $\tau$. L'extension $K_{\tau} / k_{\sigma}$ est galoisienne et, pour tout $u \in \operatorname{Gal}\left(K_{\tau} / k_{\sigma}\right)$ il existe un automorphisme continu $\iota: \mathbf{C}_{p} \rightarrow \mathbf{C}_{p}$ tel que $\tau \circ u=\iota \circ \tau$ (ces plongements correspondent tous à $w)$. Ainsi la norme $\|\cdot\|_{\bar{E}, \sigma}$ sur $\left(E \otimes_{\sigma} k_{\sigma}\right) \otimes_{k_{\sigma}} K_{\tau}$ est invariante par le groupe de Galois de $K_{\tau} / k_{\sigma}$ (au sens du lemme 3.11) car la collection de normes $\left(\|\cdot\|_{\overline{E_{K}}, \tau}\right)_{\tau \in w}$ est invariante par Galois (au sens de la définition [3.1). Le lemme 3.11 s'applique donc à (E, $\|\cdot\|)=\left(E \otimes_{\sigma} k_{\sigma},\|\cdot\|_{\bar{E}, \sigma}\right)$ et $\mathrm{K}=K_{\tau}$ (qui est une extension finie de $\mathbf{Q}_{p}$ donc sphériquement complet). On a ainsi

$$
\left\|\sum_{i=1}^{\nu} \mathrm{e}_{i} \otimes_{\sigma} x_{i}\right\|_{\bar{E}, \sigma}=\max _{1 \leq i \leq \nu}\left|x_{i}\right|_{p}
$$

Cette égalité est vraie pour tout $\left(x_{1}, \ldots, x_{\nu}\right) \in{\overline{\mathbf{Q}_{p}}}^{\nu}$, puis, par continuité, sur $\mathbf{C}_{p}^{\nu}$. La matrice de passage de $\left(\mathrm{e}_{1}, \ldots, \mathrm{e}_{\nu}\right)$ à une $k$-base $\left(e_{1}, \ldots, e_{\nu}\right)$ de $E$ fournit une matrice $A_{\sigma} \in \mathrm{GL}_{\nu}\left(k_{\sigma}\right)$ telle que

$$
\left\|\sum_{i=1}^{\nu} e_{i} \otimes_{\sigma} x_{i}\right\|=\left|A_{\sigma}\left(\begin{array}{c}
x_{1} \\
\vdots \\
x_{\nu}
\end{array}\right)\right|_{2, \sigma}
$$

De plus, l'invariance par Galois de $\left(\|\cdot\|_{\bar{E}, \sigma}\right)_{\sigma \in v}(v$ est la place de $k$ induite par $\sigma$ ) permet de choisir ces matrices de sorte que $A_{\iota \circ \sigma}=\iota\left(A_{\sigma}\right)$ pour tout automorphisme continu $\iota$ de $\mathbf{C}_{p}$. Le fibré $\bar{E}$ est donc un fibré vectoriel hermitien $\operatorname{sur} \operatorname{Spec} \mathcal{O}_{k}$.

Nous allons montrer maintenant que l'on peut approcher un fibré adélique hermitien par un fibré adélique hermitien pur, pourvu que l'on autorise une extension du corps de base.

Lemme 3.12. - Soit $\sigma: k \hookrightarrow \mathbf{C}_{p}$ un plongement ultramétrique d'un corps de nombres $k$ et $E$ un $k$-espace vectoriel de dimension $\nu \geq 1$. Soit $\|\cdot\|$ une ultranorme sur $E \otimes_{\sigma} \mathbf{C}_{p}$. Pour tout $\varepsilon>0$, $i l$ existe une extension finie $K$ de $k$, il existe une base $f_{1}, \ldots, f_{\nu}$ de $E \otimes_{k} K$ et un plongement $\tau: K \hookrightarrow \mathbf{C}_{p}$ qui prolonge $\sigma$ tels que, pour tous $x_{1}, \ldots, x_{\nu} \in \mathbf{C}_{p}$, on a

$$
\max _{1 \leq i \leq \nu}\left|x_{i}\right|_{p} \leq\left\|\sum_{i=1}^{\nu} f_{i} \otimes_{\tau} x_{i}\right\| \leq(1+\varepsilon) \max _{1 \leq i \leq \nu}\left|x_{i}\right|_{p} .
$$

Démonstration. - Par le lemme 3.8, il existe une base $\mathrm{e}_{1}, \ldots, \mathrm{e}_{\nu}$ de $E \otimes_{\sigma} \mathbf{C}_{p}$ telle que, pour tous $x_{1}, \ldots, x_{\nu} \in \mathbf{C}_{p}$, on a

$$
(1+\varepsilon)^{-1 / 2} \max _{1 \leq i \leq \nu}\left|x_{i}\right|_{p}\left\|\mathrm{e}_{i}\right\| \leq\left\|\sum_{i=1}^{\nu} x_{i} \mathrm{e}_{i}\right\| \leq \max _{1 \leq i \leq \nu}\left|x_{i}\right|_{p}\left\|\mathrm{e}_{i}\right\| .
$$

Comme les nombres algébriques sur $\mathbf{Q}$ sont denses dans $\mathbf{C}_{p}$, le lemme de perturbation permet de supposer qu'il existe une extension finie $K / k$ et un plongement $\tau: K \hookrightarrow \mathbf{C}_{p}$ qui prolonge $\sigma$ tels que $\mathrm{e}_{i} \in E \otimes_{\sigma} \tau(K)$. Considérons un entier $M \geq 1$ tel que $p^{1 / M} \leq(1+\varepsilon)^{1 / 2}$. Quitte à prendre une extension finie de $K$ on peut supposer que $p^{1 / M} \in K$. Par ailleurs, il existe $\alpha_{i} \in \mathbf{R}$ tel que $(1+\varepsilon)^{-1 / 2}\left\|\mathrm{e}_{i}\right\|=p^{\alpha_{i}}$. Soit $f_{i} \in E \otimes_{k} K$ tel que $f_{i} \otimes_{\tau} 1=p^{\left[\alpha_{i} M\right] / M} \mathrm{e}_{i}$. La famille $\left\{f_{1}, \ldots, f_{\nu}\right\}$ forme une base de $E \otimes_{k} K$ et la norme de $\sum_{i=1}^{\nu} f_{i} \otimes_{\tau} x_{i}$ vérifie l'encadrement voulu.

On notera que toute extension finie du corps $K$ donné par ce lemme convient encore.

COROllaire 3.13. - Soit $\bar{E}$ un fibré adélique hermitien sur $k$. Alors, pour tout nombre réel $\varepsilon>0$, il existe une extension finie $K$ de $k$ et un fibré adélique hermitien pur $\bar{E}_{\varepsilon}$ sur $K$, d'espace sousjacent $E \otimes_{k} K$, tels que, pour tout plongement $\tau: K \hookrightarrow \mathbf{C}_{p}$ qui prolonge $\sigma: k \hookrightarrow \mathbf{C}_{p}$, pour tout $x \in\left(E \otimes_{k} K\right) \otimes_{\tau} \mathbf{C}_{p}$, on $a$

$$
\|x\|_{\bar{E}_{\varepsilon}, \tau} \leq\|x\|_{E_{K}, \tau} \leq(1+\varepsilon)\|x\|_{\bar{E}_{\varepsilon}, \tau} .
$$

De plus, l'inégalité $\|\cdot\|_{E_{\varepsilon}, \tau} \leq\|\cdot\|_{E_{K}}, \tau$ 'est stricte que pour un nombre fini de plongements $\tau$. 
Démonstration. - Fixons une $k$-base $\left(e_{1}, \ldots, e_{\nu}\right)$ de $E$ et un sous-ensemble fini $S$ de $\mathcal{P}$ tel que, pour tout $p \notin S$, pour tout plongement $\sigma: k \hookrightarrow \mathbf{C}_{p}$, pour tout $\left(x_{1}, \ldots, x_{\nu}\right) \in \mathbf{C}_{p}^{\nu}$, on a

$$
\left\|e_{1} \otimes_{\sigma} x_{1}+\cdots+e_{\nu} \otimes_{\sigma} x_{\nu}\right\|_{\bar{E}, \sigma}=\max \left(\left|x_{1}\right|_{p}, \ldots,\left|x_{\nu}\right|_{p}\right)
$$

(première condition de la définition de fibré pré-adélique). On applique le lemme 3.12 à chacune des normes $\|\cdot\|_{E, \sigma}$ pour $\sigma: k \hookrightarrow \mathbf{C}_{p}$ avec $p \in S \backslash\{\infty\}$. L'on peut supposer que les extensions finies $K_{\sigma} / k$ que l'on obtient sont toutes les mêmes, égales à une extension galoisienne finie $K$ de $k$. Ainsi, pour chacun de ces $\sigma$, il existe un plongement $\tau_{\sigma}: K \hookrightarrow \mathbf{C}_{p}$ prolongeant $\sigma$ et une base $f_{1,1}, \ldots, f_{\nu, 1}$ de $E \otimes_{k} K$ (qui dépend de $\sigma$ ) tels que, pour tous $x_{1}, \ldots, x_{\nu} \in \mathbf{C}_{p}$, on a

$$
\max _{1 \leq i \leq \nu}\left|x_{i}\right|_{p} \leq\left\|\sum_{i=1}^{\nu} f_{i, 1} \otimes_{\tau_{\sigma}} x_{i}\right\|_{E_{K}, \tau_{\sigma}} \leq(1+\varepsilon) \max _{1 \leq i \leq \nu}\left|x_{i}\right|_{p} .
$$

Soit $a \in K$ tel que $K=k(a)$ et $\pi_{a}$ le polynôme minimal de $a$ sur $k$. Comme $K / k$ est galoisienne, l'on peut choisir des éléments $u_{1}=\mathrm{id}, u_{2}, \ldots, u_{g}$ du groupe de Galois de $K / k$ tels que les nombres $\tau_{\sigma} \circ u_{\ell}(a) \in \mathbf{C}_{p}$ pour $\ell \in\{1, \ldots, g\}$ soient chacune racine d'un des $g$ facteurs irréductibles de $\sigma\left(\pi_{a}\right)$ dans $k_{\sigma}[X]$. Les plongements $\tau: K \hookrightarrow \mathbf{C}_{p}$ au-dessus de $\sigma$ sont alors de la forme $\iota \circ \tau_{\sigma} \circ u_{\ell}$ avec $\iota$ automorphisme continu de $\mathbf{C}_{p}$ qui laisse fixe les éléments de $k_{\sigma}$ (on a rangé les plongements selon les places de $K$ au-dessus de $\sigma$ ). Considérons des éléments $\lambda_{i, j} \in K, 1 \leq i, j \leq \nu$, tels que $f_{i, 1}=\sum_{j=1}^{\nu} e_{j} \otimes_{k} \lambda_{i, j}$ pour tout $i \in\{1, \ldots, \nu\}$. Posons alors

$$
f_{i, \ell}:=\sum_{j=1}^{\nu} e_{j} \otimes_{k} u_{\ell}^{-1}\left(\lambda_{i, j}\right) \in E \otimes_{k} K
$$

Pour tous $x_{1}, \ldots, x_{\nu} \in \mathbf{C}_{p}$ et $\tau=\iota \circ \tau_{\sigma} \circ u_{\ell}$ et par définition des normes sur $\overline{E_{K}}$, on a alors

$$
\left\|\sum_{i=1}^{\nu} f_{i, \ell} \otimes_{\tau} x_{i}\right\|_{E_{K}, \tau}=\left\|\sum_{j=1}^{\nu} e_{j} \otimes_{\sigma}\left(\sum_{i=1}^{\nu} \iota \circ \tau_{\sigma}\left(\lambda_{i, j}\right) x_{i}\right)\right\|_{\bar{E}, \sigma} .
$$

Comme la norme $\|\cdot\|_{\bar{E}, \sigma}$ est invariante par Galois et comme $\iota \circ \sigma=\sigma$, on a

$$
\left\|\sum_{i=1}^{\nu} f_{i, \ell} \otimes_{\tau} x_{i}\right\|_{\overline{E_{K}}, \tau}=\left\|\sum_{i=1}^{\nu} f_{i, 1} \otimes_{\tau_{\sigma}} x_{i}\right\|_{\overline{E_{K}}, \tau_{\sigma}} .
$$

$\operatorname{Sur}\left(E \otimes_{k} K\right) \otimes_{\tau} \mathbf{C}_{p}$ (pour $\left.\tau=\iota \circ \tau_{\sigma} \circ u_{\ell}\right)$, définissons la norme $\|\cdot\|_{\bar{E}_{\varepsilon}, \tau}$ par la formule

$$
\left\|\sum_{i=1}^{\nu} f_{i, \ell} \otimes_{\tau} x_{i}\right\|_{E_{\varepsilon}, \tau}:=\max _{1 \leq i \leq \nu}\left|x_{i}\right|_{p}
$$

Le couple $\bar{E}_{\varepsilon}=\left(E \otimes_{k} K,\left(\|\cdot\|_{E_{\varepsilon}, \tau}\right)_{\tau}\right)$ forme un fibré hermitien pur sur $K$. De plus, l'encadrement $\|\cdot\|_{\bar{E}_{\varepsilon}, \tau} \leq\|\cdot\|_{\bar{E}_{K}, \tau} \leq(1+\varepsilon)\|\cdot\|_{\bar{E}_{\varepsilon}, \tau}$ résulte de (2) et (3).

Ce corollaire implique que, pour tout $\varepsilon>0$, pour tout fibré adélique hermitien $\bar{E}$ sur $k$, il existe une extension finie $K$ de $k$ et un fibré hermitien pur $\overline{E^{\prime}}$ sur $K$, avec $E^{\prime}=E \otimes_{k} K$, tels que, pour tout $x \in E \otimes_{k} \bar{k}$, on a

$$
h_{\overline{E^{\prime}}}(x) \leq h_{\bar{E}}(x) \leq h_{\overline{E^{\prime}}}(x)+\varepsilon
$$

De plus, on peut choisir $\overline{E^{\prime}}$ de sorte que

$$
h\left(\overline{E^{\prime}}\right) \leq h(\bar{E}) \leq h\left(\overline{E^{\prime}}\right)+\varepsilon \quad \text { et } \quad \widehat{\mu}_{\max }\left(\overline{E^{\prime}}\right)-\varepsilon \leq \widehat{\mu}_{\max }(\bar{E}) \leq \widehat{\mu}_{\max }\left(\overline{E^{\prime}}\right)
$$

car les inégalités de normes dans le corollaire 3.13 induisent des inégalités similaires pour les sousfibrés et les déterminants d'iceux. 
3.6. Normes d'opérateurs. - Soit $(\mathrm{K},|\cdot|)$ un corps valué complet. Soit $\left(\mathrm{E},\|\cdot\|_{\mathrm{E}}\right)\left(\right.$ resp. $\left.\left(\mathrm{F},\|\cdot\|_{\mathrm{F}}\right)\right)$ un $\mathrm{K}$-espace vectoriel normé de dimension $\nu \geq 1$ (resp. $\mu \geq 1$ ). Lorsque $\mathrm{K}$ est ultramétrique, on suppose que les normes sont des ultranormes. Soit a $: E \rightarrow F$ une application K-linéaire. L'équivalence des normes en dimension finie (qui découle par exemple de l'existence de bases 1/2-orthogonales) permet de poser la

DÉFINITION 3.14. - La norme d'opérateur de a est le nombre réel

$$
\|\mathrm{a}\|:=\sup \left\{\frac{\|\mathrm{a}(x)\|_{\mathrm{F}}}{\|x\|_{\mathrm{E}}} ; x \in \mathrm{E} \backslash\{0\}\right\} .
$$

Proposition 3.15. - Supposons que $\mathrm{K}$ est ultramétrique et que $\left(\mathrm{E},\|\cdot\|_{\mathrm{E}}\right)$ et $\left(\mathrm{F},\|\cdot\|_{\mathrm{F}}\right)$ possèdent des bases orthogonales, notées respectivement $\mathrm{e}=\left(\mathrm{e}_{1}, \ldots, \mathrm{e}_{\nu}\right)$ et $\mathrm{f}=\left(\mathrm{f}_{1}, \ldots, \mathrm{f}_{\mu}\right)$. Soit $\mathrm{A}=\left(\mathrm{a}_{i, j}\right) \in$ $\mathrm{M}_{\mu, \nu}(\mathrm{K})$ la matrice qui représente l'application linéaire a dans les bases et $\mathrm{f}$. Alors on a

$$
\|\mathrm{a}\|=\max \left\{\left|\mathrm{a}_{i, j}\right|\left\|\mathrm{f}_{i}\right\|_{\mathrm{F}} /\left\|\mathrm{e}_{j}\right\|_{\mathrm{E}} ; 1 \leq i \leq \mu, 1 \leq j \leq \nu\right\}
$$

Démonstration. - Notons $\alpha$ le maximum du membre de droite. Soit $x=\sum_{j=1}^{\nu} x_{j} \mathrm{e}_{j} \in \mathrm{E}$. On a

$$
\mathrm{a}(x)=\sum_{i=1}^{\mu}\left(\sum_{j=1}^{\nu} \mathrm{a}_{i, j} x_{j}\right) \mathrm{f}_{i}
$$

donc

$$
\|\mathrm{a}(x)\|_{\mathrm{F}} \leq \max _{i, j}\left|\mathrm{a}_{i, j} x_{j}\right|\left\|\mathrm{f}_{i}\right\|_{\mathrm{F}} \leq \alpha \max _{1 \leq j \leq \nu}\left|x_{j}\right|\left\|\mathrm{e}_{j}\right\|_{\mathrm{E}}=\alpha\|x\|_{\mathrm{E}}
$$

puis $\|\mathrm{a}\| \leq \alpha$. Réciproquement, considérons des indices $i_{0}, j_{0}$ tels que $\alpha=\left|\mathrm{a}_{i_{0}, j_{0}}\right|\left\|\mathrm{f}_{i_{0}}\right\|_{\mathrm{F}} /\left\|\mathrm{e}_{j_{0}}\right\|_{\mathrm{E}}$ et prenons $x=\mathrm{e}_{j_{0}}$. On a a $(x)=\sum_{i=1}^{\mu} \mathrm{a}_{i, j_{0}} \mathrm{f}_{i}$ et, par orthogonalité de $\mathrm{f}$, on en déduit

$$
\|\mathrm{a}(x)\|_{\mathrm{F}}=\max _{1 \leq i \leq \mu}\left|\mathrm{a}_{i, j_{0}}\right|\left\|\mathrm{f}_{i}\right\|_{\mathrm{F}} \geq\left|\mathrm{a}_{i_{0}, j_{0}}\right|\left\|\mathrm{f}_{i_{0}}\right\|_{\mathrm{F}}=\alpha\|x\|_{\mathrm{E}}
$$

puis $\|\mathrm{a}\| \geq \alpha$.

3.7. Puissances symétriques des fibrés adéliques hermitiens. - Étant donné des multiplets $m=\left(m_{1}, \ldots, m_{\nu}\right)$ et $n=\left(n_{1}, \ldots, n_{\nu}\right)$, on note $|m|$ la longueur $\sum_{i=1}^{\nu} m_{i}$ de $m$ et on désigne par $m$ ! (resp. $\left.m^{n}\right)$ le produit $m_{1} ! \cdots m_{\nu}$ ! (resp. $\left.m^{n}:=m_{1}^{n_{1}} \cdots m_{\nu}^{n_{\nu}}\right)$. Soit $\ell \geq 1$ un entier et $\bar{E}$ un fibré pré-adélique hermitien sur $k$. La puissance symétrique $S^{\ell}(E)$ est un quotient de $E^{\otimes \ell}$ (l'algèbre symétrique $\mathbf{S}(E)$ est le quotient de l'algèbre tensorielle $\mathbf{T}(E)$ par l'idéal engendré par les éléments $x \otimes y-y \otimes x)$. Cette structure quotient confère à $S^{\ell}(E)$ une structure de fibré pré-adélique hermitien $\overline{S^{\ell}(E)}$ induite par $\bar{E}^{\otimes \ell}$

Proposition 3.16. - Pour tout fibré adélique hermitien $\bar{E}$ sur $k$ de dimension $\nu \geq 1$, pour tout entier $\ell \geq 1$, on $a$

$$
\widehat{\mu}_{\max }\left(\overline{S^{\ell}(E)}\right) \leq \ell\left(\widehat{\mu}_{\max }(\bar{E})+2 \log \nu\right) .
$$

Démonstration. - Si $\bar{E}$ est un fibré vectoriel hermitien sur Spec $\mathcal{O}_{k}$, ce résultat est compris dans la proposition 8.4 de GR2. Le passage aux fibrés hermitiens quelconques s'effectue au moyen du corollaire 3.13, en utilisant l'invariance par extension des scalaires de la pente maximale de $\bar{E}$ (proposition 3.5).

Dans la suite, la puissance symétrique sera utilisée à la fois pour décrire l'ensemble des polynômes auxiliaires et pour décrire l'espace des dérivations. Si $E$ est un $k$-espace vectoriel, on rappelle que $E^{\vee}$ désigne l'espace dual $\operatorname{Hom}_{k}(E, k)$.

DÉfinition 3.17. - Un polynôme homogène $s$ sur $E$ de degré $\ell$ est un élément de $S^{\ell}\left(E^{\mathrm{v}}\right)$ et l'application polynomiale associée est l'application de $E$ dans $k$ qui envoie $x \in E$ sur $s(x)$. Plus généralement, si $n \in \mathbf{N}$ et si $E_{0}, \ldots, E_{n}$ sont des $k$-espaces vectoriels, un polynôme multihomogène $s$ de degré $\left(\ell_{0}, \ldots, \ell_{n}\right) \in \mathbf{N}^{n+1}$ est un élément de $\mathfrak{E}:=S^{\ell_{0}}\left(E_{0}^{\mathrm{v}}\right) \otimes \cdots \otimes S^{\ell_{n}}\left(E_{n}^{\mathrm{v}}\right)$ et l'application polynomiale associée est l'application de $\prod_{j=0}^{n} E_{j}$ dans $k$ qui envoie $\left(x_{0}, \ldots, x_{n}\right)$ sur $s\left(x_{0}, \ldots, x_{n}\right)$. 
Lorsque, pour tout $j \in\{0, \ldots, n\}$, l'espace $E_{j}$ possède une structure de fibré adélique hermitien $\overline{E_{j}}$ (il en est alors de même pour $E_{j}^{\mathrm{v}}$ avec les métriques duales), la norme de $s$ en une place $v$ de $k$ est calculée dans $\bigotimes_{j=0}^{n} \overline{S^{\ell_{j}}\left(E_{j}^{\mathrm{v}}\right)}$. Dans la pratique, si $\bar{E}$ est un fibré adélique hermitien pur sur $k$, si l'on fixe une base orthonormée $\mathrm{e}_{j}=\left(\mathrm{e}_{j, 1}, \ldots, \mathrm{e}_{j, \nu_{j}}\right)$ de $E_{j} \otimes_{\sigma} \mathbf{C}_{p}$ (ici $\nu_{j}=\operatorname{dim} E_{j}$ ), de base duale $\mathrm{e}_{j}^{\mathrm{v}}=\left(\mathrm{e}_{j, 1}^{\mathrm{v}}, \ldots, \mathrm{e}_{j, \nu_{j}}^{\mathrm{v}}\right)$, et si l'on écrit l'élément $s$ comme une somme

$$
s:=\sum_{i} p_{i} \prod_{j=0}^{n}\left(\mathrm{e}_{j}^{\mathrm{v}}\right)^{i_{j}}
$$

avec $i=\left(i_{0}, \ldots, i_{n}\right) \in \mathbf{N}^{\nu_{0}} \times \cdots \times \mathbf{N}^{\nu_{n}}, i_{j}$ de longueur $\ell_{j}$, et $p_{i} \in \mathbf{C}_{p}$, alors la norme $\|s\|_{\bar{E}, \sigma}$ de $s$ est

$$
\|s\|_{\overline{\mathfrak{E}}, \sigma}= \begin{cases}\left(\sum_{i}\left|\sigma\left(p_{i}\right)\right|^{2} \frac{i !}{\ell_{0} ! \cdots \ell_{n} !}\right)^{1 / 2} & \text { si } p=\infty \\ \max _{i}\left|p_{i}\right|_{p} & \text { si } p \neq \infty .\end{cases}
$$

Remarquons également que grâce à cette formule (6) et à l'inégalité de Cauchy-Schwarz, la somme $\sum_{i}\left|\sigma\left(p_{i}\right)\right|$ est plus petite que $\|s\|_{\overline{\mathfrak{E}}, \sigma} \times \prod_{j=0}^{n} \nu_{j}^{\ell_{j} / 2}$ (si $\sigma$ est complexe).

\section{Lemmes de Siegel approchés}

4.1. - La plupart des démonstrations de transcendance requiert l'utilisation d'une fonction auxiliaire qui doit satisfaire à un nombre fini de conditions linéaires. En général il s'agit de conditions d'annulations en des points particuliers avec des ordres de multiplicités dans certaines directions (on parle parfois de «points épaissis »). Aux prémices de la théorie (travaux d'Hermite et Lindemann par exemple), on exhibait hardiment une fonction auxiliaire explicite. Toutefois sont rapidement apparues les difficultés et les limitations inhérentes à cette approche presque impudique (\$). En filigrane dans les articles de Thue [T1,T2], l'on doit à Siegel d'avoir conceptualisé en 1929 l'idée qu'il suffisait de connaître une estimation de la «taille» de la fonction auxiliaire $F$. Demander l'annulation de $F$ en un nombre fini de points épaissis équivaut à réclamer que les coefficients de $F$ satisfassent à un système linéaire

$$
\forall i \in\{1, \ldots, \mu\}, \quad \sum_{j=1}^{\nu} a_{i, j} x_{j}=0
$$

d'inconnues $x_{1}, \ldots, x_{\nu}$. Lors de l'étude de la transcendance des valeurs des fonctions de Bessel [Si], Siegel formula l'énoncé précis suivant.

Lemme De Siegel. - Supposons que $\mu<\nu$ et que, pour tous $i, j$, on a $a_{i, j} \in \mathbf{Z}$. Soit $A=$ $\max _{i, j}\left|a_{i, j}\right|$. Alors il existe une solution $\left(x_{1}, \ldots, x_{\nu}\right) \in \mathbf{Z}^{\nu} \backslash\{0\}$ au système (77) telle que

$$
\max \left\{\left|x_{1}\right|, \ldots,\left|x_{\nu}\right|\right\} \leq 1+(\nu A)^{\frac{\mu}{\nu-\mu}} .
$$

Ce résultat découle simplement du principe des tiroirs de Dirichlet. Comme l'a remarqué Mignotte, il est possible de l'étendre à un système à coefficients algébriques (voir lemme 1.3.1 de [W1]). Cependant l'on s'est aperçu qu'un tel lemme n'était rien d'autre qu'une variante du premier théorème de Minkowski sur les corps convexes. Ce point de vue s'est révélé fécond en débouchant sur une version adélique du lemme de Siegel, démontrée par Bombieri \& Vaaler [BV] (voir [GR1, § 3.2] pour la constante $(1 / 2) \log \nu)$.

Lemme DE Bombieri \& VAALER. - Soit $k$ un corps de nombres de discriminant absolu $D_{k}$. Notons $\operatorname{rd}_{k}:=\left|D_{k}\right|^{1 /[k: \mathbf{Q}]}$ son discriminant racine. Soit $\bar{E}$ un fibré adélique hermitien pur sur $k$, de dimension $\nu \geq 1$. Alors il existe $x \in E \backslash\{0\}$ tel que

$$
h_{\bar{E}}(x) \leq-\widehat{\mu}(\bar{E})+\frac{1}{2}\left(\log \nu+\log \operatorname{rd}_{k}\right)
$$

§. Cependant, ce procédé reste encore très actuel au travers par exemple des approximants de Padé et des fonctions hypergéométriques car, comme l'avait noté Chudnovsky, il conduit souvent à de meilleurs résultats. 
Plusieurs développements de ce lemme existent. On peut demander que l'élément $x \in E \backslash\{0\}$ évite un nombre fini de sous-espaces vectoriels de $E$. Ceci constitue le fil directeur des articles GR1, G5. Mais la variante la plus importante est celle qui permet de s'affranchir de la dépendance en le discriminant du corps de nombres. On recherche une solution $x$ non nulle non pas dans $E$ mais dans $E \otimes \overline{\mathbf{Q}}$. Un tel lemme de Siegel est dit absolu. Roy \& Thunder ont obtenu un énoncé de ce type dans $\overline{\mathrm{RT}}$. Nous présentons ici un raffinement de leur résultat, signalé par David \& Philippon [DP], qui se déduit d'une inégalité de Zhang relative aux minima successifs d'une variété arithmétique [Zh.

Lemme de Siegel Absolu. - Soit $\bar{E}$ un fibré adélique hermitien sur $k$, de dimension $\nu \geq 1$. Alors il existe $x \in(E \otimes \overline{\mathbf{Q}}) \backslash\{0\}$ tel que

$$
h_{\bar{E}}(x) \leq-\widehat{\mu}(\bar{E})+\frac{1}{2} \log \nu
$$

L'énoncé de Roy \& Thunder donne $(\nu-1) / 4+\varepsilon$ au lieu de $\frac{1}{2} \log \nu$ lorsque $\bar{E}$ est pur. Avec la même hypothèse, Zhang montre que, pour tout $\varepsilon>0$, il existe $x \in E \otimes_{k} \overline{\mathbf{Q}}$ de hauteur plus petite que $-\widehat{\mu}(\bar{E})+\left(H_{\nu}-1\right) / 2+\varepsilon$, où $H_{\nu}=1+1 / 2+\cdots+1 / \nu$ est le nombre harmonique (la constante $(1 / 2) \log \nu$ étant un majorant strict de $\left(H_{\nu}-1\right) / 2$ lorsque $\left.\nu \geq 2\right)$. A priori valide pour les fibrés adéliques hermitiens purs sur $k$, cet énoncé reste vrai pour les fibrés adéliques hermitiens grâce au corollaire 3.13 (et aux conséquences qui suivent).

Si, autrefois, dans les démonstrations de mesures d'indépendance linéaire de logarithmes, le discriminant $\mathrm{du}$ corps de nombres était absorbé par des termes plus gros, aujourd'hui les mesures sont devenues assez fines pour que ce discriminant devienne un facteur limitant. C'est la présence de ce discriminant qui explique pourquoi l'énoncé principal de G1] s'exprime au moyen d'un maximum sur deux quantités. Comme il supprime cette imperfection, le lemme de Siegel absolu a pris une grande importance ces dernières années, ainsi que le prouve son utilisation dans plusieurs articles récents de la théorie des formes linéaires logarithmes $[\mathrm{DH}, \mathrm{AG}, \mathrm{G} 3$. Le fait que l'on ne maîtrise pas le (degré du) corps de nombres dans lequel vit la solution $x$ est sans conséquence car, en définitive, l'on est amené à effectuer une somme sur les différents plongements de ce corps, somme de laquelle émerge directement $h_{\bar{E}}(x)$. Néanmoins quelques soucis techniques peuvent surgir.

Tout d'abord, la hauteur de $\bar{E}$ peut s'avérer difficile à évaluer avec précision. Si, comme dans le lemme de Siegel original, l'espace vectoriel $E$ est défini par le système linéaire (7), la hauteur de $\bar{E}$ se calcule au moyen des mineurs maximaux de la matrice $A:=\left(a_{i, j}\right)_{i, j}$. En général, on estime la taille de ces mineurs avec l'inégalité d'Hadamard (le déterminant d'une famille de vecteurs est plus petit que le produit des normes hermitiennes de ces vecteurs), qui, in fine, fait ressortir la hauteur des $a_{i, j}$. Il arrive que les $a_{i, j}$ soient petits aux places archimédiennes (ce qui est très bien) mais avec un dénominateur trop grand aux places ultramétriques. L'astuce consiste alors à trouver un système

$$
\forall i \in\{1, \ldots, \mu\}, \quad \sum_{j=1}^{\nu} b_{i, j} x_{j}=0,
$$

équivalent à (7) et définissant ainsi le même espace vectoriel $E$, mais, où, cette fois-ci, les nombres algébriques $b_{i, j}$ sont petits aux places ultramétriques et de tailles quelconques aux autres places. Dans ce cas, la partie archimédienne de la hauteur de $\bar{E}$ est évaluée avec (7) et la partie ultramétrique avec (8). Cette technique fonctionne assez bien même si elle est parfois délicate à mettre en œuvre (voir p. ex. la démonstration de la proposition 4.15 de [G3]).

Une autre difficulté est que les coefficients $a_{i, j}$ qui se présentent naturellement peuvent ne pas être algébriques, même après renormalisation. Le système (7) ne possède alors en général aucune solution algébrique hormis $(0, \ldots, 0)$. C'est pourquoi il est plus raisonnable de demander au lemme de fournir une solution algébrique $\left(x_{1}, \ldots, x_{\nu}\right)$ non nulle au système d'inéquations

$$
\forall i \in\{1, \ldots, \mu\}, \quad\left|\sum_{j=1}^{\nu} a_{i, j} x_{j}\right| \leq \varepsilon
$$

(ici $\varepsilon$ est un nombre réel strictement positif). Un énoncé qui garantit l'existence d'une solution algébrique non nulle à ce système d'inéquations est appelé lemme de Siegel approché. En voici 
un exemple $(\mathbf{( \top )}$, extrait de l'article de Philippon \& Waldschmidt [PW1, qui a servi à la construction de la fonction auxiliaire de plusieurs articles marquants de la théorie des formes linéaires de logarithmes [PW1, PW2, H1, H2] (aussi utilisé dans [G1]) :

Lemme DE Siegel Approché. - Soit $\left(a_{i, j}\right), 1 \leq i \leq \mu, 1 \leq j \leq \nu$, une matrice de nombres complexes de rang $\rho$ et $A$ un nombre réel tel que $\max _{1 \leq i \leq \mu} \sum_{j=1}^{\nu}\left|a_{i, j}\right| \leq A$. Soit $H \in \mathbf{N} \backslash\{0\}$ et $\varepsilon \in] 0,+\infty[$ tels que

$$
\left(\frac{2 \mu H A}{\varepsilon}+1\right)^{2 \rho}<(H+1)^{\nu} .
$$

Alors il existe $\left(x_{1}, \ldots, x_{\nu}\right) \in \mathbf{Z}^{\nu} \backslash\{0\}$ tel que

$$
\max _{1 \leq j \leq \nu}\left|x_{j}\right| \leq H \quad \text { et } \max _{1 \leq i \leq \mu}\left|\sum_{j=1}^{\nu} a_{i, j} x_{j}\right| \leq \varepsilon .
$$

Tout comme pour le lemme de Siegel original, la démonstration repose sur le principe des tiroirs. S'il est facile de généraliser à un corps de nombres au moyen d'une Q-base $\left(\xi_{1}, \ldots, \xi_{D}\right)$ de ce corps, ceci fait intervenir la hauteur de cette base. Le corps considéré est souvent le corps de nombres dans lequel vivent tous les nombres algébriques de la démonstration. En particulier, pour les formes linéaires de logarithmes, la hauteur de $\left(\xi_{1}, \ldots, \xi_{D}\right)$ est liée aux paramètres $\log a$ et $\log b$. Pour faire disparaître la dépendance en le corps de nombres ambiant, l'on peut imaginer écrire un lemme de Siegel approché et absolu. Il s'avère qu'un lemme de Siegel (classique ou absolu) donne automatiquement un lemme de Siegel approché par déformation des normes $($ (II), , comme nous allons l'expliquer maintenant.

Soit $\bar{E}=\left(E,\left(\|\cdot\|_{\bar{E}, \sigma}\right)_{\sigma}\right)$ et $\bar{F}=\left(F,\left(\|\cdot\|_{\bar{F}, \sigma}\right)_{\sigma}\right)$ des fibrés adéliques hermitiens sur un corps de nombres $k$. Soit $S$ un ensemble fini (non vide) de plongements de $k$. Pour tout $\sigma \in S$, soit $\mathrm{a}_{\sigma}: E \otimes_{\sigma} \mathbf{C}_{p} \rightarrow F \otimes_{\sigma} \mathbf{C}_{p}$ une application $\mathbf{C}_{p}$-linéaire et posons a $:=\left(\mathrm{a}_{\sigma}\right)_{\sigma \in S}$. On considère sur $E \otimes_{\sigma} \mathbf{C}_{p}$ la norme tordue par $\mathrm{a}_{\sigma}$ :

$$
\forall x \in E \otimes_{\sigma} \mathbf{C}_{p}, \quad\|x\|_{\bar{E}_{\mathrm{a}}, \sigma}:=\operatorname{moy}_{\sigma}\left(\|x\|_{\bar{E}, \sigma},\left\|\mathrm{a}_{\sigma}(x)\right\|_{\bar{F}, \sigma}\right) .
$$

Si $\sigma \notin S$, on pose $\|\cdot\|_{E_{\mathrm{a}}, \sigma}:=\|\cdot\|_{\bar{E}, \sigma}$. Le couple $\bar{E}_{\mathrm{a}}=\left(E,\left(\|\cdot\|_{\bar{E}_{\mathrm{a}}, \sigma}\right)\right)$ forme un fibré pré-adélique hermitien sur $k$. Une condition suffisante pour qu'il soit adélique est que, pour tout $\sigma: k \hookrightarrow \mathbf{C}_{p}$ dans $S$, pour tout automorphisme continu $\iota: \mathbf{C}_{p} \rightarrow \mathbf{C}_{p}$, on a $\iota \circ \sigma \in S$ et les deux éléments de $F \otimes_{\iota \circ \sigma} \mathbf{C}_{p}$ que l'on obtient par les deux chemins du diagramme

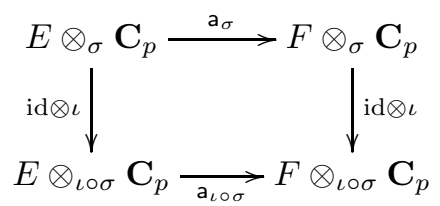

sont de même norme. De plus, si $x \in E$, on a $h_{\bar{E}}(x) \leq h_{\bar{E}_{\mathrm{a}}}(x)$ et $\left\|\mathrm{a}_{\sigma}(x)\right\|_{\bar{F}, \sigma} \leq\|x\|_{\bar{E}_{\mathrm{a}}, \sigma}$ pour $\sigma \in S$. Dès lors, savoir majorer finement $h_{E_{\mathrm{a}}}(x)$, pour un vecteur $x$ particulier, amène à établir un lemme de Siegel approché. On note $\left\|a_{\sigma}\right\|$ la norme d'opérateur de $a_{\sigma}$ (voir paragraphe 3.6) :

$$
\left\|\mathrm{a}_{\sigma}\right\|:=\sup \left\{\left\|\mathrm{a}_{\sigma}(x)\right\|_{\bar{F}, \sigma} /\|x\|_{\bar{E}, \sigma} ; x \in E \otimes_{\sigma} \mathbf{C}_{p} \backslash\{0\}\right\}
$$

Lemme de Siegel Approché AbSolu. - Soit $k$ un corps de nombres de degré $D$ et $S$ un ensemble fini de plongements de $k$. Soit $\bar{E}$ et $\bar{F}$ deux fibrés adéliques hermitiens sur $k$. Pour tout $\sigma \in S$, soit $\mathrm{a}_{\sigma}: E \otimes_{\sigma} \mathbf{C}_{p} \rightarrow F \otimes_{\sigma} \mathbf{C}_{p}$ une application $\mathbf{C}_{p}$-linéaire, de rang $\rho_{\sigma}$, et de norme d'opérateur $\left\|\mathrm{a}_{\sigma}\right\|$. On suppose que $\bar{E}_{\mathrm{a}}$ est un fibré adélique hermitien. Alors il existe $x \in(E \otimes \overline{\mathbf{Q}}) \backslash\{0\}$ tel que

$$
h_{\bar{E}_{\mathrm{a}}}(x) \leq \frac{1}{\nu D} \sum_{\sigma \in S} \rho_{\sigma} \log \operatorname{moy}_{\sigma}\left(1,\left\|\mathrm{a}_{\sigma}\right\|\right)+\frac{1}{2} \log \nu-\widehat{\mu}(\bar{E})
$$

ๆ. Cet exemple est dû en partie à Mignotte [Mi], qui s'est intéressé à la question dans les années 70 . On pourra aussi consulter le $\S 1.3$ du chapitre 1 de W1.

\|. A posteriori, nous nous sommes rendu compte que cette idée était déjà en substance dans l'article Mi] de Mignotte et qu'elle serait due à Mahler (voir Ma ). 
$\left(\right.$ ici $\operatorname{moy}_{\sigma}=\operatorname{moy}_{p}$ ò̀ $p \in \mathcal{P}$ est celui correspondant $\grave{a} \sigma: k \hookrightarrow \mathbf{C}_{p}$ ).

Une caractéristique importante du majorant est la présence des quotients $\rho_{\sigma} / \nu$, qui seront petits dans le contexte des formes linéaires de logarithmes (voir proposition 5.14). Dans un souci d'efficacité, nous n'avons pas séparé les contributions des normes de $x$ en les différents plongements de $\overline{\mathbf{Q}}$, en exprimant simplement le résultat en termes de hauteur (globale) de $x$. Par ailleurs une manière de procéder pour faire en sorte que $\bar{E}_{\mathrm{a}}$ soit un fibré adélique hermitien est de considérer un ensemble $S_{0}$ de plongements $\sigma: k \hookrightarrow \mathbf{C}_{p}$ qui n'induisent pas la même valuation. Pour un plongement conjugué à $\sigma$, c'est-à-dire pour $\sigma^{\prime}=\iota \circ \sigma$ avec $\iota$ automorphisme continu de $\mathbf{C}_{p}$, on choisit a $a_{\sigma^{\prime}}$ de sorte que le diagramme (10) vérifie la condition d'isométrie et l'on prend $S=\left\{\sigma^{\prime} ; \sigma \in S_{0}\right\}$ (le cardinal de $S$ est $\left.\sum_{\sigma \in S_{0}}\left[k_{\sigma}: \mathbf{Q}_{p}\right]\right)$.

4.2. Démonstration du lemme de Siegel approché absolu. — En vertu du lemme de Siegel absolu de Zhang appliqué à $\bar{E}_{\mathrm{a}}$, il suffit de montrer que

$$
-\widehat{\mu}\left(\bar{E}_{\mathrm{a}}\right) \leq \frac{1}{\nu D} \sum_{\sigma \in S} \rho_{\sigma} \log \operatorname{moy}_{\sigma}\left(1,\left\|\mathrm{a}_{\sigma}\right\|\right)-\widehat{\mu}(\bar{E}) .
$$

Par définition du degré adélique, pour toute $k$-base $e_{1}, \ldots, e_{\nu}$ de $E$, on a

$$
\widehat{\mu}\left(\bar{E}_{\mathrm{a}}\right)-\widehat{\mu}(\bar{E})=-\frac{1}{\nu D} \sum_{p \in \mathcal{P}} \sum_{\sigma: k \hookrightarrow \mathbf{C}_{p}} \log \frac{\left\|e_{1} \wedge \cdots \wedge e_{\nu}\right\|_{\overline{\operatorname{det} E_{\mathrm{a}}}, \sigma}}{\left\|e_{1} \wedge \cdots \wedge e_{\nu}\right\|_{\overline{\operatorname{det} E}, \sigma}} .
$$

Dans cette somme, seuls les $\sigma \in S$ interviennent, les autres donnant des termes nuls. De plus, à $\sigma$ fixé, chacun des quotients $\left\|e_{1} \wedge \cdots \wedge e_{\nu}\right\|_{\overline{\operatorname{det} E_{\mathrm{a}}}, \sigma} /\left\|e_{1} \wedge \cdots \wedge e_{\nu}\right\|_{\overline{\operatorname{det} E}, \sigma}$ est indépendant du choix de la base $e_{1}, \ldots, e_{\nu}$ de $E \otimes_{\sigma} \mathbf{C}_{p}$. Pour calculer ce quotient, supposons dans un premier temps que $\sigma$ est ultramétrique et considérons un nombre réel $t \in] 0,1]$ et une base $t$-orthogonale $\mathrm{e}_{1}, \ldots, \mathrm{e}_{\nu}$ de $E \otimes_{\sigma} \mathbf{C}_{p}$ telle que $\mathrm{e}_{\rho_{\sigma}+1}, \ldots, \mathrm{e}_{\nu}$ soit une base de ker $\mathrm{a}_{\sigma}$ [PGS, corollaire 2.3.21]. Alors, d'une part, comme la norme sur le déterminant est une norme quotient, on a

$$
\left\|\mathrm{e}_{1} \wedge \cdots \wedge \mathrm{e}_{\nu}\right\| \frac{}{\operatorname{det} E_{\mathrm{a}}, \sigma} \leq \prod_{i=1}^{\nu}\left\|\mathrm{e}_{i}\right\|_{\bar{E}_{\mathrm{a}}, \sigma} \leq\left(\prod_{i=1}^{\nu}\left\|\mathrm{e}_{i}\right\|_{\bar{E}, \sigma}\right) \operatorname{moy}_{\sigma}\left(1,\left\|\mathrm{a}_{\sigma}\right\|\right)^{\rho_{\sigma}} .
$$

D'autre part, la base $\left\{\mathrm{e}_{i_{1}} \otimes \cdots \otimes \mathrm{e}_{i_{\nu}}, 1 \leq i_{1}, \ldots, i_{\nu} \leq \nu\right\}$ de $\left(E \otimes_{\sigma} \mathbf{C}_{p}\right)^{\otimes \nu}$ est $t^{\nu}$-orthogonale [PGS, corollaire 10.2.10, (vi)]. Si un vecteur $\mathrm{x} \in\left(E \otimes_{\sigma} \mathbf{C}_{p}\right)^{\otimes \nu}$ a pour coordonnées $\left(\mathrm{x}_{i_{1}, \ldots, i_{\nu}}\right)$ dans cette base alors son image dans det $E \otimes_{\sigma} \mathbf{C}_{p}$ est le vecteur $\left(\sum_{s \in \mathfrak{S}_{\nu}} \varepsilon(s) \mathrm{x}_{s(1), \ldots, s(\nu)}\right) \mathrm{e}_{1} \wedge \cdots \wedge \mathrm{e}_{\nu}$. On en déduit que, pour tout $\mathrm{x} \in\left(E \otimes_{\sigma} \mathbf{C}_{p}\right)^{\otimes \nu}$, on a

$$
\left\|\mathrm{e}_{1} \otimes \cdots \otimes \mathrm{e}_{\nu}+\mathrm{x}\right\| \frac{}{\operatorname{det} E, \sigma} \geq t^{\nu}\left(\prod_{i=1}^{\nu}\left\|\mathrm{e}_{i}\right\|_{\bar{E}, \sigma}\right) \max _{s \in \mathfrak{S}_{\nu} \backslash\{\text { id }\}}\left\{\left|1+\mathrm{x}_{1, \ldots, \nu}\right|_{p},\left|\mathrm{x}_{s(1), \ldots, s(\nu)}\right|_{p}\right\} .
$$

Si, de plus, l'image de $\mathrm{x}$ dans $\operatorname{det} E \otimes_{\sigma} \mathbf{C}_{p}$ est nulle alors $\sum_{s \in \mathfrak{S}_{\nu}} \varepsilon(s) \mathrm{x}_{s(1), \ldots, s(\nu)}=0$. De cette relation et par inégalité ultramétrique, l'on déduit que le maximum ci-dessus est supérieur à 1 puis que

$$
\left\|\mathrm{e}_{1} \wedge \cdots \wedge \mathrm{e}_{\nu}\right\|_{\overline{\operatorname{det} E}, \sigma} \geq t^{\nu} \prod_{i=1}^{\nu}\left\|\mathrm{e}_{i}\right\|_{\bar{E}, \sigma} .
$$

On a donc

$$
\frac{\left\|\mathrm{e}_{1} \wedge \cdots \wedge \mathrm{e}_{\nu}\right\|_{\overline{\operatorname{det} E_{\mathrm{a}}}, \sigma}}{\left\|\mathrm{e}_{1} \wedge \cdots \wedge \mathrm{e}_{\nu}\right\|_{\overline{\operatorname{det} E}, \sigma}} \leq t^{-\nu} \operatorname{moy}_{\sigma}\left(1,\left\|\mathrm{a}_{\sigma}\right\|\right)^{\rho_{\sigma}} .
$$

Lorsque $\sigma$ est archimédien, la même inégalité reste valide en choisissant la base $\left(\mathrm{e}_{1}, \ldots, \mathrm{e}_{\nu}\right)$ orthonormée (la preuve se simplifie car $t=1$ convient). Ceci conduit à l'estimation

$$
-\widehat{\mu}\left(\bar{E}_{\mathrm{a}}\right)+\widehat{\mu}(\bar{E}) \leq \frac{1}{\nu D} \sum_{\sigma \in S} \rho_{\sigma} \log \operatorname{moy}_{\sigma}\left(1,\left\|\mathrm{a}_{\sigma}\right\|\right)-\frac{\operatorname{card} S}{D} \log t .
$$

On conclut en faisant tendre $t$ vers 1 . 


\section{Démonstration du théorème 2.1}

5.1. Canevas de la démonstration. - Nous utilisons la méthode de Baker non pas avec des fonctions auxiliaires, ni avec des déterminants d'interpolation, ni avec la méthode des pentes. En réalité, nous faisons un mélange entre la première et la dernière de ces méthodes, c'est-à-dire que nous passons par la construction d'une section auxiliaire d'un certain fibré adélique hermitien. À chaque étape de la démonstration nous conservons l'aspect intrinsèque des données. De la sorte nous bénéficions de la souplesse de la méthode des fonctions auxiliaires et de la possibilité d'accéder naturellement aux constantes numériques de la méthode des pentes. Concrètement, après avoir modifié les données initiales de manière à être en mesure d'utiliser la réduction d'Hirata-Kohno et le procédé de changement de variables de Chudnovsky, nous construisons un fibré adélique hermitien $\bar{E}$. L'espace vectoriel $E$ sous-jacent est un espace de polynômes en plusieurs variables, auquel sont adjointes des normes en tous les plongements de $k$. Ce fibré adélique $\bar{E}$ a la particularité d'avoir une $\sigma_{0}$-norme «tordue », ce qui constitue une des nouveautés de ce texte. Nous construisons alors un élément $s \neq 0$ de $E$ de petite hauteur au moyen du lemme de Siegel absolu. Le choix des paramètres et le lemme de multiplicités de Philippon assurent qu'il existe un jet de $s$ le long de $W$ (sous-espace construit à partir de $\left.W_{0}\right)$ en un multiple de $\mathrm{p}=\left(u_{0}, \alpha_{1}, \ldots, \alpha_{n}\right)$ qui est non nul. Ensuite nous évaluons la hauteur de ce jet, en distinguant les normes relatives aux plongements ultramétriques de celles relatives aux plongements archimédiens. Le comportement du jet en $\sigma_{0}$ et tous ses conjugués est étudié à part. La majoration de sa norme repose sur une extrapolation sur les dérivations (cas périodique) ou sur les multiples de $\mathrm{p}$ (cas non périodique), qui a été préparée par la construction de $s$. C'est à cet endroit qu'apparaissent les valeurs absolues des formes linéaires que nous cherchons à évaluer. Pour conclure, nous utilisons une variante de l'inégalité de Liouville (lemme 3.6) pour minorer la hauteur de ce jet, qui fait intervenir la pente maximale arakelovienne de l'espace naturel dans lequel vit le jet considéré.

5.2. Réductions. - Pour démontrer le théorème 2.1 l'on peut supposer que

(i) $\left\{u_{1}, \ldots, u_{n}\right\}$ est une famille libre sur $\mathbf{Q}$,

(ii) $\left\{\ell_{1}, \ldots, \ell_{t}\right\}$ est une famille libre du dual $\left(k^{n}\right)^{v}$,

(iii) $\left|\left(\beta_{1,0}, \ldots, \beta_{t, 0}\right)\right|_{2, \sigma_{0}} \leq 1$ lorsque $\sigma_{0}$ est archimédien $(* *)$,

(iv) si $n=1$ (et donc $t=1$ ) alors $\beta_{1,0} \neq 0$.

En effet, considérons un ensemble $I$ tel que $\left(u_{i}\right)_{i \in I}$ soit une base du Q-espace vectoriel engendré par $u_{1}, \ldots, u_{n}$. Posons $J=\{1, \ldots, n\} \backslash I$. Pour $j \in J$, la famille $\left\{u_{i}\right\}_{i \in I} \cup\left\{u_{j}\right\}$ est liée sur Q. Le lemme 7.19 de [W3, p. 222] assure l'existence de nombres rationnels $\theta_{j, i}$ tels que $u_{j}=\sum_{i \in I} \theta_{j, i} u_{i}$ et

$$
h\left(\theta_{j, i}\right) \leq(n-1) \log \left(11(n-1) D^{3}\right)+\log \prod_{l=1}^{n} \log a_{l}
$$

pour tous $j, i$. En utilisant la définition de $\mathfrak{a}$, on a

$$
h\left(\theta_{j, i}\right) \leq(n-1) \log (11(n-1))+3(n-1)\left(\log \frac{D}{\log \mathfrak{e}}+\log \log \mathfrak{e}\right)+n \frac{\mathfrak{a} \log \mathfrak{e}}{D}
$$

puis, comme $\frac{\mathfrak{a} \log \mathfrak{e}}{D} \geq \max \left(1, \log \frac{D}{\log \mathfrak{e}}\right)$, on trouve

$$
\begin{aligned}
h\left(\theta_{j, i}\right) & \leq((n-1) \log (11(n-1))+4 n-3)\left(\frac{\mathfrak{a} \log \mathfrak{e}}{D}+\log \log \mathfrak{e}\right) \\
& \leq 2 n^{2}\left(\frac{\mathfrak{a} \log \mathfrak{e}}{D}+\log \log \mathfrak{e}\right) .
\end{aligned}
$$

Par ailleurs, chaque $\ell_{\mathrm{j}}(u), 1 \leq \mathrm{j} \leq t$, est la valeur d'une forme linéaire $L_{\mathrm{j}}$ en les $u_{i}, i \in I$, avec des coefficients de la forme

$$
\beta_{\mathrm{j}, i}^{\prime}:=\beta_{\mathrm{j}, i}+\sum_{m \notin I} \beta_{\mathrm{j}, m} \theta_{m, i} .
$$

**. Cette hypothèse sera utile uniquement pour le cas périodique, $\S 5.12 .2$ 
Pour un tel coefficient on a

$$
\left|\beta_{\mathrm{j}, i}^{\prime}\right|_{\sigma} \leq n^{\epsilon_{\sigma}} \max \left(1,\left|\beta_{\mathrm{j}, i}\right|_{\sigma}\right) \prod_{m \notin I} \max \left(1,\left|\beta_{\mathrm{j}, m}\right|_{\sigma}\right) \max \left(1,\left|\theta_{m, i}\right|_{\sigma}\right)
$$

ce qui conduit à la borne de la hauteur

$$
\begin{aligned}
h\left(\beta_{\mathbf{j}, i}^{\prime}\right) & \leq \log n+n \max _{m, \ell}\left\{h\left(\beta_{m, \ell}\right)\right\}+n \max _{m, i}\left\{h\left(\theta_{m, i}\right)\right\} \\
& \leq \frac{(n+\log n) \log b}{D}+2 n^{3}\left(\frac{\mathfrak{a} \log \mathfrak{e}}{D}+\log \log \mathfrak{e}\right) \\
& \leq \frac{2 n^{3}}{D}(\log b+\mathfrak{a} \log \mathfrak{e}+D \log \log \mathfrak{e}) .
\end{aligned}
$$

De cette famille $\left\{L_{1}, \ldots, L_{t}\right\}$ de formes linéaires sur $k^{I}$, l'on peut extraire une famille libre maximale, qui comporte exactement $s=\operatorname{dim} \mathrm{T}_{u}-\operatorname{dim}\left(W_{0} \cap \mathrm{T}_{u}\right)$ éléments. Notons $\mathrm{S}$ le sous-ensemble de $\{1, \ldots, t\}$ qui indexe la famille libre choisie. Pour tout $j \in\{1, \ldots, t\}$, on a $\Lambda_{j}=\beta_{j, 0}+L_{j}\left(\left(u_{i}\right)_{i \in I}\right)$ et le maximum des $\left|\Lambda_{j}\right|_{p_{0}}$ que l'on cherche à minorer est plus grand que $\max _{\mathbf{s} \in \mathrm{S}}\left\{\left|\beta_{\mathbf{s}, 0}+L_{\mathbf{s}}\left(\left(u_{i}\right)_{i \in I}\right)\right|_{p_{0}}\right\}$. De la sorte l'on s'est donc bien ramené aux conditions (i) et (ii) ci-dessus. Pour la condition (iii), il suffit de diviser tous les nombres $\beta_{i, j}$ par le premier entier supérieur à $\left|\left(\beta_{1,0}, \ldots, \beta_{t, 0}\right)\right|_{2, \sigma_{0}}$. Cet entier est plus petit que $n b$. Enfin pour la condition (iv), lorsque $n=1$ et $\beta_{1,0}=0$, il n'y a qu'une seule forme linéaire $\Lambda_{1}=\beta_{1,1} u_{1}$. L'inégalité de Liouville donne $\log \left|\beta_{1,1}\right|_{\sigma_{0}} \geq-D h\left(\beta_{1,1}\right)$. De plus, si $\sigma_{0}$ est ultramétrique, on a $\left|u_{1}\right|_{\sigma_{0}}=\left|\alpha_{1}-1\right|_{\sigma_{0}}$ et, si $\sigma_{0}$ est archimédien, on a

$$
\left|\alpha_{1}-1\right|_{\sigma_{0}}=\left|u_{1} \int_{0}^{1} e^{t u_{1}} \mathrm{~d} t\right|_{\sigma_{0}} \leq\left|u_{1}\right|_{\sigma_{0}} \int_{0}^{1} e^{t} \mathrm{~d} t \leq 2\left|u_{1}\right|_{\sigma_{0}}
$$

lorsque $\left|u_{1}\right|_{\sigma_{0}} \leq 1$. De plus, dans tous les cas, $\log \left|\alpha_{1}-1\right|_{\sigma_{0}} \geq-D h\left(\alpha_{1}-1\right) \geq-D\left(h\left(\alpha_{1}\right)+\log 2\right)$. On obtient donc

$$
\begin{aligned}
\log \left|\Lambda_{1}\right|_{\sigma_{0}} & \geq-\log b-D\left(\log a_{1}+2 \log 2\right) \\
& \leq-2(\log b+\log \mathfrak{e})\left(1+\frac{D \log a_{1}}{\log \mathfrak{e}}\right)
\end{aligned}
$$

et le théorème 2.1 est vrai. On peut donc supposer la condition (iv) vérifiée.

Les estimations faites des hauteurs des coefficients des $L_{\mathrm{s}}$ montrent que le théorème 2.1 découle de l'énoncé suivant (les notations sont celles du théorème 2.1).

ThÉORÈme 5.1. - Supposons que $\left\{u_{1}, \ldots, u_{n}\right\}$ et $\left\{\ell_{1}, \ldots, \ell_{t}\right\}$ sont des familles libres sur $\mathbf{Q}$. Alors, pour tout $j \in\{1, \ldots, t\}$, on $a \Lambda_{j} \neq 0$ et

$$
\log \max _{1 \leq j \leq t}\left|\Lambda_{j}\right|_{p_{0}} \geq-(4 n)^{90 n^{2}} \mathfrak{a}^{1 / t}(\log b+\mathfrak{a} \log \mathfrak{e}) \prod_{j=1}^{n}\left(1+\frac{D \log a_{j}}{\log \mathfrak{e}}\right)^{1 / t} .
$$

De plus, si $t=1$ et $\beta_{1,0} \neq 0$, la quantité $\log b+\mathfrak{a} \log \mathfrak{e}$ qui est dans le minorant peut être remplacée par $\log b+\log \mathfrak{e}+D \log \mathfrak{a}$.

Le fait qu'aucun des $\Lambda_{j}$ n'est nul est une conséquence du théorème de Baker qui affirme que la famille $\left\{1, u_{1}, \ldots, u_{n}\right\}$ est libre sur $\overline{\mathbf{Q}}$ lorsque $\left\{u_{1}, \ldots, u_{n}\right\}$ l'est sur $\mathbf{Q}$. La constante $(4 n)^{91 n^{2}}$ qui est dans le théorème 2.1 est un majorant simple de $(4 n)^{90 n^{2}}\left(2 n^{3}+1\right)$, le terme $2 n^{3}$ venant de la majoration des hauteurs des $\beta_{\mathrm{j}, i}^{\prime}$ donnée plus haut. Le théorème 1.1 donné dans l'introduction est une conséquence immédiate de l'énoncé 5.1 en choisissant $a_{j}=a$ pour tout $j \in\{1, \ldots, n\}$. Il faut toutefois prendre garde que la valeur de a dans le théorème 1.1 n'est pas tout à fait celle que l'on obtiendrait en particularisant la valeur de $\mathfrak{a}$ qui est dans le théorème 2.1 car $\log \prod_{j=1}^{n} a_{j}=n \log a$. C'est la raison pour laquelle nous avons la constante $(4 n)^{91 n^{2}}$ dans le théorème 1.1. constante qui majore $(4 n)^{90 n^{2}} \max \{1, \log n\}^{2}$.

La suite de l'article concerne la démonstration du théorème 5.1. En particulier, dans toute la suite, nous supposerons que les familles $\left\{u_{1}, \ldots, u_{n}\right\}$ et $\left\{\ell_{1}, \ldots, \ell_{t}\right\}$ sont des familles libres sur $\mathbf{Q}$. 
5.3. Préparatifs. - Avant de commencer la démonstration du théorème 5.1, il nous est utile de modifier les données brutes du paragraphe 2.1.

Rappelons que $W_{0}$ désigne le sous-espace vectoriel de $k^{n}$ intersection des noyaux des formes linéaires $\ell_{i}, i \in\{1, \ldots, t\}$. Soit $G_{0}$ le spectre de l'algèbre symétrique $\mathbf{S}\left(\left(k^{n} / W_{0}\right)^{\mathrm{v}}\right)$. C'est un schéma en groupes affine sur Spec $k$, dont l'espace tangent à l'origine s'identifie canoniquement au quotient $k^{n} / W_{0}$. Notons $G$ le $k$-groupe algébrique linéaire $G_{0} \times \mathbf{G}_{\mathrm{m}}^{n}$. Soit $\lambda: k^{n} \rightarrow k^{n} / W_{0}$ la projection canonique et $W$ le sous-espace de l'espace tangent à l'origine $t_{G}=\left(k^{n} / W_{0}\right) \oplus k^{n}$ défini comme l'ensemble des vecteurs de la forme $(\lambda(y), y)$ avec $y \in k^{n}$. Le fibré adélique hermitien $\left(k^{n},|\cdot|_{2}\right)$ confère à $k^{n} / W_{0}$ et à $t_{G}$ des structures adéliques hermitiennes, respectivement par quotient et par somme directe orthogonale. Nous noterons $\overline{k^{n} / W_{0}}$ et $\overline{t_{G}}$ les fibrés adéliques hermitiens ainsi obtenus. Comme sous-espace vectoriel de $\left(k^{n},\left.|\cdot|\right|_{2}\right)$, l'espace $W_{0}$ hérite d'une structure de fibré adélique hermitien $\overline{W_{0}}$ et l'on note $h\left(\overline{W_{0}}\right)$ sa hauteur d'Arakelov au sens du $\S 3$ Par hermitianité, cette quantité est aussi le degré d'Arakelov de $\overline{k^{n} / W_{0}}$. À une constante qui ne dépend que de $n$ près, elle est bornée par $\max _{i, j}\left\{1, h\left(\beta_{i, j}\right)\right\}$, terme qui apparaît dans la définition de $\log b$ du théorème 2.1. Plus précisément on a la

Proposition 5.2. — La hauteur d'Arakelov de $\overline{W_{0}}$ vérifie l'inégalité

$$
D \max \left\{1, h\left(\overline{W_{0}}\right)\right\} \leq n^{3} \log b .
$$

Démonstration. - Si $n=t$ alors $W_{0}=\{0\}$ et la proposition est vraie. Nous supposons maintenant $t \leq n-1$. L'application $k^{n} \rightarrow k^{t}$ qui à $z=\left(z_{1}, \ldots, z_{n}\right)$ associe $\left(\ell_{1}(z), \ldots, \ell_{t}(z)\right)$ se factorise en un isomorphisme $\varphi: k^{n} / W_{0} \rightarrow k^{t}$ de $k$-espaces vectoriels. Pour tout $i \in\{1, \ldots, t\}$ et pour tout plongement $\sigma: k \hookrightarrow \mathbf{C}_{p}$, l'inégalité de Cauchy-Schwarz fournit la majoration

$$
\left|\ell_{i}\left(z_{1}, \ldots, z_{n}\right)\right|_{\sigma} \leq\left\|\ell_{i}\right\|_{\left(k^{n},|\cdot|_{2}\right)^{v}, \sigma}\|\bar{z}\|_{\overline{k^{n} / W_{0}}, \sigma}
$$

avec

$$
\left\|\ell_{i}\right\|_{\left(k^{n},|\cdot|_{2}\right)^{\mathrm{v}}, \sigma}=\operatorname{moy}_{p}\left(\left|\beta_{i, 1}\right|_{\sigma}, \ldots,\left|\beta_{i, n}\right|_{\sigma}\right) .
$$

Par conséquent, la norme d'opérateur $\|\varphi\|_{\sigma}$ de $\varphi$ entre $\overline{k^{n} / W_{0}}$ et $\left(k^{t},|\cdot|_{2}\right)$ est plus petite que $\left|\left(\beta_{i, j}\right)_{i, j}\right|_{2, \sigma}$. Ainsi on a

$$
\begin{aligned}
h\left(\overline{W_{0}}\right)=\widehat{\operatorname{deg}}_{\mathrm{n}}\left(\overline{k^{n} / W_{0}}\right) & \leq \widehat{\operatorname{deg}}_{\mathrm{n}}\left(k^{t},|\cdot|_{2}\right)+\frac{t}{D} \sum_{p \in \mathcal{P}} \sum_{\sigma: k \hookrightarrow \mathbf{C}_{p}} \log \|\varphi\|_{\sigma} \\
& \leq 0+t h_{\left(k^{n t},|\cdot|_{2}\right)}\left(\left(\beta_{i, j}\right)_{i, j}\right) \\
& \leq \frac{t}{2} \log (n t)+n t^{2} \frac{\log b}{D}
\end{aligned}
$$

(la première inégalité est une inégalité de pentes classique, voir p. ex. le lemme 6.3 de [G4]). On conclut au moyen des majorations suivantes:

$$
\begin{aligned}
\frac{t}{2} \log (n t)+n t^{2} & \leq \frac{(n-1)}{2} \log (n(n-1))+n(n-1)^{2} \\
& \leq n \log n+n^{3}-n(2 n-1) \\
& \leq n^{3}-n^{2}+n \leq n^{3}
\end{aligned}
$$

car $\log n \leq n$.

L'isomorphisme $\varphi$ défini ci-dessus permet de définir $u_{0}^{\prime}:=\varphi^{-1}\left(u_{0}\right) \in k^{n} / W_{0}$. Dans la suite, nous confondrons $u_{0}^{\prime}$ avec $u_{0}$. Nous ne garderons que la notation $u_{0}$, le contexte permettant de distinguer s'il s'agit de $u_{0}^{\prime} \in k^{n} / W_{0}$ ou bien de $u_{0} \in k^{t}$.

Par ailleurs, plutôt que minorer $\max \left\{\left|\Lambda_{i}\right|_{p_{0}} ; 1 \leq i \leq t\right\}$, nous allons minorer la norme de $u_{0}-$ $\lambda(u)$, norme relative à $k^{n} / W_{0}$ au plongement $\sigma_{0}$. Ceci est rendu possible par le résultat suivant :

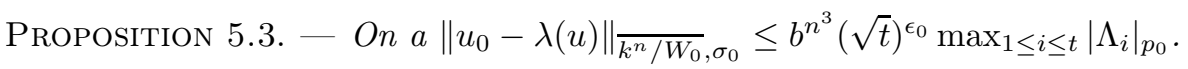

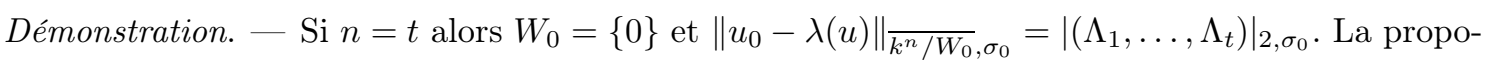
sition est vraie dans ce cas et, dans la suite, nous supposons $t \leq n-1$. Comme $\varphi\left(u_{0}-\lambda(u)\right)=$ $-\left(\Lambda_{1}, \ldots, \Lambda_{t}\right)$ on a

$$
\left\|u_{0}-\lambda(u)\right\|_{k^{n} / W_{0}}, \sigma_{0} \leq\left\|\varphi^{-1}\right\|_{\sigma_{0}}\left|\left(\Lambda_{1}, \ldots, \Lambda_{t}\right)\right|_{2, \sigma_{0}} \leq\left\|\varphi^{-1}\right\|_{\sigma_{0}}(\sqrt{t})^{\epsilon_{0}} \max _{1 \leq i \leq t}\left|\Lambda_{i}\right|_{p_{0}}
$$


où, si $\sigma$ est un plongement de $k,\left\|\varphi^{-1}\right\|_{\sigma}$ désigne la norme d'opérateur de $\varphi_{\sigma}^{-1}:\left(\mathbf{C}_{p}^{t},\left.|\cdot|\right|_{2, \sigma}\right) \rightarrow$ $\left(k^{n} / W_{0} \otimes_{\sigma} \mathbf{C}_{p},\|\cdot\|_{k^{n} / W_{0}}, \sigma\right)$. On a $\left\|\varphi^{-1}\right\|_{\sigma} \leq\|\varphi\|_{\sigma}^{t-1}\|\operatorname{det} \varphi\|_{\sigma}^{-1}$ (voir [G4, lemme 7.2] p. ex.) et $\|\operatorname{det} \varphi\|_{\sigma} \leq\|\varphi\|_{\sigma}^{t}$ par $1 \leq\|\varphi\|_{\sigma}\left\|\varphi^{-1}\right\|_{\sigma}$. On en déduit

$$
h\left(\overline{W_{0}}\right)=\widehat{\operatorname{deg}}_{\mathrm{n}} \overline{k^{n} / W_{0}}=h(\operatorname{det} \varphi) \leq \frac{t}{D} \sum_{\sigma} \log \max \left(1,\|\varphi\|_{\sigma}\right)-\frac{\log \left\|\varphi^{-1}\right\|_{\sigma_{0}}}{D} .
$$

De plus $h\left(\overline{W_{0}}\right) \geq 0$ car si $e_{i_{1}}, \ldots, e_{i_{t}}$ sont des vecteurs de la base canonique de $k^{n}$ dont les réductions $\overline{e_{i_{1}}}, \ldots, \overline{e_{i_{t}}}$ modulo $W_{0}$ forment une base de $k^{n} / W_{0}$, on a

$$
\begin{aligned}
& h\left(\overline{W_{0}}\right)=-\frac{1}{D} \sum_{p \in \mathcal{P}} \sum_{\sigma: k \hookrightarrow \mathbf{C}_{p}} \log \left\|\overline{e_{i_{1}}} \wedge \cdots \wedge \overline{e_{i_{t}}}\right\| \overline{\wedge^{t}\left(k^{n} / W_{0}\right)}, \sigma \\
& \geq-\frac{1}{D} \sum_{p \in \mathcal{P}} \sum_{\sigma: k \hookrightarrow \mathbf{C}_{p}} \log \left(\left\|\overline{e_{i_{1}}}\right\|_{\overline{k^{n} / W_{0}}, \sigma} \cdots\left\|\overline{e_{i_{t}}}\right\|_{\overline{k^{n} / W_{0}}, \sigma}\right) \\
& \geq-\frac{1}{D} \sum_{p \in \mathcal{P}} \sum_{\sigma: k \hookrightarrow \mathbf{C}_{p}} \log \left(\left|e_{i_{1}}\right|_{2, \sigma} \cdots\left|e_{i_{t}}\right|_{2, \sigma}\right)=0 .
\end{aligned}
$$

Par ailleurs la norme $\|\varphi\|_{\sigma}$ est inférieure à $\left|\left(\beta_{i, j}\right)_{i, j}\right|_{2, \sigma}$. On en déduit

$$
\begin{aligned}
\log \left\|\varphi^{-1}\right\|_{\sigma_{0}}^{1 / D} & \leq \frac{t}{D} \sum_{p \in \mathcal{P}} \sum_{\sigma: k \hookrightarrow \mathbf{C}_{p}} \log \max \left(1,\left|\left(\beta_{i, j}\right)_{i, j}\right|_{2, \sigma}\right) \\
& \leq t(\log \sqrt{n t}+n t) \frac{\log b}{D} .
\end{aligned}
$$

On conclut en majorant $t(\log \sqrt{n t}+n t)$ par $n^{3}$ comme nous l'avons fait à la fin de la proposition 5.2 , en tenant compte de $t \leq n-1$.

5.4. Choix des paramètres. - Soit $C_{0}:=(4 n)^{10 n}$. Posons $y:=0$ si $t=1$ et $\beta_{1,0} \neq 0$ et $y:=1$ sinon. Soit $S_{0}:=C_{0} \mathfrak{a}$ et $S:=C_{0}^{3} \mathfrak{a}$. Soit $U_{0}>0$ un nombre réel défini un peu plus loin (voir (13)). Soit $\widetilde{D}_{0}, \ldots, \widetilde{D}_{n}, \widetilde{T}, \widetilde{T}_{0}$ les nombres réels donnés par les formules suivantes :

$$
\begin{aligned}
\widetilde{T}_{0} & :=\frac{C_{0} U_{0}}{S \log \mathfrak{e}}, \quad \widetilde{T}:=C_{0}^{2} \widetilde{T}_{0}, \\
\widetilde{D}_{0} & :=\frac{U_{0}}{\log b+D \log S+S^{y} \log \mathfrak{e}}
\end{aligned}
$$

(la présence du paramètre $y$ au dénominateur explique le raffinement donné dans le théorème 5.1 lorsqu'il n'y a qu'une seule forme linéaire, non homogène) et

$$
\forall i \in\{1, \ldots, n\}, \quad \widetilde{D}_{i}:=\frac{U_{0}}{S \log \mathfrak{e}+D S \log a_{i}} .
$$

Notons $\bar{k}$ une clôture algébrique de $k$ dans $\mathbf{C}_{p_{0}}$. Un sous-groupe algébrique connexe $G^{\prime}$ de $G$ se décompose sur $\bar{k}$ en un produit $G_{0}^{\prime} \times \mathbf{G}_{\mathrm{m}}^{\prime}$ avec $G_{0}^{\prime}\left(\right.$ resp. $\left.\mathbf{G}_{\mathrm{m}}^{\prime}\right)$ un sous-groupe algébrique connexe de $G_{0}\left(\right.$ resp. $\left.\mathbf{G}_{\mathrm{m}}^{n}\right)$. Posons

$$
t^{\prime}:=\operatorname{dim} G_{0}^{\prime}, \quad n^{\prime}:=\operatorname{dim} \mathbf{G}_{\mathrm{m}}^{\prime}, \quad r^{\prime}:=\operatorname{codim}_{G} G^{\prime}, \quad \lambda^{\prime}:=\operatorname{codim}_{W}\left(W \cap t_{G^{\prime}}\right) .
$$

De simples considérations d'algèbre linéaire montrent que

$$
r^{\prime}-\lambda^{\prime}=t-\operatorname{dim}\left(t_{G_{0}^{\prime}}+\lambda\left(t_{\mathbf{G}_{\mathrm{m}}^{\prime}}\right)\right) \leq \min \left\{t-t^{\prime}, n-n^{\prime}\right\} .
$$

Par ailleurs, les groupes algébriques $G_{0}$ et $G$ admettent des compactifications naturelles $X_{0}:=$ $\mathbf{P}\left(k \oplus\left(k^{n} / W_{0}\right)^{v}\right)$ et $X:=X_{0} \times\left(\mathbf{P}_{k}^{1}\right)^{n}$. $\grave{A}$ une sous-variété $V$ de $X_{\bar{k}}$ est associé un polynôme dit de Hilbert-Samuel $H_{V}$ à $n+1$ variables (en prenant l'adhérence de Zariski de $V$ dans $X_{\bar{k}}$ ). Soit $\mathcal{H}\left(V ; X_{0}, \ldots, X_{n}\right)$ la partie homogène de plus haut degré de $H_{V}$ multipliée par $(\operatorname{dim} V)$ !. Les coefficients de $\mathcal{H}$ sont des entiers positifs de somme égale au degré de $V$. Par exemple, on a $\mathcal{H}\left(G ; X_{0}, \ldots, X_{n}\right)=\frac{(n+t) !}{t !} X_{0}^{t} X_{1} \cdots X_{n}$ (pour les propriétés de $\mathcal{H}$ nous renvoyons au texte de Roy [W3, chapitre 5]). Posons

$$
\mathrm{p}:=\left(u_{0}, \alpha_{1}, \ldots, \alpha_{n}\right) \quad \text { et } \quad \Sigma_{\mathrm{p}}(S):=\left\{0_{G}, \mathrm{p}, 2 \mathrm{p}, \ldots, S \mathrm{p}\right\}
$$


Lorsque $W+t_{G^{\prime}} \neq t_{G}$ (et en particulier $t^{\prime} \neq t$ ), on pose

$$
x\left(G^{\prime}\right):=\left(\frac{\widetilde{T}^{\lambda^{\prime}} \operatorname{card}\left(\frac{\Sigma_{\mathrm{p}}(S)+G^{\prime}\left(\mathbf{C}_{p_{0}}\right)}{G^{\prime}\left(\mathbf{C}_{p_{0}}\right)}\right) \mathcal{H}\left(G^{\prime} ; \widetilde{D}_{0}, \ldots, \widetilde{D}_{n}\right)}{C_{0} \mathcal{H}\left(G ; \widetilde{D}_{0}, \ldots, \widetilde{D}_{n}\right)}\right)^{\frac{1}{t-t^{\prime}}} .
$$

Soit $x$ le minimum des nombres réels $x\left(G^{\prime}\right)$ lorsque $G^{\prime}$ parcourt les sous-groupes algébriques connexes de $G$ tels que $W+t_{G^{\prime}} \neq t_{G}$ (l'existence de $x$ découle d'un argument standard, expliqué par exemple à la page 734 de $[\mathrm{G} 2]$ ). Soit $\widetilde{G}=\widetilde{G}_{0} \times \widetilde{\mathbf{G}}_{\mathrm{m}}$ un sous-groupe algébrique vérifiant ces conditions et tel que $x=x(\widetilde{G})$, auquel on adjoint les entiers $\widetilde{t}, \widetilde{n}, \widetilde{r}, \widetilde{\lambda}$ définis par (11). Nécessairement on a $\tilde{n}<n$ car sinon $t_{\widetilde{G}}+W=t_{G}$. Notons $T:=[\widetilde{T}], D_{0}:=\left[x \widetilde{D}_{0}\right]$ et, pour tout $i \in\{1, \ldots, n\}$, $D_{i}:=\left[\widetilde{D}_{i}\right]$. L'homogénéité de la fonction $\mathcal{H}$ permet de voir que la quantité $U_{0}^{\left(r^{\prime}-\lambda^{\prime}\right) /\left(t-t^{\prime}\right)} x\left(G^{\prime}\right)$ ne dépend pas de $U_{0}$, lorsque $G^{\prime}$ varie parmi les sous-groupes autorisés. En observant que $\{0\}$ fait partie de ces sous-groupes, nous pouvons choisir $U_{0}$ de sorte que $x(\{0\}) \leq 1$ (et, en particulier, on a $x \in] 0,1])$. Concrètement, la condition $x(\{0\}) \leq 1$ est satisfaite si l'on choisit $U_{0}$ égal à

$$
C_{0}^{(3 n-1) / t}\left\{\frac{t ! \operatorname{card}\left(\Sigma_{\mathbf{p}}(S)\right)}{(n+t) !} \prod_{i=1}^{n}\left(1+\frac{D \log a_{i}}{\log \mathfrak{e}}\right)\right\}^{1 / t} \times\left(\log b+D \log S+S^{y} \log \mathfrak{e}\right) .
$$

On notera que $\operatorname{card}\left(\Sigma_{\mathrm{p}}(S)\right)=S+1$ sauf, éventuellement, si toutes les conditions $p_{0}=\infty, n=1$, $\beta_{1,0}=0$ et $u_{1} \in \mathbf{Q} i \pi$ sont remplies. Mais ce cas est exclu par la réduction (iv) faite au début du $\S$ 5.2. On a donc $\operatorname{card}\left(\Sigma_{\mathrm{p}}(S)\right)=S+1$. L'expression (13) de $U_{0}$ permet de justifier les estimations suivantes.

Proposition 5.4. - Les propriétés suivantes sont satisfaites :

(i) $C_{0} \max \left\{1, \widetilde{D}_{0} / S^{1-y}, \widetilde{D}_{1}, \ldots, \widetilde{D}_{n}\right\} \leq \widetilde{T}_{0}$,

(ii) $D_{0} \neq 0$ et $C_{0} \leq \max _{1 \leq j \leq n}\left\{\widetilde{D}_{j}\right\}$ (en particulier l'un au moins des $D_{j}, 1 \leq j \leq n$, n'est pas nul),

(iii) $D \log \mathfrak{a} \leq 2 \mathfrak{a} \log \mathfrak{e}$,

(iv) $T \log \left(4 \widetilde{D}_{0}\right) \leq\left(10 n \log C_{0}\right) U_{0} / D$.

Démonstration. - Si les inégalités (i) et (ii) se vérifient aisément à partir des valeurs des paramètres, il n'est pas entièrement évident que $D_{0}$ est non nul, en raison du $x$ devant $\widetilde{D}_{0}$. Pour voir cela, on reprend l'argumentation du lemme 5.1, (iii), de G2 qui repose sur une propriété de décroissance d'un quotient de fonctions $\mathcal{H}$ et sur l'inégalité $\widetilde{\lambda} \geq n-\widetilde{n} \geq 1$. On a

$$
\begin{aligned}
\left(x \widetilde{D}_{0}\right)^{t-\widetilde{t}} & \geq \frac{\widetilde{T}^{\widetilde{\lambda}} \mathcal{H}\left(\widetilde{G} ; \widetilde{D}_{0}, \ldots, \widetilde{D}_{n}\right) \widetilde{D}_{0}^{t-\widetilde{t}}}{C_{0} \mathcal{H}\left(G ; \widetilde{D}_{0}, \ldots, \widetilde{D}_{n}\right)} \\
& \geq \frac{\widetilde{T}^{\tilde{\lambda}}}{C_{0}\left(\begin{array}{c}
n+t \\
t
\end{array}\right) \max _{1 \leq i \leq n}\left\{\widetilde{D}_{i}\right\}^{n-\widetilde{n}}} \\
& \geq \frac{C_{0}^{3 \widetilde{\lambda}-1}}{(n+t) !} \quad(\text { grâce à }(\mathrm{i})) .
\end{aligned}
$$

La valeur de $C_{0}$ entraîne alors $\left(x \widetilde{D}_{0}\right)^{t-\widetilde{t}} \geq 1$ puis $D_{0} \geq 1$. Venons-en maintenant à (iii) et (iv) qui requièrent aussi quelques détails. Tout d'abord, on a

$$
\log \mathfrak{a}=\log \left(\frac{\mathfrak{a} \log \mathfrak{e}}{D} \frac{D}{\log \mathfrak{e}}\right) \leq \frac{2 \mathfrak{a} \log \mathfrak{e}}{D}
$$

(car $\log x \leq x$ si $x \geq 1$ ), ce qui donne (iii). Par ailleurs, les définitions de $T$ et $\widetilde{D}_{0}$ combinées avec la valeur (13) de $U_{0}$ donnée ci-dessus impliquent

$$
T \log \left(4 \widetilde{D}_{0}\right) \leq \frac{U_{0}}{\mathfrak{a} \log \mathfrak{e}} \log \left(C_{0}^{3 n+3} \mathfrak{a} \prod_{i=1}^{n}\left(1+\frac{D \log a_{i}}{\log \mathfrak{e}}\right)\right) .
$$


En majorant le produit qui est dans le logarithme du majorant par

$$
\left(1+\frac{D}{\log \mathfrak{e}} \log \prod_{i=1}^{n} a_{i}\right)^{n} \leq\left(1+\exp \left\{\frac{2 \mathfrak{a} \log \mathfrak{e}}{D}\right\}\right)^{n}
$$

on a

$$
T \log \left(4 \widetilde{D}_{0}\right) \leq \frac{U_{0}}{\mathfrak{a} \log \mathfrak{e}}\left((3 n+3) \log C_{0}+\log \mathfrak{a}+3 n \frac{\mathfrak{a} \log \mathfrak{e}}{D}\right)
$$

On utilise alors (iii) pour majorer $\log \mathfrak{a}$ et conclure.

Désignons par $\Omega_{\sigma_{0}}$ l'ensemble $\{0\} \times(2 i \pi \mathbf{Z})^{n}$ si $\sigma_{0}$ est archimédien et l'ensemble $\{0\}$ sinon.

DÉfinition 5.5. - Nous dirons que nous sommes dans le cas périodique s'il existe un entier $m \in\{1, \ldots,(n+t) S\}$ tel que $m\left(u_{0}, u\right) \in t_{\widetilde{G}}\left(\mathbf{C}_{p_{0}}\right)+\Omega_{\sigma_{0}}$, et que nous sommes dans le cas non périodique dans le cas contraire.

Dans le cas périodique, la lettre $\Upsilon$ désigne l'ensemble des couples $(m, \tau)$ avec $m \in\{0,1, \ldots,(n+$ t) $S$ \} et $\tau=\left(\tau_{1}, \ldots, \tau_{n}\right) \in \mathbf{N}^{n}$ qui vérifie $\sum_{i=1}^{n} \tau_{i} \leq 2(n+t) T$ et $\tau_{n} \leq T_{0}$. Dans le cas non périodique, $\Upsilon$ est l'ensemble des couples $(m, \tau)$ avec $m \in\left\{0,1, \ldots, S_{0}-1\right\}$ et $\tau=\left(\tau_{1}, \ldots, \tau_{n}\right) \in \mathbf{N}^{n}$ qui vérifie $\sum_{i=1}^{n} \tau_{i} \leq 2(n+t) T$. Dans les deux cas, nous notons $\mu$ le cardinal de $\Upsilon$.

REMARQue 5.6. - Comme la famille $\left\{u_{1}, \ldots, u_{n}\right\}$ est libre sur $\mathbf{Q}$, être dans le cas périodique implique que le plongement $\sigma_{0}$ est archimédien et que la famille $\left\{2 i \pi, u_{1}, \ldots, u_{n}\right\}$ est liée sur $\mathbf{Q}$ (c'est-à-dire $\alpha_{1}, \ldots, \alpha_{n}$ multiplicativement dépendants). Comme il ne peut exister qu'une seule relation, à multiplication par un scalaire non nul près, entre $2 i \pi$ et les $u_{j}$ (sinon existerait une relation non triviale entre les $u_{j}$ ), le groupe $\widetilde{\mathbf{G}}_{\mathrm{m}}$ est de dimension $n-1$ et son espace tangent contient $W_{0}$.

5.5. Charnière de la démonstration et compléments au cas périodique. — Le groupe algébrique $\widetilde{\mathbf{G}}_{\mathrm{m}}$ est de dimension $\widetilde{n} \leq n-1$. En particulier, comme $\left\{u_{1}, \ldots, u_{n}\right\}$ est libre sur $\mathbf{Q}$ on a $u \notin t_{\widetilde{\mathbf{G}}_{\mathrm{m}}}\left(\mathbf{C}_{p_{0}}\right)$. Par conséquent les vecteurs $\left(u_{0}, u\right)$ et $(\lambda(u), u)$ n'appartiennent pas à l'espace tangent du groupe algébrique $\widetilde{G}\left(\mathbf{C}_{p_{0}}\right)$. L'intersection $W \cap t_{\widetilde{G}}$ est donc un sous-espace strict de $W$ (car ce dernier contient $(\lambda(u), u)$ ). Si $\sigma_{0}$ est archimédien, fixons alors une base orthonormée $w:=\left(w_{1}, \ldots, w_{n}\right)$ de $W \otimes_{\sigma_{0}} \mathbf{C}_{p_{0}}$ qui provient de $W \otimes_{\sigma_{0}} \mathbf{R}$ lorsque $\sigma_{0}$ est un plongement réel et qui possède en outre les deux propriétés suivantes :

(i) $\left(w_{1}, \ldots, w_{\mathfrak{n}}\right)$ est une base de $\left(W \cap t_{\widetilde{G}}\right) \otimes_{\sigma_{0}} \mathbf{C}_{p_{0}}$ (le $\mathfrak{n}$ gothique est la dimension de $\left.W \cap t_{\widetilde{G}}\right)$,

(ii) si $\mathfrak{u}:=\left(\mathfrak{u}_{1}, \ldots, \mathfrak{u}_{n}\right) \in \mathbf{C}_{p_{0}}^{n}$ désigne le vecteur des coordonnées de $(\lambda(u), u)$ dans la base $\left(w_{1}, \ldots, w_{n}\right)$ alors $\left|\mathfrak{u}_{n}\right|_{p_{0}}=\max \left\{\left|\mathfrak{u}_{j}\right|_{p_{0}} ; \mathfrak{n}+1 \leq j \leq n\right\}$.

Dans le cas périodique (et cette hypothèse implique maintenant que nécessairement $\sigma_{0}$ est archimédien puisque $\left.\left(u_{0}, u\right) \notin t_{\widetilde{G}}\left(\mathbf{C}_{p_{0}}\right)\right)$, l'on sait minorer $\left|\mathfrak{u}_{n}\right|_{p_{0}}$ de la manière suivante. Il existe un entier $m \in\{1, \ldots,(n+t) S\}, x \in t_{\widetilde{G}}(\mathbf{C})$ et $\omega \in(2 i \pi \mathbf{Z})^{n} \backslash\{0\}$ tels que $m\left(u_{0}, u\right)=x+(0, \omega)$. Soit $\mathrm{d}_{\sigma_{0}}$ la distance sur $t_{G}\left(\mathbf{C}_{p_{0}}\right)$ induite par la norme $\|\cdot\|_{t_{G}}, \sigma_{0}$. L'inégalité de Cauchy-Schwarz montre que $\left|\mathfrak{u}_{n}\right|_{p_{0}}$ est minoré par

$$
\begin{aligned}
\frac{\mathrm{d}_{\sigma_{0}}\left(m(\lambda(u), u), t_{\widetilde{G}}(\mathbf{C})\right)}{m \sqrt{n}} & =\frac{\mathrm{d}_{\sigma_{0}}\left(\left(m\left(\lambda(u)-u_{0}\right), \omega\right), t_{\widetilde{G}}(\mathbf{C})\right)}{m \sqrt{n}} \\
& \geq \frac{\mathrm{d}_{\sigma_{0}}\left(\omega, t_{\widetilde{\mathbf{G}}_{\mathrm{m}}}(\mathbf{C})\right)}{(n+t) S \sqrt{n}}
\end{aligned}
$$

À ce stade, nous aurons besoin d'un résultat que l'on trouve en substance dans $[\mathrm{BP}]$ et de manière plus explicite dans la démonstration de la proposition 6.1 de [Ré. Pour $x \in \mathbf{R}^{n}$, on note $|x|_{1}$ la somme des valeurs absolues des composantes de $x$.

LEMME 5.7. - Soit $H$ un sous-groupe algébrique connexe de $\mathbf{G}_{\mathrm{m}}^{n}$, de dimension $h \in\{1, \ldots, n-1\}$. Alors il existe une famille libre $\left\{\mathrm{h}_{i}:=\left(h_{i, 1}, \ldots, h_{i, n}\right) ; 1 \leq i \leq n-h\right\}$ de $\mathbf{Z}^{n}$ telle que

(i) $H=\left\{\left(x_{1}, \ldots, x_{n}\right) \in \mathbf{G}_{\mathrm{m}}^{n} ; \forall i \in\{1, \ldots, n-h\}, \prod_{j=1}^{n} x_{j}^{h_{i, j}}=1\right\}$

(ii) $\prod_{i=1}^{n-h}\left|\mathrm{~h}_{i}\right|_{1} \leq \frac{(n-h) !}{h !} \operatorname{deg} H$ (le degré est relatif au plongement usuel $\left.\mathbf{G}_{\mathrm{m}}^{n} \hookrightarrow\left(\mathbf{P}^{1}\right)^{n}\right)$. 
En utilisant ce lemme, nous allons minorer $\mathrm{d}_{\sigma_{0}}\left(\omega, t_{\widetilde{\mathbf{G}}_{\mathrm{m}}}(\mathbf{C})\right)$ de la manière suivante. Ce lemme appliqué à $H=\widetilde{\mathbf{G}}_{\mathrm{m}}$, qui est de dimension $n-1$ d'après la remarque 5.6. donne l'existence d'un vecteur $\mathrm{h}$ de $\mathbf{Z}^{n}$ qui forme une base de l'orthogonal de $t_{\widetilde{\mathbf{G}}_{\mathrm{m}}}(\mathbf{C})$ dans $\left(\mathbf{C}^{n},|\cdot|_{2, \sigma_{0}}\right)$ avec $|\mathrm{h}|_{1} \leq\left(\operatorname{deg} \widetilde{\mathbf{G}}_{\mathrm{m}}\right) /(n-1)$ !. Ainsi la projection $\omega^{\prime}$ de $\omega$ sur cet orthogonal est de norme égale à $\mathrm{d}_{\sigma_{0}}\left(\omega, t_{\widetilde{\mathbf{G}}_{\mathrm{m}}}(\mathbf{C})\right)$. Par Cauchy-Schwarz, le produit hermitien $\omega$.h est de valeur absolue inférieure à $|\mathrm{h}|_{2, \sigma_{0}}\left|\omega^{\prime}\right|_{2, \sigma_{0}}$. De plus ce produit est non nul car $\omega \notin t_{\widetilde{\mathbf{G}}_{\mathrm{m}}}(\mathbf{C})$ et il appartient à $2 i \pi \mathbf{Z}$. Comme $|\mathrm{h}|_{2, \sigma_{0}} \leq|\mathrm{h}|_{1}$, on en déduit l'inégalité

$$
\mathrm{d}_{\sigma_{0}}\left(\omega, t_{\widetilde{\mathbf{G}}_{\mathrm{m}}}(\mathbf{C})\right) \geq \frac{2 \pi(n-1) !}{\operatorname{deg} \widetilde{\mathbf{G}}_{\mathrm{m}}}
$$

puis

$$
\left|\mathfrak{u}_{n}\right| \geq\left(\frac{2 \pi(n-1) !}{(n+t) \sqrt{n}}\right) \frac{1}{S \operatorname{deg} \widetilde{\mathbf{G}}_{\mathrm{m}}} \geq\left(\frac{\pi(n-1) !}{n \sqrt{n}}\right) \frac{1}{S \operatorname{deg} \widetilde{\mathbf{G}}_{\mathrm{m}}}
$$

car $t \leq n$. On vérifie alors que le premier quotient à droite est supérieur à 1 ce qui conduit à l'énoncé suivant.

Proposition 5.8. - Dans le cas périodique, on a $\left|\mathfrak{u}_{n}\right|^{-1} \leq S \operatorname{deg} \widetilde{\mathbf{G}}_{\mathrm{m}}$.

Pour être utile, cette proposition devra être couplée avec le résultat suivant.

Proposition 5.9. - Dans le cas périodique, on a $\operatorname{deg} \widetilde{\mathbf{G}}_{\mathrm{m}} \leq C_{0}^{-2}\left(\begin{array}{c}n+t \\ t\end{array}\right) \widetilde{D}_{0}$.

Démonstration. - Par définition de $\widetilde{G}$, on a $x(\widetilde{G}) \leq x(\{0\}) \leq 1$ et la définition de $x(\widetilde{G})$ entraîne alors la majoration

$$
\frac{\widetilde{T}^{\tilde{\lambda}} \mathcal{H}\left(\widetilde{G} ; \widetilde{D}_{0}, \ldots, \widetilde{D}_{n}\right)}{C_{0} \mathcal{H}\left(G ; \widetilde{D}_{0}, \ldots, \widetilde{D}_{n}\right)} \leq 1 .
$$

En décomposant la fonction $\mathcal{H}$ sur les parties $G_{0}$ et $\mathbf{G}_{\mathrm{m}}^{n}$ et en utilisant la décroissance des fonctions partielles

$$
x_{i} \mapsto \frac{\mathcal{H}\left(\widetilde{G} ; \widetilde{D}_{0}, \ldots, x_{i}, \ldots, \widetilde{D}_{n}\right)}{\mathcal{H}\left(G ; \widetilde{D}_{0}, \ldots, x_{i}, \ldots, \widetilde{D}_{n}\right)}
$$

l'inégalité (14) devient

$$
\widetilde{T}^{\widetilde{\lambda}} \operatorname{deg} \widetilde{\mathbf{G}}_{\mathrm{m}} \leq C_{0}\left(\begin{array}{c}
n+t \\
t
\end{array}\right)\left(\widetilde{D}_{0}\right)^{t-\widetilde{t}} \max \left(\widetilde{D}_{1}, \ldots, \widetilde{D}_{n}\right)^{n-\widetilde{n}}
$$

D'après la remarque 5.6, on a $\operatorname{dim} \widetilde{\mathbf{G}}_{\mathrm{m}}=\widetilde{n}=n-1$. La formule (12) donne $\widetilde{\lambda}=\operatorname{dim}\left(t_{\widetilde{G}_{0}}+\lambda\left(t_{\widetilde{\mathbf{G}}_{\mathrm{m}}}\right)\right)-$ $\widetilde{t}+1$ et $t-\operatorname{dim}\left(t_{\widetilde{G}_{0}}+\lambda\left(t_{\widetilde{\mathbf{G}}_{\mathrm{m}}}\right)\right) \leq 1$. De (15) on tire alors la majoration

$$
\left(\frac{\widetilde{T}}{\max \left(\widetilde{D}_{0}, \ldots, \widetilde{D}_{n}\right)}\right)^{\tilde{\lambda}} \leq \frac{C_{0}\left(\begin{array}{c}
n+t \\
t
\end{array}\right) \widetilde{D}_{0}}{\operatorname{deg} \widetilde{\mathbf{G}}_{\mathrm{m}}}
$$

Si $y=1$ la proposition 5.9 découle de $\widetilde{T} \geq C_{0}^{3} \max \left(\widetilde{D}_{0}, \ldots, \widetilde{D}_{n}\right)$ (proposition 5.4, (i)). Si $y=0$ on a $t=1$ par définition de $y$ et, en utilisant $\widetilde{\lambda} \geq 1$ et $\widetilde{t} \geq 0$, l'inégalité (15) se simplifie en

$$
\frac{\widetilde{T}}{\max \left(\widetilde{D}_{1}, \ldots, \widetilde{D}_{n}\right)} \leq \frac{C_{0}\left(\begin{array}{c}
n+t \\
t
\end{array}\right) \widetilde{D}_{0}}{\operatorname{deg} \widetilde{\mathbf{G}}_{\mathrm{m}}}
$$

Le minorant est supérieur à $C_{0}^{3}$ et la proposition s'en déduit. 
5.6. Lemme de multiplicités. — Au $\S$ 3.7, nous avons introduit la notion de polynôme multihomogène. Elle va nous permettre de définir un espace vectoriel dans lequel sera la section auxiliaire que nous allons construire au $\S$ [5.10. Soit $E_{0}:=k \oplus\left(k^{n} / W_{0}\right)$ et, pour $i \in\{1, \ldots, n\}, E_{i}:=k \oplus k$. Posons

$$
E:=S^{D_{0}}\left(E_{0}^{\mathrm{v}}\right) \otimes_{k} \bigotimes_{i=1}^{n} S^{D_{i}}\left(E_{i}^{\mathrm{v}}\right) .
$$

Pour un plongement $\sigma: k \hookrightarrow \mathbf{C}_{p}$, soit $s \in E \otimes_{\sigma} \mathbf{C}_{p}$ et considérons $F_{s, \sigma}:\left(k^{n} / W_{0}\right) \otimes_{\sigma} \mathbf{C}_{p} \times \prod_{i=1}^{n} \mathcal{T}_{p} \rightarrow$ $\mathbf{C}_{p}$ l'application qui à $\left(z_{0}, z_{1}, \ldots, z_{n}\right)$ associe

$$
F_{s, \sigma}\left(z_{0}, z_{1}, \ldots, z_{n}\right)=s\left(\left(1, z_{0}\right),\left(1, e^{z_{1}}\right), \ldots,\left(1, e^{z_{n}}\right)\right)
$$

(dans cette écriture nous avons identifié $k$ et son dual via la base canonique).

DÉfinition 5.10. - Soit $\ell \in \mathbf{N}$ et $x=\left(x_{0}, x_{1}, \ldots, x_{n}\right) \in G\left(\mathbf{C}_{p}\right)$. On dit qu'un polynôme $s \in$ $E \otimes_{\sigma} \mathbf{C}_{p}$ s'annule au point $x$ à l'ordre $\ell$ le long de $W$ si l'application

$$
\left(z_{1}, \ldots, z_{n}\right) \mapsto s\left(\left(1, x_{0}+\lambda\left(z_{1}, \ldots, z_{n}\right)\right),\left(1, x_{1} e^{z_{1}}\right), \ldots,\left(1, x_{n} e^{z_{n}}\right)\right)
$$

s'annule à l'ordre $\ell$ en $(0, \ldots, 0), i$. e. si la série formelle en les variables $z_{1}, \ldots, z_{n}$ définie par cette application est un élément de l'idéal $\left(z_{1}, \ldots, z_{n}\right)^{\ell}$ de $\mathbf{C}_{p}\left[\left[z_{1}, \ldots, z_{n}\right]\right]$.

Si le point $x$ est l'image du vecteur $\left(\mathrm{z}_{0}, \mathbf{z}_{1}, \ldots, \mathbf{z}_{n}\right) \in\left(k^{n} / W_{0}\right) \otimes_{\sigma} \mathbf{C}_{p} \times \prod_{i=1}^{n} \mathcal{T}_{p}$ par l'application exponentielle de $G\left(\mathbf{C}_{p}\right)$ alors dire que $s$ s'annule au point $x$ à l'ordre $\ell$ le long de $W$ équivaut à dire que l'application

$$
\left(z_{1}, \ldots, z_{n}\right) \mapsto F_{s, \sigma}\left(\mathrm{z}_{0}+\lambda\left(z_{1}, \ldots, z_{n}\right), \mathrm{z}_{1}+z_{1}, \ldots, \mathrm{z}_{n}+z_{n}\right)
$$

s'annule à l'ordre $\ell$ au point $(0, \ldots, 0)$.

Passons maintenant au résultat principal de ce paragraphe.

Proposition 5.11. - Aucun élément non nul de $E \otimes_{\sigma} \mathbf{C}_{p}$ ne s'annule le long de $W$ à l'ordre $(n+t) T$ en tous les points de l'ensemble $\left\{0_{G}, \mathrm{p}, \ldots,(n+t) S \mathrm{p}\right\}$.

La démonstration est presque la même que celle de la proposition 5.3 de [G2, où le rôle joué par $\mathbf{G}_{\mathrm{m}}^{n}$ était dévolu à une variété abélienne. Ici, il y a quelques simplifications qui augmentent la clarté de la preuve. C'est la raison pour laquelle nous la reproduisons ci-après, sous une forme un peu abrégée.

Démonstration. - Supposons cet énoncé faux et qu'un tel polynôme existe. D'après le lemme de multiplicités $\left[\mathrm{Ph}\right.$, il existe un sous-groupe algébrique connexe $G_{0}^{\star}\left(\right.$ resp. $\left.\mathbf{G}_{\mathrm{m}}^{\star}\right)$ de $G_{0, \mathbf{C}_{p}}(r e s p$. de $\left.\mathbf{G}_{\mathrm{m}, \mathbf{C}_{p}}^{n}\right)$ tel que $G^{\star}:=G_{0}^{\star} \times \mathbf{G}_{\mathrm{m}}^{\star} \neq G_{\mathbf{C}_{p}}$ et

$$
T^{\lambda^{\star}} \operatorname{card}\left(\frac{\Sigma_{\mathrm{p}}(S)+G^{\star}\left(\mathbf{C}_{p}\right)}{G^{\star}\left(\mathbf{C}_{p}\right)}\right) \mathcal{H}\left(G^{\star} ; D_{0}^{\prime}, \ldots, D_{n}^{\prime}\right) \leq \mathcal{H}\left(G ; D_{0}^{\prime}, \ldots, D_{n}^{\prime}\right)
$$

où $D_{i}^{\prime}:=\max \left\{1, D_{i}\right\}$ pour tout $i \in\{1, \ldots, n\}$. Nous allons montrer que cette inégalité ne peut pas être satisfaite en distinguant deux cas. Quitte à permuter les facteurs dans $\mathbf{G}_{\mathrm{m}}^{n}$, l'on peut supposer $D_{1} \leq \cdots \leq D_{n}$ sans perte de généralité.

Premier cas : $t_{G^{\star}}+W \otimes_{\sigma} \mathbf{C}_{p}=t_{G_{\mathbf{C}_{p}}}$, c'est-à-dire $\lambda^{\star}=r^{\star}$. De l'inégalité (17) l'on déduit l'existence la majoration

$$
\operatorname{card}\left(\frac{\Sigma_{\mathrm{p}}(S)+G^{\star}\left(\mathbf{C}_{p}\right)}{G^{\star}\left(\mathbf{C}_{p}\right)}\right) \leq \frac{(n+t) !}{t !}\left(\frac{D_{0}}{T}\right)^{t-t^{\star}}\left(\frac{D_{n}}{T}\right)^{n-n^{\star}} .
$$

En observant que le cardinal à gauche vaut $S+1$ lorsque $t=1$ et $t^{\star}=0$, la première propriété de la proposition 5.4 contredit cette inégalité.

Second cas : $t_{G^{\star}}+W \otimes_{\sigma} \mathbf{C}_{p} \neq t_{G_{\mathbf{C}_{p}}}$. En particulier on a $t^{\star} \neq t$. Soit $\kappa$ le plus petit entier de $\{1, \ldots, n\}$ tel que $D_{\kappa} \geq 1$. Soit $\pi_{\kappa}: \mathbf{G}_{\mathrm{m}}^{n} \rightarrow \mathbf{G}_{\mathrm{m}}^{n-\kappa+1}$ la projection sur les $n-\kappa+1$ derniers facteurs. Posons $G_{\kappa}^{\star}:=G_{0}^{\star} \times \mathbf{G}_{\mathrm{m}}^{\kappa-1} \times \pi_{\kappa}\left(\mathbf{G}_{\mathrm{m}}^{\star}\right)$. Une propriété de la fonction $\mathcal{H}$ assure que

$$
\mathcal{H}\left(\pi_{\kappa}\left(\mathbf{G}_{\mathrm{m}}^{\star}\right) ; D_{\kappa}, \ldots, D_{n}\right) \leq \mathcal{H}\left(\mathbf{G}_{\mathrm{m}}^{\star} ; D_{1}, \ldots, D_{n}\right),
$$

ce qui entraîne

$$
\frac{\mathcal{H}\left(G ; D_{0}^{\prime}, \ldots, D_{n}^{\prime}\right)}{\mathcal{H}\left(G^{\star} ; D_{0}^{\prime}, \ldots, D_{n}^{\prime}\right)} \leq n ! \frac{\mathcal{H}\left(G ; \widetilde{D}_{0}, \ldots, \widetilde{D}_{n}\right)}{\mathcal{H}\left(G_{\kappa}^{\star} ; \widetilde{D}_{0}, \ldots, \widetilde{D}_{n}\right)} x^{t-t^{\star}}
$$


De plus, comme $G^{\star} \subseteq G_{\kappa}^{\star}$ on a $\lambda_{\kappa}^{\star}:=\operatorname{codim}_{W}\left(W \cap t_{G_{\kappa}^{\star}}\right) \leq \lambda^{\star}$ et

$$
\operatorname{card}\left(\frac{\Sigma_{\mathrm{p}}(S)+G_{\kappa}^{\star}\left(\mathbf{C}_{p}\right)}{G_{\kappa}^{\star}\left(\mathbf{C}_{p}\right)}\right) \leq \operatorname{card}\left(\frac{\Sigma_{\mathrm{p}}(S)+G^{\star}\left(\mathbf{C}_{p}\right)}{G^{\star}\left(\mathbf{C}_{p}\right)}\right) .
$$

De la sorte l'on obtient une inégalité analogue à (17) avec le sous-groupe algébrique $G_{\kappa}^{\star}$ :

$$
T^{\lambda_{\kappa}^{\star}} \operatorname{card}\left(\frac{\Sigma_{\mathrm{p}}(S)+G_{\kappa}^{\star}\left(\mathbf{C}_{p}\right)}{G_{\kappa}^{\star}\left(\mathbf{C}_{p}\right)}\right) \mathcal{H}\left(G_{\kappa}^{\star} ; \widetilde{D}_{0}, \ldots, \widetilde{D}_{n}\right) \leq n ! \mathcal{H}\left(G ; \widetilde{D}_{0}, \ldots, \widetilde{D}_{n}\right) x^{t-t^{\star}}
$$

En reprenant l'étude du premier cas (on a bien $G_{\kappa}^{\star} \neq G_{\mathbf{C}_{p}}$ car $t^{\star} \neq t$ ) et compte tenu du fait que $x \leq 1$, on montre que $\lambda_{\kappa}^{\star} \neq r_{\kappa}^{\star}$. Ceci signifie que $t_{G_{\kappa}^{\star}}+W \otimes_{\sigma} \mathbf{C}_{p} \neq t_{G_{\mathbf{C}_{p}}}$ et la majoration (18) entre alors en contradiction avec la définition de $x$.

5.7. Fibré adélique hermitien des sections auxiliaires. — Reprenons l'espace vectoriel $E$ défini au début du $\S 5.6$ et notons $\nu:=\operatorname{dim} E$ sa dimension. L'objectif de ce paragraphe est de munir $E$ d'une structure de fibré adélique hermitien assez particulière de manière à permettre d'extrapoler plus tard sur les dérivations. Tout d'abord, les espaces $E_{i}$ qui entrent dans la définition de $E$ sont naturellement munis d'une structure adélique hermitienne : pour $E_{0}=k \oplus k^{n} / W_{0}$ on choisit la structure quotient du fibré $\left(k^{n},|\cdot|_{2}\right)$ sur $k^{n} / W_{0}$ et l'on fait une somme directe hermitienne avec $k$ (muni de sa collection de valeurs absolues); pour $i \in\{1, \ldots, n\}$, on a $E_{i}=k \oplus k$ et l'on prend simplement la norme $|\cdot|_{2}$ de $k^{2}$. Comme nous l'avons décrit au $\S 3.7$ ces structures hermitiennes confèrent à $E$ une première structure de fibré adélique hermitien pur $\bar{E}:=\left(E,\left(\|\cdot\|_{\sigma}\right)_{\sigma}\right)$. Mais ce n'est pas tout à fait cette structure que l'on va mettre sur $E$. Pour les normes relatives au plongement $\sigma_{0}$ et à ses conjugués, nous allons ajouter une quantité supplémentaire, comme nous l'avons fait au $\S 4$ pour établir le lemme de Siegel approché absolu.

Normes particulières. — Soit $\left(e_{1}, \ldots, e_{n}\right)$ la base canonique de $k^{n}$. Si $\sigma_{0}$ est ultramétrique, la famille $w:=\left\{w_{i}:=\left(\lambda\left(e_{i}\right), e_{i}\right) ; 1 \leq i \leq n\right\}$ forme une base orthonormée de $W \otimes_{\sigma_{0}} \mathbf{C}_{p_{0}}$. En effet, pour tout $\mathbf{z}=\sum_{i=1}^{n} z_{i} e_{i} \in \mathbf{C}_{p_{0}}^{n}$, on a $z:=(\lambda(\mathbf{z}), \mathbf{z})=\sum_{i=1}^{n} z_{i} w_{i} \in W \otimes_{\sigma_{0}} \mathbf{C}_{p_{0}}$ et

$$
\|z\|_{\overline{t_{G}}, \sigma_{0}}=\max \left(\|\lambda(\mathbf{z})\|_{\overline{k^{n} / W_{0}}, \sigma_{0}},|z|_{2, \sigma_{0}}\right)=|z|_{2, \sigma_{0}}
$$

par propriété de la norme quotient. Dans le cas d'un plongement $\sigma_{0}$ archimédien, on note $\left(w_{1}, \ldots, w_{n}\right)$ la base orthonormée de $W \otimes_{\sigma_{0}} \mathbf{C}_{p_{0}}$ introduite au $\S$ [5.5. La famille $\left\{\left(\lambda\left(e_{i}\right), e_{i}\right) ; 1 \leq i \leq n\right\}$ est une base de $W$, dans laquelle les vecteurs $w_{i}$ peuvent s'écrire : il existe des formes linéaires $\left\{\mathrm{I}_{i}\left(z_{1}, \ldots, z_{n}\right) ; 1 \leq i \leq n\right\} \subseteq\left(\mathbf{C}_{p_{0}}^{n}\right)^{\vee}$ telles que

$$
\forall\left(z_{1}, \ldots, z_{n}\right) \in \mathbf{C}_{p_{0}}^{n}, \quad \sum_{i=1}^{n} z_{i} w_{i}=\sum_{i=1}^{n} \mathrm{I}_{i}\left(z_{1}, \ldots, z_{n}\right)\left(\lambda\left(e_{i}\right), e_{i}\right) .
$$

Les coefficients des $\mathrm{I}_{i}$ sont dans le complété $k_{\sigma_{0}}$ de $\sigma_{0}(k)$ dans $\mathbf{C}_{p_{0}}$ : c'est évident si $\sigma_{0}$ est complexe ou si $\sigma_{0}$ est ultramétrique car $\mathrm{I}_{i}\left(z_{1}, \ldots, z_{n}\right)=z_{i}$, et c'est aussi vrai si $\sigma_{0}$ est un plongement réel car dans ce cas $w_{i}$ a été choisi dans $W \otimes_{k} k_{\sigma_{0}}$. Pour $s \in E \otimes_{\sigma_{0}} \mathbf{C}_{p_{0}}, m \in \mathbf{N}$ et $\tau \in \mathbf{N}^{n}$, notons $\frac{1}{\tau !} \mathrm{D}_{w}^{\tau} F_{s, \sigma_{0}}\left(m\left(u_{0}, u\right)\right)$ le $\tau^{\text {ème }}$ coefficient de Taylor à l'origine de l'application

$$
z=\left(z_{1}, \ldots, z_{n}\right) \mapsto F_{s, \sigma_{0}}\left(m u_{0}+\lambda\left(\mathrm{I}_{1}(z), \ldots, \mathrm{I}_{n}(z)\right), m u_{1}+\mathrm{I}_{1}(z), \ldots, m u_{n}+\mathrm{I}_{n}(z)\right)
$$

(voir (16) pour la définition de $\left.F_{s, \sigma_{0}}\right)$. Soit $\left(s_{1}, \ldots, s_{\nu}\right)$ une base orthonormée de $\left(E \otimes_{k} k_{\sigma_{0}},\|\cdot\|_{\sigma_{0}}\right)$. Pour $z=\left(z_{0}, z_{1}, \ldots, z_{n}\right) \in t_{G_{0}}\left(\mathbf{C}_{p_{0}}\right) \oplus \mathcal{T}_{p_{0}}^{n}$, définissons

$$
\alpha_{\sigma_{0}}(z):=\prod_{j=1}^{n}\left|\left(1, e^{z_{j}}\right)\right|_{2, \sigma_{0}}^{-D_{j}} \times \operatorname{moy}_{\sigma_{0}}\left(1,\left\|z_{0}\right\|_{\overline{t_{G}}, \sigma_{0}}\right)^{-D_{0}} .
$$

Il existe $\alpha_{\sigma_{0}}^{\prime}\left(m\left(u_{0}, u\right)\right) \in k_{\sigma_{0}}$ de valeur absolue $p_{0}$-adique égale à $\alpha_{\sigma_{0}}\left(m\left(u_{0}, u\right)\right)$. C'est clair si $\sigma_{0}$ est complexe. Si $\sigma_{0}$ est ultramétrique, le vecteur $u_{0} \in k^{n} / W_{0}$ a des coordonnées dans $k_{\sigma_{0}}$ par rapport à une $k_{\sigma_{0}}$-base orthonormée de $\left(k^{n} / W_{0}\right) \otimes_{\sigma_{0}} k_{\sigma_{0}}$. Ainsi $\left\|u_{0}\right\|_{\overline{t_{G}}, \sigma_{0}}$, qui n'est rien d'autre que le maximum des valeurs absolues de ces coordonnées, est un élément de $\left|k_{\sigma_{0}}\right|_{p_{0}}$. C'est aussi le cas pour $\left|\left(1, \alpha_{j}^{m}\right)\right|_{2, \sigma_{0}}$ qui vaut $1=|1|_{p_{0}}$ ou $\left|\alpha_{j}^{m}\right|_{p_{0}}$. On a donc $\alpha_{\sigma_{0}}\left(m\left(u_{0}, u\right)\right) \in\left|k_{\sigma_{0}}\right|_{p_{0}}$ et l'existence 
de $\alpha_{\sigma_{0}}^{\prime}\left(m\left(u_{0}, u\right)\right)$ en découle. Considérons alors la matrice $\mathrm{A}_{\sigma_{0}}$, de taille $\mu \times \nu$, dont les coefficients sont les éléments de $k_{\sigma_{0}}$ suivants : pour tout $(m, \tau) \in \Upsilon$, pour tout $i \in\{1, \ldots, \nu\}$,

$$
\mathrm{A}_{\sigma_{0}}[(m, \tau), i]:=\left(\frac{1}{\tau !} \mathrm{D}_{w}^{\tau} F_{s_{i}, \sigma_{0}}\left(m\left(u_{0}, u\right)\right)\right) \times \alpha_{\sigma_{0}}^{\prime}\left(m\left(u_{0}, u\right)\right)
$$

Nous sommes maintenant en mesure de définir la norme voulue sur $E \otimes_{\sigma_{0}} \mathbf{C}_{p_{0}}$ : Soit $\alpha>0$ (choisi dans la proposition 5.17). Pour tout $s=\sum_{i=1}^{\nu} x_{i} s_{i} \in E \otimes_{\sigma_{0}} \mathbf{C}_{p_{0}}$,

$$
\|s\|_{E_{\alpha}, \sigma_{0}}:=\operatorname{moy}_{\sigma_{0}}\left(|x|_{2, \sigma_{0}}, \alpha\left|\mathrm{A}_{\sigma_{0}} x\right|_{2, \sigma_{0}}\right) .
$$

Dans ces expressions, $x$ désigne le vecteur colonne de coordonnées $x_{1}, \ldots, x_{\nu}$. Pour un plongement $\sigma$ conjugué à $\sigma_{0}$, c'est-à-dire définissant la même place $v_{0}$, nous choisissons la norme $\|\cdot\|_{E_{\alpha}, \sigma}$ de sorte que la collection de normes $\left(\|\cdot\|_{E_{\alpha}, \sigma}\right)_{\sigma \in v_{0}}$ soit invariante par Galois (au sens de la définition 3.1). En écrivant $\sigma=\iota \circ \sigma_{0}$ avec $\iota$ automorphisme continu de $\mathbf{C}_{p_{0}}$, on observe que $\|\cdot\|_{E_{\alpha}, \sigma}$ vérifie une égalité du type (22) en prenant $\mathrm{A}_{\sigma}=\iota\left(\mathrm{A}_{\sigma_{0}}\right)$ (les bases $\left(s_{1}, \ldots, s_{\nu}\right)$ et $w$ étant remplacées par leurs images par $\iota)$. Par définition, le fibré adélique hermitien $\bar{E}_{\alpha}$ est le fibré égal à $\bar{E}$ sauf pour les plongement $\sigma \in v_{0}$ où les normes sont données par $\left(\|\cdot\|_{\bar{E}_{\alpha}, \sigma}\right)_{\sigma \in v_{0}}$ (on retrouve $\bar{E}$ en prenant $\alpha=0)$.

LEMME 5.12. - La pente d'Arakelov normalisée $\widehat{\mu}(\bar{E})$ du fibré adélique hermitien $\bar{E}$ est minorée par $-D_{0} h\left(\overline{W_{0}}\right) /(t+1)$.

En réalité, on connaît une formule exacte pour cette pente, formule qui entraîne immédiatement le lemme. Pour cela, l'on peut se référer au lemme 7.3. de G4 en tenant compte du fait que la pente d'Arakelov est additive vis à vis du produit tensoriel de fibrés adéliques hermitiens (voir [G4, proposition 5.2]).

\subsection{Estimation du rang d'un système linéaire. -}

Lemme 5.13. - Soit $a, x, y$ des entiers naturels. Alors on a

$$
\left(\begin{array}{c}
x+y+a \\
a
\end{array}\right)-\left(\begin{array}{c}
x+a \\
a
\end{array}\right) \leq a y(x+y+1)^{a-1} .
$$

Démonstration. - $\mathrm{A} x$, $a$ fixés, la fonction $H: y \mapsto\left(\begin{array}{c}x+y+a \\ a\end{array}\right)-\left(\begin{array}{c}x+a \\ a\end{array}\right)$ est une fonction polynomiale à coefficients positifs. Cette fonction est convexe sur $\mathbf{R}^{+}$car de dérivée seconde positive. On a donc $H(y)=H(y)-H(0) \leq y H^{\prime}(y)$. Le calcul explicite de $H^{\prime}(y)$ comme une somme de $a$ produits de $a-1$ termes conduit à la majoration $H^{\prime}(y) \leq \sum_{i=1}^{a}(x+y+1)^{a-1}=a(x+y+1)^{a-1}$.

Proposition 5.14. - Soit $\rho$ le rang de la matrice $\mathrm{A}_{\sigma_{0}}$ de coefficients (21). Alors on a $\rho \leq$ $2(4 n)^{3 n} \nu / C_{0}$.

Démonstration. - Consubstantielle à la distinction entre le cas périodique et le cas non périodique et à l'introduction du groupe $\widetilde{G}$, l'argumentation n'a pas changé depuis l'article fondateur de Philippon \& Waldschmidt PW1]. En suivant l'approche plus effective de [Da, § 6.3] et en posant

$$
\left(T_{1}, S_{1}\right):= \begin{cases}\left(T, S_{0}\right) & \text { dans le cas non périodique } \\ \left(T_{0}, S\right) & \text { dans le cas périodique }\end{cases}
$$

on montre que

$$
\rho \leq X \operatorname{card}\left(\frac{\Sigma_{\mathrm{p}}\left(S_{1}\right)+\widetilde{G}(\bar{k})}{\widetilde{G}(\bar{k})}\right)\left(\operatorname{dim} \widetilde{G}+1+\mathcal{H}\left(\widetilde{G} ; D_{0}^{\prime}, \ldots, D_{n}^{\prime}\right)\right)
$$

avec

$$
X= \begin{cases}\left(\begin{array}{ll}
2(n+t) T+\widetilde{\lambda} \\
\widetilde{\lambda}
\end{array}\right) & \text { dans le cas non périodique } \\
\left(\begin{array}{c}
2(n+t) T+\widetilde{\lambda} \\
\widetilde{\lambda}
\end{array}\right)-\left(\begin{array}{c}
2(n+t) T-T_{0}+\widetilde{\lambda} \\
\widetilde{\lambda}
\end{array}\right) & \text { dans le cas périodique. }\end{cases}
$$


Le lemme 5.13 entraîne la majoration $X \leq(2(n+t))^{n} T^{\widetilde{\lambda}-1} T_{1}$. Par ailleurs, rappelons que, pour tout $i \in\{0, \ldots, n\}$, la fonction partielle $x_{i} \mapsto \frac{\mathcal{H}\left(\widetilde{G} ; x_{0}, \ldots, x_{n}\right)}{\mathcal{H}\left(G ; x_{0}, \ldots, x_{n}\right)}$ décroît sur $] 0,+\infty[$. Cette observation et les majorations $\widetilde{D}_{i} / 2 \leq D_{i}^{\prime}$ pour $i \in\{1, \ldots, n\}$ et $x \widetilde{D}_{0} / 2 \leq D_{0}$ impliquent

$$
\begin{aligned}
\frac{\mathcal{H}\left(\widetilde{G} ; D_{0}^{\prime}, \ldots, D_{n}^{\prime}\right)}{\mathcal{H}\left(G ; D_{0}^{\prime}, \ldots, D_{n}^{\prime}\right)} & \leq 2^{n+t} \frac{\mathcal{H}\left(\widetilde{G} ; x \widetilde{D}_{0}, \widetilde{D}_{1}, \ldots, \widetilde{D}_{n}\right)}{\mathcal{H}\left(G ; x \widetilde{D}_{0}, \ldots, \widetilde{D}_{n}\right)} \\
& =\frac{2^{n+t} C_{0}}{\widetilde{T}^{\widetilde{\lambda}} \operatorname{card}\left(\frac{\Sigma_{\mathrm{p}}(S)+\widetilde{G}(\bar{k})}{\widetilde{G}(\bar{k})}\right)}
\end{aligned}
$$

(l'égalité repose sur la définition de $x=x(\widetilde{G})$ ). En injectant cette estimation dans (23), nous avons

$$
\rho \leq 2^{n+t}(2(n+t))^{n+1} C_{0} \max \left\{\frac{T_{0}}{\widetilde{T}}, \frac{S_{0}}{S}\right\} \mathcal{H}\left(G ; D_{0}^{\prime}, \ldots, D_{n}^{\prime}\right) .
$$

La dimension $\nu$ de $E$ vaut $\left(\begin{array}{c}D_{0}+t \\ t\end{array}\right)\left(D_{1}+1\right) \cdots\left(D_{n}+1\right)$ et, en particulier, on a

$$
\mathcal{H}\left(G ; D_{0}^{\prime}, \ldots, D_{n}^{\prime}\right)=\frac{(n+t) !}{t !} D_{0}^{t} D_{1}^{\prime} \cdots D_{n}^{\prime} \leq(n+t) ! \nu
$$

Par conséquent, en majorant $(n+t) ! \operatorname{par}(n+t)^{n+t-1}$, on obtient

$$
\rho \leq 2(2(n+t))^{2 n+t} C_{0} \max \left\{\frac{T_{0}}{\widetilde{T}}, \frac{S_{0}}{S}\right\} \nu .
$$

Le choix des paramètres et l'inégalité $t \leq n$ permettent alors de conclure.

5.9. Estimation d'une dérivée. - Soit $\sigma: k \hookrightarrow \mathbf{C}_{p}$ un plongement de $k$. Soit $s \in E \otimes_{\sigma} \mathbf{C}_{p}$ que l'on écrit dans une base orthonormée comme dans (5) avec des coefficients $p_{i}$. On notera $L(s):=\sum_{i}\left|p_{i}\right|_{p}$ si $\sigma$ est archimédien et $L(s):=\max _{i}\left|p_{i}\right|_{p}$ si $\sigma$ est ultramétrique. Soit $\mathrm{w}=$ $\left(\mathrm{w}_{1}, \ldots, \mathrm{w}_{n}\right)$ une base de $W \otimes_{\sigma} \mathbf{C}_{p}$. En procédant de la même manière qu'au paragraphe 5.7 (en remplaçant la base $w$ d'alors par, ici, w), on dispose de la dérivée divisée $\frac{1}{\tau !} \mathrm{D}_{\mathrm{w}}^{\tau} F_{s, \sigma}(z)$ en un point $z=\left(z_{0}, \ldots, z_{n}\right) \in \mathbf{C}_{p}^{t} \times \mathcal{T}_{p}^{n}$ à l'ordre $\tau=\left(\tau_{1}, \ldots, \tau_{n}\right) \in \mathbf{N}^{n}$. Voici une première estimation, élémentaire malgré son aspect technique, d'un usage fréquent dans la suite.

Proposition 5.15. - Dans les conditions ci-dessus ( $\sigma, s, \mathrm{w}, z, \tau$ quelconques), on a

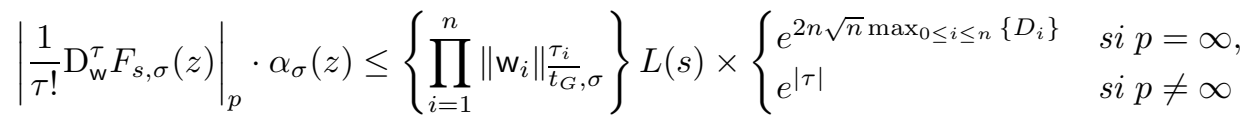

où

$$
\alpha_{\sigma}(z):=\prod_{j=1}^{n}\left|\left(1, e^{z_{j}}\right)\right|_{2, \sigma}^{-D_{j}} \times \operatorname{moy}_{\sigma}\left(1,\left\|z_{0}\right\|_{\overline{t_{G}}, \sigma}\right)^{-D_{0}} .
$$

Démonstration. - Tout d'abord, observons que si l'on multiplie le vecteur $\mathrm{w}_{i}$ par un nombre $\theta_{i} \in \mathbf{C}_{p}$ alors $\frac{1}{\tau !} \mathrm{D}_{\mathrm{w}}^{\tau} F_{s, \sigma}(z)$ est multiplié par $\prod_{i=1}^{n} \theta_{i}^{\tau_{i}}$. On peut donc supposer que, pour tout $i \in\{1, \ldots, n\},\left\|\mathrm{w}_{i}\right\|_{\overline{t_{G}}, \sigma}=1$. Considérons maintenant des formes linéaires $\mathrm{I}_{1}, \ldots, \mathrm{I}_{n} \in\left(\mathbf{C}_{p}^{n}\right)^{\mathrm{v}}$ telles que, pour tout $x=\left(x_{1}, \ldots, x_{n}\right) \in \mathbf{C}_{p}^{n}$, on a $\sum_{i=1}^{n} x_{i} \mathrm{w}_{i}=\sum_{i=1}^{n} \mathrm{l}_{i}(x)\left(\lambda\left(e_{i}\right), e_{i}\right)$ (rappelons que $e_{i}$ désigne le $i^{\text {ème }}$ vecteur de la base canonique de $\left.\mathbf{C}_{p}^{n}\right)$. La somme $\sum_{i=1}^{n} l_{i}\left(e_{j}\right) \lambda\left(e_{i}\right)$ est la composante de $\mathrm{w}_{j}$ sur $k^{n} / W_{0} \otimes_{\sigma} \mathbf{C}_{p}$. Elle est donc de norme plus petite que 1 .

Soit $\left(e_{0,1}, \ldots, e_{0, t}\right)$ la base orthonormée de $k^{n} / W_{0}$ choisie pour écrire les coefficients de $s$ (cf. (5) ). Le terme $\frac{1}{\tau !} \mathrm{D}_{\mathrm{w}}^{\tau} F_{s, \sigma}(z)$ que l'on cherche à évaluer est le coefficient devant $x^{\tau}$ de l'application

$$
x=\left(x_{1}, \ldots, x_{n}\right) \mapsto s\left(\left(1, z_{0}+\lambda\left(\mathrm{I}_{1}(x), \ldots, \mathrm{I}_{n}(x)\right)\right),\left(1, e^{z_{1}+\mathrm{I}_{1}(x)}\right), \ldots,\left(1, e^{z_{n}+\mathrm{I}_{n}(x)}\right)\right) .
$$

Celle-ci est une somme de termes de la forme

$$
p_{\mathrm{h}}\left\{\prod_{a=1}^{t}\left(e_{0, a}^{\mathrm{v}}\left(z_{0}\right)+\sum_{i=1}^{n} \mathrm{I}_{i}(x) e_{0, a}^{\mathrm{v}}\left(\lambda\left(e_{i}\right)\right)\right)^{\mathrm{h}_{0, a}}\right\} \prod_{i=1}^{n} e^{\mathrm{h}_{i} z_{i}+\mathrm{h}_{i} \mathrm{I}_{i}(x)}
$$

où $\mathrm{h}_{0}=\left(\mathrm{h}_{0,1}, \ldots, \mathrm{h}_{0, t}\right) \in \mathbf{N}^{t}, \mathrm{~h}=\left(\mathrm{h}_{0}, \mathrm{~h}_{1}, \ldots, \mathrm{h}_{n}\right) \in \mathbf{N}^{t} \times \mathbf{N}^{n}$ avec, pour tout $i \in\{0, \ldots, n\},\left|\mathrm{h}_{i}\right| \leq$ $D_{i}$, et $p_{\mathrm{h}} \in \mathbf{C}_{p}$ est l'un des coefficients de $s$. Comme seul le coefficient devant $x^{\tau}$ nous intéresse, 
on peut remplacer l'exponentielle $\exp \left\{\sum_{i=1}^{n} \mathrm{~h}_{i} \mathrm{l}_{i}(x)\right\}$ qui est dans (24) par son développement de Taylor à l'ordre $|\tau|$ :

$$
Q_{\mathrm{h}, \tau}(x):=\sum_{j=0}^{|\tau|} \frac{1}{j !}\left(\mathrm{h}_{1} \mathrm{l}_{1}(x)+\cdots+\mathrm{h}_{n} \mathrm{l}_{n}(x)\right)^{j} .
$$

De la sorte la quantité (24) peut être échangée par l'expression polynomiale

$$
p_{\mathrm{h}}\left\{\prod_{a=1}^{t}\left(e_{0, a}^{\mathrm{v}}\left(z_{0}\right)+\sum_{j=1}^{n} x_{j} e_{0, a}^{\mathrm{v}}\left(\sum_{i=1}^{n} \mathrm{l}_{i}\left(e_{j}\right) \lambda\left(e_{i}\right)\right)\right)^{\mathrm{h}_{0, a}}\right\}\left(\prod_{i=1}^{n} e^{\mathrm{h}_{i} z_{i}}\right) Q_{\mathrm{h}, \tau}(x) .
$$

La fonction longueur $\mathrm{P} \mapsto L(\mathrm{P})$ est sous-multiplicative : $L(\mathrm{PQ}) \leq L(\mathrm{P}) L(\mathrm{Q})$ (voir [W3, p. 76]). Cette propriété appliquée à la somme sur h des expressions (26) permet de majorer $\left|\frac{1}{\tau !} \mathrm{D}_{\mathrm{w}}^{\tau} F_{s, \sigma}(z)\right|_{p}$ par

$$
L(s)\left(\left\|z_{0}\right\|_{\overline{t_{G}}, \sigma}+n\right)^{D_{0}}\left\{\prod_{i=1}^{n} \max \left(1,\left|e^{z_{i}}\right|_{p}\right)^{D_{i}}\right\} \max _{\mathrm{h}}\left\{L\left(Q_{\mathrm{h}, \tau}\right)\right\}
$$

(on notera que le vecteur $e_{0, a}^{\mathrm{v}}$ est de norme 1 et donc $\left|e_{0, a}^{\mathrm{v}}(\mathrm{z})\right|_{p} \leq\|\mathrm{z}\|_{\overline{t_{G}}, \sigma}$ pour tout $\mathrm{z} \in k^{n} / W_{0} \otimes_{\sigma}$ $\mathbf{C}_{p}$ ). Pour conclure, il suffit d'estimer le maximum qui apparaît à la fin de cette expression. Lorsque le plongement $\sigma$ est archimédien, la longueur de $Q_{\mathrm{h}, \tau}$ est majorée par

$$
\sum_{j=0}^{|\tau|} \frac{1}{j !}\left(\sum_{i=1}^{n} D_{i}||_{i}(1, \ldots, 1) \mid\right)^{j}
$$

Or $\sum_{i=1}^{n} \mathrm{l}_{i}(1, \ldots, 1) e_{i}$ est la composante sur $\mathbf{C}_{p}^{n}$ de $\mathrm{w}_{1}+\cdots+\mathrm{w}_{n}$ et donc $\sum_{i=1}^{n}\left|\mathrm{I}_{i}(1, \ldots, 1)\right|^{2} \leq n^{2}$. Ainsi on a $\sum_{i=1}^{n} D_{i}||_{i}(1, \ldots, 1) \mid \leq \sqrt{D_{1}^{2}+\cdots+D_{n}^{2}} \times n \leq n \sqrt{n} \max \left\{D_{1}, \ldots, D_{n}\right\}$. On en déduit dans ce cas

$$
L\left(Q_{\mathrm{h}, \tau}\right) \leq e^{n \sqrt{n} \max \left\{D_{1}, \ldots, D_{n}\right\}} .
$$

Lorsque $\sigma$ est ultramétrique alors, pour tout $j \in \mathbf{N}$, on a $|j !|_{p}^{-1} \leq p^{j /(p-1)} \leq e^{j}$. Dans ce cas la longueur de $Q_{\mathrm{h}, \tau}$ est plus petite que $e^{|\tau|}$ et la proposition 5.15 est démontrée.

Corollaire 5.16. - Pour tout $\sigma \in v_{0}$, la norme d'opérateur de la matrice $\mathrm{A}_{\sigma}$, matrice définie au $\S$ [5.7, est inférieure à $e^{4 n U_{0}}$.

Démonstration. - Il suffit de le montrer pour $\sigma=\sigma_{0}$. On applique la proposition 5.15 à chacune des fonctions $F_{s_{i}, \sigma_{0}}$ qui intervient dans la définition (21) des coefficients de A $_{\sigma_{0}}$ avec $\sigma=\sigma_{0}$, $\mathrm{w}=w$ et $z=m\left(u_{0}, u\right)$. Supposons dans un premier temps que $\sigma_{0}$ est archimédien. D'après la fin du paragraphe 3.7, la longueur de $s_{i}$ est plus petite que

$$
\sqrt{t}^{D_{0}} \sqrt{2}^{D_{1}+\cdots+D_{n}} \leq\left(n 2^{n}\right)^{\max \left\{D_{0}, \ldots, D_{n}\right\} / 2} \leq e^{n \max \left\{D_{0}, \ldots, D_{n}\right\}} .
$$

Ainsi les coefficients de $\mathrm{A}_{\sigma_{0}}$ sont inférieurs à $e^{3 n \sqrt{n} \max _{0 \leq i \leq n}\left\{D_{i}\right\}}$, quantité elle-même plus petite que $e^{n U_{0}}$ d'après le choix des paramètres (début du $\S 5.4$ ) et car $\log C_{0} \geq 3 n$. En la comparant à la norme de Hilbert-Schmidt, on constate que la norme d'opérateur de $\mathrm{A}_{\sigma_{0}}$ est plus petite que $(\nu \mu)^{1 / 2} e^{n U_{0}}$. La dimension $\nu$ de $E$ vaut $\left(\begin{array}{c}D_{0}+t \\ t\end{array}\right)\left(D_{1}+1\right) \cdots\left(D_{n}+1\right)$ et donc

$$
\nu \leq\left(D_{0}+1\right)^{t}\left(D_{1}+1\right) \cdots\left(D_{n}+1\right) \leq e^{t D_{0}+D_{1}+\cdots+D_{n}} \leq e^{2 n \max \left\{D_{0}, \ldots, D_{n}\right\}} \leq e^{U_{0} / D} \leq e^{U_{0}}
$$

car le maximum des $\widetilde{D}_{i}$ est plus petit que $U_{0} /\left(D \log C_{0}\right)$. De même, $\mu$ est le cardinal de $\Upsilon$ (introduit à la suite de la définition 5.5. Il est inférieur à

$$
\begin{aligned}
\left(\begin{array}{c}
2(n+t) T+n \\
n
\end{array}\right) S(n+t) & \leq(2(n+t) T+1)^{n} S(n+t) \\
& \leq(4 n+1)^{n}(2 n) T^{n} S \\
& \leq \frac{(4 n+1)^{n}(2 n)(n+1) !}{C_{0}} \frac{U_{0}^{n+1}}{(n+1) !} \quad \text { car } S \leq U_{0} / C_{0} \\
& \leq 1 \times e^{U_{0}}=e^{U_{0}}
\end{aligned}
$$


Ainsi on trouve $\left\|\mathrm{A}_{\sigma_{0}}\right\|_{\sigma_{0}} \leq e^{(n+1) U_{0}} \leq e^{4 n U_{0}}$ lorsque $\sigma_{0}$ est archimédien. Dans le cas ultramétrique, la norme d'opérateur de $\mathrm{A}_{\sigma_{0}}$ est le maximum des coefficients de $\mathrm{A}_{\sigma_{0}}$ (voir proposition 3.15). D'après la proposition 5.15, on a $\left\|\mathrm{A}_{\sigma_{0}}\right\|_{\sigma_{0}} \leq e^{2(n+t) T} \leq e^{4 n U_{0}}$ car chacun des $s_{i}$ est de longueur 1 .

\subsection{Construction d'une section auxiliaire. -}

Proposition 5.17. - Soit $\alpha>0$ tel que $\log \alpha=C_{0}^{3 / 2} U_{0}$. Il existe une section $s \in E \otimes_{\sigma_{0}} \bar{k}$, non nulle, telle que

$$
h_{E_{\alpha}}(s) \leq \frac{\left[k_{\sigma_{0}}: \mathbf{Q}_{p_{0}}\right] \times(4 n)^{4 n} C_{0}^{1 / 2} U_{0}}{D} .
$$

Démonstration. - D'après le lemme de Siegel approché absolu du $\S$ 4 il existe un vecteur $s \in$ $E \otimes_{\sigma_{0}} \bar{k}$, non nul, tel que

$$
h_{\bar{E}_{\alpha}}(s) \leq \frac{\rho}{\nu D}\left(\sum_{\sigma \in v_{0}} \log \operatorname{moy}_{\sigma}(1, \alpha)+\log \max \left\{1,\left\|\mathrm{~A}_{\sigma}\right\|_{\sigma}\right\}\right)+\frac{1}{2} \log \nu-\widehat{\mu}(\bar{E})
$$

(on notera que le rang $\rho$ de $\mathrm{A}_{\sigma_{0}}$ est aussi celui de $\mathrm{A}_{\sigma}$ ). Dans le membre de droite, on majore $\rho / \nu$ avec la proposition 5.14 et $\left\|\mathrm{A}_{\sigma}\right\|_{\sigma}$ est évalué au moyen du corollaire 5.16 par $e^{4 n U_{0}}$. De plus, grâce au lemme 5.12 à la proposition 5.2 , au choix des paramètres $D_{0}$ et $x \leq 1$, on a

$$
-\widehat{\mu}(\bar{E}) \leq \frac{D_{0} h\left(\overline{W_{0}}\right)}{t+1} \leq \frac{n^{3} U_{0}}{D} .
$$

Enfin, on a $\frac{1}{2} \log \nu \leq U_{0} /(2 D)$ d'après (29). En remplaçant ces estimations dans (31), on trouve

$$
h_{\bar{E}_{\alpha}}(s) \leq \frac{2(4 n)^{3 n}\left[k_{\sigma_{0}}: \mathbf{Q}_{p_{0}}\right]}{D C_{0}} \times\left(\log \sqrt{2}+C_{0}^{3 / 2} U_{0}+4 n U_{0}\right)+\frac{U_{0}}{2 D}+\frac{n^{3} U_{0}}{D}
$$

et l'on conclut en utilisant la valeur de $C_{0}=(4 n)^{10 n}$.

La démonstration du théorème 5.1 s'effectue avec la section $s$ que l'on vient de construire dans cette proposition, qui vérifie (30). A priori cette section n'est pas définie sur $k$ mais sur une extension finie $K$ de $k$. Cette complication technique n'a pas de conséquence. En effet, le degré relatif $[K: k]$ n'intervient pas car les estimations des jets de $s$ en les plongements $\sigma^{\prime}$ de $K$ qui prolonge $\sigma: k \hookrightarrow \mathbf{C}_{p}$ sont de la forme $\mathfrak{c}_{\sigma}\|s\|_{\bar{E}_{\alpha}, \sigma^{\prime}}$, où $\mathfrak{c}_{\sigma}$ ne dépend que de $\sigma$ (et des autres données) et pas de $\sigma^{\prime}$. C'est la raison pour laquelle nous supposerons — et ceci sans perte de généralité — que $s$ est définie sur $k$.

5.11. Estimations générales. - Soit $(m, \ell) \in \mathbf{N}^{2}$. Considérons la section $s$ construite dans la proposition 5.17. Soit $\mathrm{w}=\left(\mathrm{w}_{1}, \ldots, \mathrm{w}_{n}\right)$ une $k$-base de $W$. Il existe des formes linéaires $\mathrm{I}_{1}, \ldots, \mathrm{I}_{n}$ sur $k^{n}$ telles que, si $x=\left(x_{1}, \ldots, x_{n}\right)$ est un $n$-uplet de variables, on ait

$$
\sum_{i=1}^{n} x_{i} \mathrm{w}_{i}=\left(\lambda\left(\mathrm{I}_{1}(x), \ldots, \mathrm{l}_{n}(x)\right), \mathrm{I}_{1}(x), \ldots, \mathrm{I}_{n}(x)\right) .
$$

Tous les coefficients de Taylor à l'origine - $a(s, \mathrm{w}, m, \tau), \tau \in \mathbf{N}^{n}$ - de la série formelle

$$
x=\left(x_{1}, \ldots, x_{n}\right) \mapsto s\left(\left(1, m u_{0}+\lambda\left(\mathrm{I}_{1}(x), \ldots, \mathrm{I}_{n}(x)\right)\right),\left(1, \alpha_{1}^{m} e^{\mathrm{I}_{1}(x)}\right), \ldots,\left(1, \alpha_{n}^{m} e^{\mathrm{I}_{n}(x)}\right)\right)
$$

sont des éléments de $k$. Étant donné un plongement $\sigma: k \hookrightarrow \mathbf{C}_{p}$ pour laquelle $m p$ possède un logarithme $z \in t_{G}\left(\mathbf{C}_{p}\right)$, l'image du coefficient $a(s, \mathrm{w}, m, \tau)$ dans $\sigma(k)$ est égale à $\frac{1}{\tau !} \mathrm{D}_{\mathrm{w}}^{\tau} F_{s, \sigma}(z)$ définie au $\S 5.7$

DÉFInition 5.18. - Soit $\left(\mathrm{w}_{1}^{\mathrm{v}}, \ldots, \mathrm{w}_{n}^{\mathrm{v}}\right)$ la base duale de w. Le jet de $s$ d'ordre $\ell$ le long de $W$ au point $m \mathrm{p}$, noté jet ${ }_{W}^{\ell} s(m \mathrm{p})$, est le vecteur

$$
\operatorname{jet}_{W}^{\ell} s(m \mathrm{p}):=\sum_{\substack{\tau=\left(\tau_{1}, \ldots, \tau_{n}\right) \in \mathrm{N}^{n} \\|\tau|=\ell}} a(s, \mathrm{w}, m, \tau) \cdot \prod_{i=1}^{n}\left(\mathrm{w}_{i}^{\mathrm{v}}\right)^{\tau_{i}}
$$

$\operatorname{de} S^{\ell}\left(W^{\mathrm{v}}\right)$ 
Tel que nous venons de le définir, le terme jet $_{W}^{\ell} s(m p)$ dépend du choix de la base w. Toutefois, lorsque jet ${ }_{W}^{h} s(m \mathrm{p})=0$ pour tout entier $h \in\{0, \ldots, \ell-1\}$, ce n'est plus le cas. Considérons le couple $(m, \ell) \in \mathbf{N}^{2}$ tel que $m \in\{0, \ldots,(n+t) S\}, \ell \in\{0, \ldots,(n+t) T\}$ et $(m, \ell)$ minimal pour la propriété jet ${ }_{W}^{\ell} s(m \mathrm{p}) \neq 0$. L'adjectif minimal s'entend par rapport à l'ordre lexicographique sur $\mathbf{N}^{2}$.

Seconde torsion de norme. — Il serait naturel (et possible) de travailler dans la suite avec $S^{\ell}\left(W^{\mathrm{v}}\right)$ muni de la structure de puissance symétrique définie au $\S 3.7$ Toutefois il s'avère plus pratique de modifier légèrement les normes sur $\overline{S^{\ell}\left(W^{\vee}\right)}$ de la manière suivante : pour un plongement $\sigma$ quelconque de $k$, considérons le coefficient $\alpha_{\sigma}\left(m\left(u_{0}, u\right)\right)$ introduit lors de la définition de $\mathrm{A}_{\sigma_{0}}$ (voir (20), p. 25). L'égalité $\alpha_{\iota \circ \sigma}\left(m\left(u_{0}, u\right)\right)=\alpha_{\sigma}\left(m\left(u_{0}, u\right)\right)$ pour tout automorphisme continu $\iota$ de $\mathbf{C}_{p}$ légitime la

DÉFInition 5.19. — Le fibré adélique hermitien $\overline{\mathfrak{J} \mathfrak{e t}}$ est le fibré sur $k$ d'espace sous-jacent $S^{\ell}\left(W^{\vee}\right)$ et sa norme en $\sigma: k \hookrightarrow \mathbf{C}_{p}$ est donnée par : pour tout $x \in S^{\ell}\left(W^{\vee}\right) \otimes_{\sigma} \mathbf{C}_{p}$, on a

$$
\|x\|_{\overline{\mathfrak{J e t}}, \sigma}:=\alpha_{\sigma}\left(m\left(u_{0}, u\right)\right)\|x\|_{\overline{S^{\ell}\left(W^{\vee}\right)}, \sigma} .
$$

Proposition 5.20. - La pente maximale de $\overline{\mathfrak{J} e \mathfrak{t}}$ est plus petite que $7 n^{2} U_{0} / D$.

Pour établir cette majoration, nous nous appuierons sur deux résultats auxiliaires.

Lemme 5.21. - La pente maximale de $\overline{S^{\ell}\left(W^{\vee}\right)}$ est inférieure à $2 n^{2} U_{0} / D$.

Démonstration. - Nous allons montrer que $\widehat{\mu}_{\max }\left(\overline{W^{v}}\right)$ est majoré par $(\log 2) / 2$. La proposition 3.16 et le fait que $\ell \leq(n+t) U_{0} / D$ permettront alors de conclure. L'application $k$-linéaire $\iota: k^{n} \rightarrow W, \iota(y):=(\lambda(y), y)$, est un isomorphisme d'espaces vectoriels. Les normes $\sigma$-adiques de l'application duale $\iota^{\vee}: W^{\vee} \rightarrow\left(k^{n}\right)^{\vee}$ sont plus petites que $(\sqrt{2})^{\epsilon_{\sigma}}$ avec $\epsilon_{\sigma}:=1$ si $\sigma$ est archimédien et 0 sinon. L'inégalité de pentes

$$
\widehat{\mu}_{\max }\left(\overline{W^{v}}\right) \leq \widehat{\mu}_{\max }\left(\left(k^{n},|\cdot|_{2}\right)^{\mathrm{v}}\right)+h\left(\iota^{\mathrm{v}}\right)
$$

(voir [G4, lemme 6.4]) entraîne le résultat voulu car la première quantité du majorant est nulle et l'autre inférieure à $(\log 2) / 2\left(\right.$ on notera que $\left.2 n((\log 2) / 2+2 \log n) \leq 2 n^{2}\right)$.

Lemme 5.22. - Pour tout entier $m$, la hauteur $h_{E_{0}}\left(1, m u_{0}\right)$ de $\left(1, m u_{0}\right)$ relative au fibré hermitien $\overline{E_{0}}=\overline{k \oplus\left(k^{n} / W_{0}\right)}$ est inférieure à $\log m+\left(2 n^{2} \log b\right) / D$.

Démonstration. - Dans cet énoncé, $u_{0}$ désigne la préimage de $-\left(\beta_{1,0}, \ldots, \beta_{t, 0}\right)$ par l'application $\varphi$ : $k^{n} / W_{0} \rightarrow k^{t}$, qui, à la classe d'équivalence de $z=\left(z_{1}, \ldots, z_{n}\right)$ modulo $W_{0}$, associe $\left(\ell_{1}(z), \ldots, \ell_{t}(z)\right)$ (voir $\S 5.3$, après la démonstration de la proposition 5.2). Ainsi, pour tout $z \in k^{n}$ tel que $\ell_{i}(z)=$ $-\beta_{i, 0}$ pour tout $i \in\{1, \ldots, t\}$, la norme de $u_{0}$ relative à $\overline{k^{n} / W_{0}}$ est inférieure à la norme de $z$ dans $\left(k^{n},|\cdot|_{2}\right)$. En particulier, on a $h_{\overline{E_{0}}}\left(1, m u_{0}\right) \leq h_{\left(k^{n+1},\left.\right|_{2}\right)}(1, m z)$. Au moyen d'une matrice extraite, il existe un ensemble $\mathrm{I} \subseteq\{1, \ldots, n\}$ à $t$ éléments, des déterminants $\left(\Delta_{\mathrm{i}}\right)_{\mathrm{i} \in \mathrm{I}}, \Delta$ de matrices à coefficients dans $\left\{ \pm \beta_{\mathrm{u}, \mathrm{v}} ; 0 \leq \mathrm{u} \leq t, 1 \leq \mathrm{v} \leq n\right\}$ et une solution $z$ du système $\ell_{i}(z)=-\beta_{i, 0}$, $1 \leq i \leq t$, telle que $z_{\mathrm{i}}=\Delta_{\mathrm{i}} / \Delta$ pour $\mathrm{i} \in \mathrm{I}$ et $z_{\mathrm{i}}=0$ sinon. On a alors

$$
h_{\left(k^{n+1},|\cdot|_{2}\right)}(1, m z)=h_{\left(k^{t+1},|\cdot|_{2}\right)}\left(\Delta, m\left(\Delta_{\mathrm{i}}\right)_{\mathrm{i} \in \mathrm{I}}\right) .
$$

Des majorations grossières des déterminants qui apparaissent ici conduisent à

$$
\left|\left(\Delta, m\left(\Delta_{\mathrm{i}}\right)_{\mathrm{i} \in \mathrm{I}}\right)\right|_{2, \sigma} \leq \prod_{\mathrm{u}, \mathrm{v}} \max \left\{1,\left|\beta_{\mathrm{u}, \mathrm{v}}\right|_{\sigma}\right\} \times \begin{cases}\left(1+m^{2} t\right)^{1 / 2} t ! & \text { si } p=\infty, \\ 1 & \text { si } p \neq \infty .\end{cases}
$$

Par conséquent, si $t \leq n-1$, on a

$$
h_{\left(k^{t+1},|\cdot|_{2}\right)}\left(\Delta, m\left(\Delta_{\mathrm{i}}\right)_{\mathrm{i} \in \mathrm{I}}\right) \leq(t+1) n \max _{\mathrm{u}, \mathrm{v}}\left\{h\left(\beta_{\mathrm{u}, \mathrm{v}}\right)\right\}+\log m+\log n !
$$

et la définition de $\log b$ permet de conclure. Si $t=n$ on a $W_{0}=\{0\}$ et nécessairement $z=u_{0}=$ $-\left(\beta_{1,0}, \ldots, \beta_{n, 0}\right)$. Dans ce cas, on a

$$
|(1, m z)|_{2, \sigma} \leq\left(1+m^{2} n\right)^{\epsilon_{\sigma} / 2} \prod_{i=1}^{n} \max \left(1,\left|\beta_{i, 0}\right|_{\sigma}\right)
$$


pour tout plongement $\sigma: k \hookrightarrow \mathbf{C}_{p}$ (rappelons que $\epsilon_{\sigma} \in\{0,1\}$ est nul si et seulement si $p \neq \infty$ ). On obtient alors

$$
h_{\left(k^{n+1},|\cdot|_{2}\right)}(1, m z) \leq \log m+\frac{\log (n+1)}{2}+\frac{n \log b}{D} .
$$

On conclut en utilisant $(\log b) / D \geq 1$ et $n+(\log (n+1)) / 2 \leq 2 n^{2}$.

Démonstration de la proposition [5.20. - Comme la pente maximale est un maximum, il existe un sous-espace $J \subseteq S^{\ell}\left(W^{\mathrm{v}}\right)$ tel que $\widehat{\mu}_{\max }(\overline{\mathfrak{J} \mathfrak{e t}})=\widehat{\mu}\left(J,\left(\|\cdot\|_{\overline{\mathfrak{J} \mathfrak{t}}, \sigma}\right)_{\sigma}\right)$, pente qui est elle-même égale

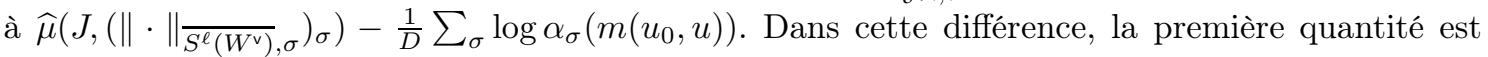
inférieure à $\widehat{\mu}_{\max }\left(\overline{S^{\ell}\left(W^{\vee}\right)}\right)$, qui a été estimée dans le lemme 5.21 ci-dessus. L'autre, avec le signe moins, est exactement égale à

$$
D_{0} h_{k \oplus\left(k^{n} / W_{0}\right)}\left(1, m u_{0}\right)+\sum_{j=1}^{n} D_{j} h_{\left(k^{2},|\cdot|_{2}\right)}\left(1, \alpha_{j}^{m}\right) .
$$

En vertu du lemme 5.22, le premier terme dans cette expression est majoré par $D_{0}(\log ((n+t) S)+$ $\left.\left(2 n^{2} \log b\right) / D\right) \leq 3 n^{2} U_{0} / D$. Par ailleurs, comme $\left(1+x^{2}\right)^{1 / 2} \leq \sqrt{2} \max \{1,|x|\}$, on a

$$
\begin{aligned}
h_{\left(k^{2},||_{2}\right)}\left(1, \alpha_{j}^{m}\right) & \leq \log \sqrt{2}+h\left(\alpha_{j}^{m}\right)=\log \sqrt{2}+m h\left(\alpha_{j}\right) \\
& \leq \log \sqrt{2}+(n+t) S \log a_{j}
\end{aligned}
$$

puis

$$
\begin{aligned}
\sum_{j=1}^{n} D_{j} h_{\left(k^{2},||_{2}\right)}\left(1, \alpha_{j}^{m}\right) & \leq\left(\sum_{j=1}^{n} D_{j}\right) \log \sqrt{2}+(n+t) \sum_{j=1}^{n} D_{j} S \log a_{j} \\
& \leq \frac{U_{0} n}{D} \log \sqrt{2}+n(2 n-1) \frac{U_{0}}{D} \\
& \leq \frac{2 n^{2} U_{0}}{D}
\end{aligned}
$$

En regroupant ces différentes majorations, on trouve

$$
\widehat{\mu}_{\max }(\overline{\mathfrak{J e t}}) \leq \frac{2 n^{2} U_{0}}{D}+\frac{3 n^{2} U_{0}}{D}+\frac{2 n^{2} U_{0}}{D}=\frac{7 n^{2} U_{0}}{D} .
$$

Dorénavant, la majeure partie de la démonstration du théorème 5.1 va consister à évaluer chacune des normes $\sigma$-adiques de jet ${ }_{W}^{\ell} s(m \mathrm{p})$ dans le but d'estimer sa hauteur, relative au fibré adélique hermitien $\overline{\mathfrak{J} \mathfrak{t}}$.

\subsubsection{Estimations archimédiennes. -}

Proposition 5.23. - Pour tout plongement complexe $\sigma: k \hookrightarrow \mathbf{C}$, on a

$$
\| \text { jet }_{W}^{\ell} s(m p)\left\|_{\mathfrak{J e t}, \sigma} \leq \exp \left\{n U_{0} / D\right\}\right\| s \|_{\bar{E}_{\alpha}, \sigma} .
$$

Démonstration. - Soit $\sigma: k \hookrightarrow$ C. Dans l'expression (33) du jet, choisissons pour w une base orthonormée de $W \otimes_{\sigma} \mathbf{C}$ et fixons un logarithme $m u$ de $m \mathrm{p} \in G\left(k_{\sigma}\right)$. La norme $\|$ jet $_{W}^{\ell} s(m \mathrm{p}) \|_{\overline{\mathfrak{J} \mathfrak{t} t}, \sigma}$ est majorée par

$$
\left(\begin{array}{c}
n+\ell-1 \\
\ell
\end{array}\right)^{1 / 2} \max _{|\tau|=\ell}\left\{\left|\frac{1}{\tau !} \mathrm{D}_{\mathrm{w}}^{\tau} F_{s, \sigma}(m u)\right|_{\sigma} \alpha_{\sigma}\left(m\left(u_{0}, u\right)\right)\right\} .
$$

La racine du facteur binomial est inférieure à

$$
(\ell+1)^{(n-1) / 2} \leq((n+t) T+1)^{(n-1) / 2}
$$

et donc à

$$
(2 n+1)^{(n-1) / 2}\left(\frac{U_{0}}{D}\right)^{(n-1) / 2} \leq(2 n+1)^{(n-1) / 2}(n-1) ! e^{\sqrt{U_{0} / D}} \leq e^{U_{0} /(2 D)}
$$


car $T \leq U_{0} / D$ et $x^{n-1} \leq(n-1) ! e^{x}$ pour $x \geq 0$. La proposition 5.15] majore la dérivée de $F_{s, \sigma}$ et l'on obtient alors

$$
\left\|\operatorname{jet}_{W}^{\ell} s(m \mathrm{p})\right\|_{\mathfrak{\mathfrak { J } e \mathfrak { t }}, \sigma} \leq e^{U_{0} /(2 D)} e^{2 n \sqrt{n} \max _{0 \leq i \leq n}\left\{D_{i}\right\}} L(s) .
$$

La longueur de $s$ qui apparaît ici peut être estimée comme à la fin du $\S$ 3.7. en utilisant (28), et l'on a $L(s) \leq\|s\|_{E_{\alpha}, \sigma} \exp \left\{n \max _{0 \leq i \leq n} D_{i}\right\}$. Le choix des paramètres montre que le maximum des $D_{i}$ est plus petit que $U_{0} /\left(D \log C_{0}\right)$, ce qui permet de conclure.

5.11.2. Estimations ultramétriques. - Étant donné des entiers $\ell$ et $h$ strictement positifs, on définit l'entier $\delta_{\ell}(h)$ comme le ppcm des produits $i_{1} \cdots i_{h^{\prime}}$ où $h^{\prime} \in\{1, \ldots, h\}, i_{j} \in \mathbf{N} \backslash\{0\}$ et $i_{1}+\cdots+i_{h^{\prime}} \leq \ell$. Le théorème des nombres premiers assure l'existence d'une constante absolue $c>0$ telle que $\log \delta_{\ell}(h) \leq \ell \log (c h)$. Au $\S 5.13$, nous utiliserons la valeur $c=4$ obtenue par Bruiltet dans $[\mathrm{Br}]$.

Proposition 5.24. - Pour tout plongement ultramétrique $\sigma: k \hookrightarrow \mathbf{C}_{p}$, pour tous entiers $m, \ell \geq$ 0 , pour toute section globale $s$ de $M$, le jet de $s$, d'ordre $\ell$ le long de $W$ au point mp, est de norme $\sigma$-adique inférieure à $\|s\|_{\bar{E}, \sigma} /\left|\delta_{\ell}\left(D_{0}\right)\right|_{p}$.

La démonstration est une variante plus simple de l'énoncé équivalent montré dans le cadre des variétés abéliennes au $\S 5.8$ de G2, au moyen du procédé de changement de variables de Chudnovsky. Nous renvoyons le lecteur à cet article pour plus de précisions.

5.12. Extrapolation sur les dérivations. - L'objectif de ce paragraphe est d'évaluer, pour tout plongement $\sigma$ conjugué à $\sigma_{0}$, la $\sigma$-norme $\operatorname{de~jet~}_{W}^{\ell} s(m \mathrm{p})$ en tenant compte de la construction de $s$. Comme les $\sigma \in v_{0}$ jouent tous le même rôle, il suffit d'établir les estimations pour $\sigma=\sigma_{0}$ (ce que nous supposons dorénavant). Comme de coutume, nous allons devoir distinguer les cas périodique et non périodique, distinction à laquelle se superposera la distinction entre $\sigma_{0}$ plongement complexe et $\sigma_{0}$ ultramétrique.

Soit $w$ la base particulière de $W \otimes_{\sigma_{0}} \mathbf{C}_{p_{0}}$ introduite au $\S$ [5.7, utilisée dans la définition de la matrice $\mathrm{A}_{\sigma_{0}}$. D'après la majoration (34) appliquée avec $\sigma=\sigma_{0}, \mathrm{w}=w$ et $z=m\left(u_{0}, u\right)$, le problème est d'estimer $\frac{1}{\tau !} \mathrm{D}_{w}^{\tau} F_{s, \sigma_{0}}\left(m\left(u_{0}, u\right)\right)$. Si $(m, \tau) \in \Upsilon$ alors la définition (21) des coefficients de la matrice $\mathrm{A}_{\sigma_{0}}$ implique

$$
\left|\frac{1}{\tau !} \mathrm{D}_{w}^{\tau} F_{s, \sigma_{0}}\left(m\left(u_{0}, u\right)\right)\right|_{p_{0}} \alpha_{\sigma_{0}}\left(m\left(u_{0}, u\right)\right) \leq \alpha^{-1}\|s\|_{E_{\alpha}, \sigma_{0}}
$$

et l'on obtient directement une estimation de $\frac{1}{\tau !} \mathrm{D}_{w}^{\tau} F_{s, \sigma_{0}}\left(m\left(u_{0}, u\right)\right)$, qui est meilleure que celle que l'on aura pour $(m, \tau) \notin \Upsilon$. C'est la raison pour laquelle nous supposerons maintenant que $(m, \tau) \notin \Upsilon$. On ramène alors le problème à $\frac{1}{\tau !} \mathrm{D}_{w}^{\tau} F_{s, \sigma_{0}}(m(\lambda(u), u))$ grâce au lemme de comparaison suivant :

Lemme 5.25. - Soit $\mathrm{w}=\left(\mathrm{w}_{1}, \ldots, \mathrm{w}_{n}\right)$ une base de $W \otimes_{\sigma_{0}} \mathbf{C}_{p_{0}}$. Pour tout $\mathrm{m} \in \mathbf{N}$ tel que $\| \mathrm{m}\left(u_{0}-\right.$ $\lambda(u)) \|_{t_{G}, \sigma_{0}} \leq 1$ et pour tout $\tau=\left(\tau_{1}, \ldots, \tau_{n}\right) \in \mathbf{N}^{n}$, de longueur $\leq 2(n+t) T$, la valeur absolue $p_{0}$-adique de la différence $\frac{1}{\tau !} \mathrm{D}_{\mathrm{w}}^{\tau} F_{s, \sigma_{0}}\left(\mathrm{~m}\left(u_{0}, u\right)\right)-\frac{1}{\tau !} \mathrm{D}_{\mathrm{w}}^{\tau} F_{s, \sigma_{0}}(\mathrm{~m}(\lambda(u), u))$ est majorée par

$$
e^{4 n U_{0}}\left\{\prod_{i=1}^{n}\left\|\mathrm{w}_{i}\right\| \|_{t_{G}}^{\tau_{i}}, \sigma_{0}\right\}\left\|\mathrm{m}\left(u_{0}-\lambda(u)\right)\right\|_{\bar{t}_{G}, \sigma_{0}} \alpha_{\sigma_{0}}\left(\mathrm{~m}\left(u_{0}, u\right)\right)^{-1}\|s\|_{E_{\alpha}, \sigma_{0}} .
$$

Démonstration. - Reprenons le début de la démonstration de la proposition 5.15 (avec $\sigma=\sigma_{0}$ ) et les formes linéaires $I_{1}, \ldots, l_{n}$ associées à la base $\mathrm{w}$ :

$$
\forall x=\left(x_{1}, \ldots, x_{n}\right) \in \mathbf{C}_{p_{0}}^{n}, \quad \sum_{i=1}^{n} x_{i} \mathrm{w}_{i}=\left(\lambda\left(\mathrm{I}_{1}(x), \ldots, \mathrm{I}_{n}(x)\right), \mathrm{I}_{1}(x), \ldots, \mathrm{I}_{n}(x)\right) .
$$

On peut supposer $\left\|\mathrm{w}_{i}\right\|_{\bar{t}_{G}, \sigma_{0}}=1$ pour tout $i \in\{1, \ldots, n\}$. Désignons par $\left(p_{\mathrm{h}}\right)_{\mathrm{h} \in \mathbf{N}^{t} \times \mathbf{N}^{n}}$ les coefficients de $s$ dans une écriture du type (5). La différence $\frac{1}{\tau !} \mathrm{D}_{\mathrm{w}}^{\tau} F_{s, \sigma_{0}}\left(\mathrm{~m}\left(u_{0}, u\right)\right)-\frac{1}{\tau !} \mathrm{D}_{\mathrm{w}}^{\tau} F_{s, \sigma_{0}}(\mathrm{~m}(\lambda(u), u))$ est 
la somme sur h des coefficients devant $x^{\tau}$ des fonctions

$$
\begin{aligned}
p_{\mathrm{h}} & \times\left(\prod_{i=1}^{n} \alpha_{i}^{\mathrm{mh}_{i}}\right) \times e^{\mathrm{h}_{1} \mathrm{I}_{1}(x)+\cdots+\mathrm{h}_{n} \mathrm{I}_{n}(x)} \\
\times & \left\{\prod_{a=1}^{t}\left(\mathrm{~m} e_{0, a}^{\mathrm{v}}\left(u_{0}\right)+\sum_{i=1}^{n} \mathrm{I}_{i}(x) e_{0, a}^{\mathrm{v}}\left(\lambda\left(e_{i}\right)\right)\right)^{\mathrm{h}_{0, a}}\right. \\
& \left.\quad-\prod_{a=1}^{t}\left(\mathrm{~m} e_{0, a}^{\mathrm{v}}(\lambda(u))+\sum_{i=1}^{n} \mathrm{I}_{i}(x) e_{0, a}^{\mathrm{v}}\left(\lambda\left(e_{i}\right)\right)\right)^{\mathrm{h}_{0, a}}\right\} .
\end{aligned}
$$

Dans cette expression, $\mathrm{h}_{0}=\left(\mathrm{h}_{0,1}, \ldots, \mathrm{h}_{0, t}\right) \in \mathbf{N}^{t}, \mathrm{~h}_{1}, \ldots, \mathrm{h}_{n} \in \mathbf{N}$ sont les coordonnées de $\mathrm{h}$. Comme $\mathrm{h}_{0}$ est de longueur $D_{0}$, la différence des quantités entre accolades est une expression du type

$$
\prod_{i=1}^{D_{0}}\left(a_{i}+b_{i}\right)-\prod_{i=1}^{D_{0}}\left(a_{i}^{\prime}+b_{i}\right)
$$

où $a_{i}, a_{i}^{\prime}$ sont les composantes de $\mathrm{m} u_{0}, \mathrm{~m} \lambda(u)$ respectivement dans la base orthonormée $e_{0}:=$ $\left(e_{0,1}, \ldots, e_{0, t}\right)$ et où $b_{i}$ est l'une des composantes de $\mathrm{I}(x):=\lambda\left(\mathrm{I}_{1}(x), \ldots, \mathrm{I}_{n}(x)\right)$ dans la base $e_{0}$ (répétée le bon nombre de fois pour avoir la puissance $\mathrm{h}_{0, a}$ dans (36)). Or la différence (37) est égale à

$$
\sum_{j=1}^{D_{0}}\left(a_{j}-a_{j}^{\prime}\right)\left(\prod_{i=1}^{j-1}\left(a_{i}^{\prime}+b_{i}\right)\right)\left(\prod_{i=j+1}^{D_{0}}\left(a_{i}+b_{i}\right)\right)
$$

(l'on écrit $a_{j}-a_{j}^{\prime}=\left(a_{j}+b_{j}\right)-\left(a_{j}^{\prime}+b_{j}\right)$ et l'on développe; les produits se simplifient deux à deux). Ainsi la différence des produits entre accolades dans (36), que l'on peut écrire symboliquement sous la forme

$$
\left(\mathrm{m} e_{0}^{\mathrm{v}}\left(u_{0}\right)+e_{0}^{\mathrm{v}}(\mathrm{I}(x))\right)^{\mathrm{h}_{0}}-\left(\mathrm{m} e_{0}^{\mathrm{v}}(\lambda(u))+e_{0}^{\mathrm{v}}(\mathrm{I}(x))\right)^{\mathrm{h}_{0}},
$$

est une somme de $D_{0}$ termes de la forme

$$
\delta\left(a, \mathrm{~h}_{0}\right)(x):=e_{0, a}^{\vee}\left(\mathrm{m}\left(u_{0}-\lambda(u)\right)\right) \times\left(\mathrm{m} e_{0}^{\vee}\left(u_{0}\right)+e_{0}^{\vee}(\mathrm{I}(x))\right)^{\mathrm{h}_{0}^{\prime}}\left(\mathrm{m} e_{0}^{\vee}(\lambda(u))+e_{0}^{\vee}(\mathrm{I}(x))\right)^{\mathrm{h}_{0}^{\prime \prime}}
$$

où $\mathrm{h}_{0}^{\prime}, \mathrm{h}_{0}^{\prime \prime}$ sont des vecteurs entiers (qui dépendent de $a$ ) dont la somme des longueurs vaut $D_{0}-1$. On notera que ce coefficient $\delta\left(a, \mathrm{~h}_{0}\right)$ est une fonction polynomiale de la variable $x$. La suite de la démonstration repose alors sur les mêmes considérations que celle de la proposition [5.15] Plus précisément, soit $Q_{\mathrm{h}, \tau}$ le polynôme de Taylor défini par (25). Le coefficient de $x^{\tau}$ de (36) est égal à celui du polynôme

$$
\sum_{D_{0} \text { termes }} p_{\mathrm{h}} \delta\left(a, \mathrm{~h}_{0}\right)(x)\left(\prod_{i=1}^{n} \alpha_{i}^{\mathrm{mh}_{i}}\right) Q_{\mathrm{h}, \tau}(x) .
$$

Ce coefficient est en valeur absolue plus petit que la longueur du polynôme. Ainsi la quantité $\left|\frac{1}{\tau !} \mathrm{D}_{\mathrm{w}}^{\tau} F_{s, \sigma_{0}}\left(\mathrm{~m}\left(u_{0}, u\right)\right)-\frac{1}{\tau !} \mathrm{D}_{\mathrm{w}}^{\tau} F_{s, \sigma_{0}}(\mathrm{~m}(\lambda(u), u))\right|_{p_{0}}$ est inférieure à

$$
\begin{aligned}
\| \mathrm{m} u_{0} & -\mathrm{m} \lambda(u) \|_{\overline{t_{G}}, \sigma_{0}} \times L(s) \max _{\mathrm{h}}\left\{L\left(Q_{\mathrm{h}, \tau}\right)\right\} \times \prod_{i=1}^{n} \max \left\{1,\left|\alpha_{i}\right|_{p_{0}}\right\}^{\mathrm{m} D_{i}} \\
& \times \begin{cases}D_{0}\left(\mathrm{~m}\left\|u_{0}\right\|_{\overline{t_{G}}, \sigma_{0}}+1+n\right)^{D_{0}-1} & \text { si } p_{0}=\infty \\
\max \left\{1,\left\|\mathrm{~m} u_{0}\right\|_{\overline{t_{G}}, \sigma_{0}}\right\}^{D_{0}-1} & \text { si } p_{0} \neq \infty\end{cases}
\end{aligned}
$$

(l'hypothèse $\left\|\mathrm{m}\left(u_{0}-\lambda(u)\right)\right\|_{\overline{t_{G}}, \sigma_{0}} \leq 1$ a permis ici de majorer la norme de $\mathrm{m} \lambda(u)$ et l'estimation de la longueur de I repose sur les estimations des sommes $\sum_{j=1}^{n} l_{i}\left(e_{j}\right) \lambda\left(e_{i}\right)$ introduites lors de la démonstration de la proposition [5.15). Pour conclure et si $\sigma_{0}$ est complexe, on majore $L(s)$ par $e^{U_{0}}\|s\|_{E_{\alpha}, \sigma_{0}}$ (fin du $\S\left[3.7\right.$ ), on majore $\max _{\mathrm{h}}\left\{L\left(Q_{\mathrm{h}, \tau}\right)\right\}$ par $e^{U_{0}}$ grâce à (27) et l'on majore $D_{0}\left(\mathrm{~m}\left\|u_{0}\right\|_{\bar{t}_{G}, \sigma_{0}}+1+n\right)^{D_{0}-1}$ par

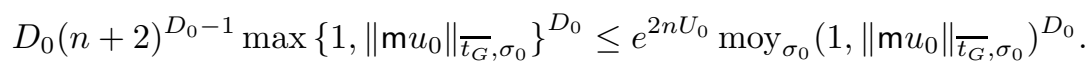

Si $\sigma_{0}$ est ultramétrique, on a $L(s)=\|s\|_{E_{\alpha}, \sigma_{0}}$ et $\max _{\mathrm{h}}\left\{L\left(Q_{\mathrm{h}, \tau}\right)\right\} \leq e^{|\tau|} \leq e^{2(n+t) T} \leq e^{4 n U_{0}}$. 
L'estimation de $\frac{1}{\tau !} \mathrm{D}_{w}^{\tau} F_{s, \sigma_{0}}(m(\lambda(u), u))$ repose sur une version légèrement affaiblie des lemmes d'interpolation de Waldschmidt W2] (cas archimédien) et de Roy [Ro] (cas ultramétrique, voir aussi [G3, lemme 4.21] pour les simplifications faites ici). Si $x$ est un nombre réel positif et $f$ une fonction définie sur le disque fermé $\bar{D}(0, x)=\left\{z \in \mathbf{C}_{p_{0}} ;|z|_{p_{0}} \leq x\right\}$, on note $|f|_{x}$ la borne supérieure des $|f(z)|, z \in \bar{D}(0, x)$. Rappelons que $\epsilon_{0}=0$ si $\sigma_{0}$ est ultramétrique et 1 sinon.

LeMme 5.26. - Soit $S_{1}, T_{1}$ des entiers naturels $\geq 2$ et $\mathrm{R}, \mathrm{r}$ des nombres réels vérifiant $\mathrm{R} \geq \mathrm{r} \geq$ $\left(2 S_{1}\right)^{\epsilon_{0}}$. Soit $f$ une fonction analytique dans le disque $\bar{D}(0, \mathrm{R})$. On a alors

$$
\begin{aligned}
|f|_{\mathrm{r}} \leq S_{1}^{T_{1}} \max & \left\{\left(\frac{\left(2 r_{p_{0}}\right)^{\epsilon_{0}} \mathrm{r}}{r_{p_{0}} \mathrm{R}}\right)^{T_{1} S_{1}}|f|_{\mathrm{R}},\right. \\
& \left.\left(\left(\frac{10 r_{p_{0}}}{S_{1}}\right)^{\epsilon_{0}} \frac{\mathrm{r}}{r_{p_{0}}}\right)^{T_{1} S_{1}} \max _{\substack{0 \leq h<T_{1} \\
0 \leq \mathrm{m}<S_{1}}}\left\{\left|\frac{1}{h !} f^{(h)}(\mathrm{m})\right|_{\sigma_{0}}\right\}\right\} .
\end{aligned}
$$

La suite de la démonstration repose sur un raisonnement par l'absurde, au moyen de l'hypothèse suivante.

НYPOTHËSE 5.27. $-\left\|u_{0}-\lambda(u)\right\|_{\overline{t_{G}}, \sigma_{0}} \leq \exp \left(-\left(C_{0}^{3 / 2}+4 n\right) U_{0}\right)$.

Notons D le disque ouvert $\left\{z \in \mathbf{C}_{p_{0}} ;|z|_{p_{0}}<\min \left\{r_{p_{0}} /\left|u_{i}\right|_{p_{0}} ; 1 \leq i \leq n\right\}\right\}$ si $\sigma_{0}$ est ultramétrique et l'ensemble $\mathbf{C}$ des nombres complexes sinon. On notera que les disques fermés, centrés en 0 et de rayons 1 et $\mathfrak{e} / r_{p_{0}}$ respectivement, sont tous les deux inclus dans $\mathbf{D}$.

5.12.1. Cas non périodique. - Soit $\tau \in \mathbf{N}^{n}$ de longueur $\ell$ (on rappelle que cet entier $\ell$, fixé après la définition [5.18, est inférieur à $(n+t) T)$. Soit $\mathrm{f}: \mathbf{D} \rightarrow \mathbf{C}_{p_{0}}$ l'application analytique définie par :

$$
\forall z \in \mathbf{D}, \quad \mathrm{f}(z)=\frac{1}{\tau !} \mathrm{D}_{w}^{\tau} F_{s, \sigma_{0}}(z(\lambda(u), u)) .
$$

Observons que, pour tout $h \in \mathbf{N}$ et tout $z \in \mathbf{D}$, on a

$$
\begin{aligned}
\frac{\mathrm{f}^{(h)}(z)}{h !} & =\frac{1}{\tau ! h !} \mathrm{D}_{w}^{\tau} \mathrm{D}_{(\lambda(u), u)}^{h} F_{s, \sigma_{0}}(z(\lambda(u), u)) \\
& =\sum_{\substack{j \in \mathbb{N}^{n} \\
|j|=h}}\left(\begin{array}{c}
\tau+j \\
\tau
\end{array}\right) \mathfrak{u}^{j} \frac{\mathrm{D}_{w}^{\tau+j}}{(\tau+j) !} F_{s, \sigma_{0}}(z(\lambda(u), u))
\end{aligned}
$$

$\left(\mathfrak{u}=\left(\mathfrak{u}_{1}, \ldots, \mathfrak{u}_{n}\right) \in \mathbf{C}_{p_{0}}^{n}\right.$ est le vecteur des coordonnées de $(\lambda(u), u)$ dans la base $\left.\left(w_{1}, \ldots, w_{n}\right)\right)$. Pour estimer $\frac{1}{\tau !} \mathrm{D}_{w}^{\tau} F_{s, \sigma_{0}}\left(m\left(u_{0}, u\right)\right)$, nous allons utiliser le lemme d'interpolation [5.26] afin de majorer $|\mathrm{f}(m)|_{p_{0}}$, avec les paramètres suivants :

(i) Si $\sigma_{0}$ est un plongement complexe alors $S_{1}:=S_{0}, \mathrm{r}:=2 m, \mathrm{R}:=2 \mathrm{re}, T_{1}:=(n+t) T$ et $f:=\mathrm{f}$.

(ii) Si $\sigma_{0}$ est un plongement ultramétrique alors $S_{1}:=S_{0}, \mathrm{r}:=1, \mathrm{R}:=\mathfrak{e} / r_{p_{0}}, T_{1}:=(n+t) T$ et $f:=\mathrm{f}$.

Avec ces choix, la proposition [5.15] fournit l'estimation suivante :

Proposition 5.28. - On a $\alpha_{\sigma_{0}}\left(m\left(u_{0}, u\right)\right)|\mathrm{f}|_{\mathrm{R}} \leq \exp \left\{17 n^{2} U_{0}\right\}\|s\|_{\bar{E}_{\alpha}, \sigma_{0}}$.

Démonstration. - En remarque liminaire, mentionnons que les majorations qui suivent sont volontairement très larges, afin de rester valide dans le cas périodique, qui sera étudié au prochain paragraphe.

D'après la proposition [5.15, on a

$$
|\mathrm{f}(z)|_{p_{0}} \cdot \alpha_{\sigma_{0}}(z(\lambda(u), u)) \leq L(s) \times \begin{cases}e^{2 n \sqrt{n} \max _{0 \leq j \leq n} D_{j}} & \text { si } p_{0}=\infty \\ e^{4 n T} & \text { si } p_{0} \neq \infty .\end{cases}
$$

Comme nous l'avons déjà vu (voir fin de la démonstration de la proposition 5.23), la longueur de $s$ est plus petite que $e^{n \max _{0 \leq j \leq n} D_{j}}\|s\|_{\bar{E}_{\alpha}, \sigma_{0}}$ si $p_{0}=\infty$ et $L(s) \leq\|s\|_{\bar{E}_{\alpha}, \sigma_{0}}$ si $p_{0} \neq \infty$. Le choix des paramètres donne alors

$$
|\mathrm{f}(z)|_{p_{0}} \cdot \alpha_{\sigma_{0}}(z(\lambda(u), u)) \leq e^{4 n U_{0}}\|s\|_{\bar{E}_{\alpha}, \sigma_{0}} .
$$


En utilisant la définition (20) de $\alpha_{\sigma_{0}}$, la quantité $\alpha_{\sigma_{0}}(z(\lambda(u), u))^{-1}$ est, pour $|z|_{p_{0}} \leq \mathrm{R}$, majorée par

$$
\begin{cases}\left(1+64\left(n S \mathfrak{e}\|\lambda(u)\|_{\overline{t_{G}}, \sigma_{0}}\right)^{2}\right)^{D_{0} / 2} \prod_{j=1}^{n}\left(1+e^{16 n S \mathfrak{e}\left|u_{j}\right|_{p_{0}}}\right)^{D_{j} / 2} & \text { si } p_{0}=\infty, \\ \max \left\{1,2 \mathfrak{e}\|\lambda(u)\|_{\bar{t}_{G}, \sigma_{0}}\right\}^{D_{0}} & \text { si } p_{0} \neq \infty\end{cases}
$$

(dans le deuxième cas, nous avons majoré $r_{p_{0}}^{-1}=p_{0}^{\frac{1}{p_{0}-1}}$ par 2). Dans le cas ultramétrique l'hypothèse 5.27 implique $\|\lambda(u)\|_{\bar{t}_{G}, \sigma_{0}}=\left\|u_{0}\right\|_{\bar{t}_{G}, \sigma_{0}}$ et

$$
\max \left\{1,2 \mathfrak{e}\left\|u_{0}\right\|_{\overline{t_{G}}, \sigma_{0}}\right\}^{D_{0}} \leq(2 \mathfrak{e})^{D_{0}} \max \left\{1,\left\|u_{0}\right\|_{\overline{t_{G}}, \sigma_{0}}\right\}^{D_{0}} .
$$

On trouve ainsi

$$
\frac{\alpha_{\sigma_{0}}(z(\lambda(u), u))^{-1}}{\alpha_{\sigma_{0}}\left(m\left(u_{0}, u\right)\right)^{-1}} \leq(2 \mathfrak{e})^{D_{0}} \leq e^{2 U_{0}} .
$$

Le résultat est alors acquis car $2+4 n \leq 17 n^{2}$. Dans le cas archimédien, on majore $\|\lambda(u)\|_{t_{G}, \sigma_{0}}^{2}$ par $2\left(\left\|\lambda(u)-u_{0}\right\|_{t_{G}, \sigma_{0}}^{2}+\left\|u_{0}\right\| \frac{2}{t_{G}, \sigma_{0}}\right)$. Le quotient

$$
\frac{\alpha_{\sigma_{0}}(z(\lambda(u), u))^{-1}}{\alpha_{\sigma_{0}}\left(m\left(u_{0}, u\right)\right)^{-1}}
$$

est majoré par

$$
\sqrt{2}^{\sum_{j=0}^{n} D_{j}}(8 n \mathfrak{e} S)^{D_{0}}\left(1+\left\|u_{0}\right\|_{t_{G}}^{2}, \sigma_{0}\right)^{D_{0} / 2} \exp \left\{8 n S \mathfrak{e}\left(\sum_{j=1}^{n} D_{j}\left|u_{j}\right|_{p_{0}}\right)\right\} .
$$

Le choix des paramètres entraîne

(i) $8 n S \mathfrak{e}\left(\sum_{j=1}^{n} D_{j}\left|u_{j}\right|_{p_{0}}\right) \leq 8 n^{2} U_{0}$

(ii) $\sqrt{2}^{\sum_{j=0}^{n} D_{j}}(8 n \mathfrak{e} S)^{D_{0}} \leq e^{3 U_{0}}$,

(iii) $\operatorname{moy}_{\sigma_{0}}\left(1,\left\|u_{0}\right\|_{\bar{t}_{G}, \sigma_{0}}\right)^{D_{0}} \leq \exp \left(D D_{0} h_{\overline{E_{0}}}\left(1, u_{0}\right)\right) \leq e^{2 n^{2} U_{0}}$ (lemme 5.22 ).

On trouve ainsi

$$
\frac{\alpha_{\sigma_{0}}(z(\lambda(u), u))^{-1}}{\alpha_{\sigma_{0}}\left(m\left(u_{0}, u\right)\right)^{-1}} \leq e^{\left(10 n^{2}+3\right) U_{0}} \quad\left(\text { pour }|z|_{p_{0}} \leq \mathrm{R}\right)
$$

et l'on conclut avec $10 n^{2}+3+4 n \leq 17 n^{2}$.

Par ailleurs, au moyen de la formule (40), du lemme 5.25 et de la construction de la section $s$, on a

$$
\max _{\substack{0 \leq h<T_{1} \\ 0 \leq \mathrm{m}<S_{1}}}\left\{\left|\frac{1}{h !} \mathrm{f}^{(h)}(\mathrm{m})\right|_{p_{0}}\right\} \leq \theta_{0}\left(e^{4 n U_{0}}\left\|u_{0}-\lambda(u)\right\|_{\bar{t}_{G}, \sigma_{0}}+\alpha^{-1}\right)\|s\|_{\bar{E}_{\alpha}, \sigma_{0}}
$$

où

$$
\theta_{0}:=\max _{0 \leq \mathrm{m}<S_{0}}\left\{\alpha_{\sigma_{0}}\left(\mathrm{~m}\left(u_{0}, u\right)\right)^{-1}\right\} \times \begin{cases}\left(n+\sum_{j=1}^{n}\left|\mathfrak{u}_{j}\right|_{p_{0}}\right)^{2(n+t) T} & \text { si } p_{0}=\infty, \\ 1 & \text { si } p_{0} \neq \infty .\end{cases}
$$

La somme des $\left|\mathfrak{u}_{j}\right|_{p_{0}}$ est majorée par $\sqrt{n}\|(\lambda(u), u)\|_{\overline{t_{G}}, \sigma_{0}}$, lui-même plus petit que $\sqrt{2 n}|u|_{2, \sigma_{0}}$. On a $|u|_{2, \sigma_{0}} \leq \sum_{j=1}^{n}\left|u_{j}\right|_{p_{0}}$ et avec la définition de $\log a_{j}$ on trouve

$$
\begin{aligned}
n+\sum_{j=1}^{n}\left|\mathfrak{u}_{j}\right|_{p_{0}} & \leq n+\sqrt{2 n}\left(\frac{D}{\mathfrak{e}}\right) \log \prod_{j=1}^{n} a_{j} \\
& \leq n+\sqrt{2 n} \exp \{2 \mathfrak{a} \log \mathfrak{e}\} .
\end{aligned}
$$

En posant $\mathfrak{x}:=\mathfrak{a} \log \mathfrak{e} \geq 1$, on a

$$
\left(n+\sqrt{2 n} e^{2 \mathfrak{x}}\right)^{4 n / \mathfrak{x}} \leq e^{8 n}\left(n e^{-2}+\sqrt{2 n}\right)^{4 n} \leq e^{10 n^{2}} .
$$

La majoration $T \leq U_{0} /(\mathfrak{a} \log \mathfrak{e})$ entraîne alors

$$
\theta_{0} \leq \max _{0 \leq \mathrm{m}<S_{1}}\left\{\alpha_{\sigma_{0}}\left(\mathrm{~m}\left(u_{0}, u\right)\right)^{-1}\right\} \exp \left\{10 n^{2} U_{0}\right\}
$$


En procédant de la même manière que pour la majoration (41), on a, pour tout entier m entre 1 et $S_{1}$

$$
\begin{aligned}
\alpha_{\sigma_{0}}\left(\mathrm{~m}\left(u_{0}, u\right)\right)^{-1} & \leq(2 n S)^{D_{0}} e^{2 n^{2} U_{0}} \prod_{j=1}^{n}\left(\sqrt{2} e^{D \mathrm{~m} \log a_{j}}\right)^{D_{j}} \\
& \leq \exp \left(\left(1+\frac{\log (2 n)}{\log C_{0}}+2 n^{2}+\frac{n \log \sqrt{2}}{C_{0}}+n(n+t)\right) U_{0}\right) \\
& \leq e^{5 n^{2} U_{0}}
\end{aligned}
$$

Comme $\alpha_{\sigma_{0}}\left(m\left(u_{0}, u\right)\right) \leq 1$, on en déduit

$$
\alpha_{\sigma_{0}}\left(m\left(u_{0}, u\right)\right) \cdot \max _{0 \leq \mathrm{m}<S_{1}}\left\{\alpha_{\sigma_{0}}\left(\mathrm{~m}\left(u_{0}, u\right)\right)^{-1}\right\} \leq e^{5 n^{2} U_{0}} .
$$

De plus $\log \alpha$ a été choisi supérieur à $C_{0}^{3 / 2} U_{0}$ (proposition [5.17) et $\left\|u_{0}-\lambda(u)\right\|_{\overline{t_{G}}, \sigma_{0}}$ est plus petit que $e^{-\left(C_{0}^{3 / 2}+4 n\right) U_{0}}$ d'après l'hypothèse 5.27 . Les calculs ci-dessus montrent alors que

$$
\begin{aligned}
\alpha_{\sigma_{0}}\left(m\left(u_{0}, u\right)\right) \max _{\substack{0 \leq h<T_{1} \\
0 \leq \mathrm{m}<S_{1}}}\left\{\left|\frac{1}{h !} \mathrm{f}^{(h)}(\mathrm{m})\right|_{p_{0}}\right\} & \leq e^{15 n^{2} U_{0}}\left(e^{4 n U_{0}}\left\|u_{0}-\lambda(u)\right\|_{\bar{t}_{G}}, \sigma_{0}\right. \\
& \left.+\alpha^{-1}\right)\|s\|_{\bar{E}_{\alpha}, \sigma_{0}} \\
& \leq e^{\left(16 n^{2}-C_{0}^{3 / 2}\right) U_{0}}\|s\|_{\bar{E}_{\alpha}, \sigma_{0}} .
\end{aligned}
$$

Nous sommes maintenant en mesure d'appliquer le lemme d'interpolation 5.26, qui, via la majoration $|\mathrm{f}(m)|_{p_{0}} \leq|\mathrm{f}|_{\mathrm{r}}$, permet de majorer $\alpha_{\sigma_{0}}\left(m\left(u_{0}, u\right)\right)|\mathrm{f}(m)|_{p_{0}}$ par

$$
e^{2 n U_{0}\left(\log C_{0}+(\log \mathfrak{e})^{-1}\right)} \max \left\{e^{\left(17 n^{2}-C_{0}\right) U_{0}},\left(40 n C_{0}^{2}\right)^{2 n C_{0} U_{0}} e^{\left(16 n^{2}-C_{0}^{3 / 2}\right) U_{0}}\right\}\|s\|_{E_{\alpha}, \sigma_{0}},
$$

quantité plus petite que $e^{-(3 / 4) C_{0} U_{0}}\|s\|_{\bar{E}_{\alpha}, \sigma_{0}}$. En effet, dans le maximum, la première quantité $e^{17 n^{2}-C_{0}}$ l'emporte sur la seconde car

$$
C_{0}\left(2 n \log (40 n)+4 n \log C_{0}+1\right) \leq C_{0}^{3 / 2} \leq C_{0}^{3 / 2}+n^{2}
$$

grâce au choix de $C_{0}=(4 n)^{10 n}$. Grâce à $\mathfrak{e} \geq e$, on a alors

$$
2 n\left(\log C_{0}+(\log \mathfrak{e})^{-1}\right)+17 n^{2}-C_{0} \leq-3 C_{0} / 4
$$

En utilisant une nouvelle fois le lemme 5.25 pour passer de $\mathrm{f}(m)$ à $\frac{1}{\tau !} \mathrm{D}_{w}^{\tau} F_{s, \sigma_{0}}\left(m\left(u_{0}, u\right)\right)$, on trouve

$$
\alpha_{\sigma_{0}}\left(m\left(u_{0}, u\right)\right)\left|\frac{1}{\tau !} \mathrm{D}_{w}^{\tau} F_{s, \sigma_{0}}\left(m\left(u_{0}, u\right)\right)\right|_{p_{0}} \leq 2 e^{-(3 / 4) C_{0} U_{0}}\|s\|_{E_{\alpha}, \sigma_{0}} .
$$

Cette estimation est valide pour tout $\tau \in \mathbf{N}^{n}$ de longueur $\ell$ et, a priori, pour $(m, \tau) \notin \Upsilon$. Mais l'inégalité (35) montre qu'elle reste vraie pour $(m, \tau) \in \Upsilon$ puisque $\alpha^{-1} \leq \exp \left\{-C_{0}^{3 / 2} U_{0}\right\}$. La définition 5.18 du jet de $s$ (voir aussi (34)) conduit alors au résultat suivant.

Proposition 5.29. - On a $\left\|\operatorname{jet}_{W}^{\ell} s(m \mathrm{p})\right\|_{\overline{\mathfrak{J} \mathfrak{e t}, \sigma}} \leq \exp \left\{-C_{0} U_{0} / 2\right\}\|s\|_{\bar{E}_{\alpha}, \sigma}$ pour tout plongement $\sigma$ conjugué à $\sigma_{0}$.

5.12.2. Cas périodique. - Rappelons tout d'abord qu'il a été vu au $\S 5.5$ que le cas périodique n'est possible que lorsque $\sigma_{0}$ est complexe. Soit $\tau=\left(\tau_{1}, \ldots, \tau_{n}\right) \in \mathbf{N}^{n}$ de longueur $\ell$ et $\tau^{\prime}:=$ $\left(\tau_{1}, \ldots, \tau_{n-1}, 0\right)$. Soit $\mathrm{f}: \mathbf{C} \rightarrow \mathbf{C}$ l'application analytique définie par :

$$
\forall z \in \mathbf{C}, \quad \mathrm{f}(z)=\frac{1}{\tau^{\prime} !} \mathrm{D}_{w}^{\tau^{\prime}} F_{s, \sigma_{0}}(z(\lambda(u), u)) .
$$

Afin d'évaluer jet ${ }_{W}^{\ell} s(m \mathrm{p})$, nous allons donner une majoration fine de la dérivée $\mathrm{f}^{\left(\tau_{n}\right)}(m) / \tau_{n}$ ! au moyen du lemme d'interpolation 5.26. Observons tout d'abord que

$$
\left|\frac{1}{\tau_{n} !} \mathrm{f}^{\left(\tau_{n}\right)}(m)\right| \leq|\mathrm{f}|_{1+m}
$$

grâce à l'inégalité de Cauchy usuelle pour les fonctions holomorphes complexes. Le terme $|\mathrm{f}|_{1+m}$ peut être majoré au moyen du lemme d'interpolation [5.26 avec les paramètres suivants : $S_{1}:=$ $(n+t) S, \mathrm{r}:=2(n+t) S, \mathrm{R}:=2 \mathrm{re}, T_{1}:=T_{0}$ et $f:=\mathrm{f}$. Dans ce lemme d'interpolation intervient $|\mathrm{f}|_{\mathrm{R}}$ que l'on peut estimer en suivant la démonstration de la proposition 5.28 , dont les calculs ont 
été faits de telle sorte qu'ils conviennent encore ici. Par ailleurs, grâce à la formule (40), au lemme de comparaison 5.25, et par construction de $s$, les dérivées de f jusqu'à l'ordre $T_{0}$ en les nombres $z \in\{0, \ldots,(n+t) S\}$ sont petites comme dans l'estimation (42). En réalité une fois que l'on a observé qu'avec les choix de paramètres ci-dessus le produit $T_{1} S_{1}$ était presque le même que dans le cas précédent, tout se passe à l'identique, si ce n'est que l'on doit se servir de (44). Un dernier passage par le lemme de comparaison 5.25 permet alors d'obtenir in fine :

$$
\alpha_{\sigma_{0}}\left(m\left(u_{0}, u\right)\right)\left|\frac{1}{\tau !} \mathrm{D}_{w}^{\tau^{\prime}} \mathrm{D}_{(\lambda(u), u)}^{\tau_{n}} F_{s, \sigma_{0}}\left(m\left(u_{0}, u\right)\right)\right| \leq 2 \exp \left\{-(3 / 4) C_{0} U_{0}\right\}\|s\|_{E_{\alpha}, \sigma_{0}}
$$

(majoration valide pour tout $\tau=\left(\tau^{\prime}, \tau_{n}\right)$ de longueur $\ell$ ). Même si nous n'obtenons pas une estimation de $\frac{1}{\tau !} \mathrm{D}_{w}^{\tau} F_{s, \sigma_{0}}\left(m\left(u_{0}, u\right)\right)$, ceci n'a pas d'importance car ce qui nous intéresse est le jet d'ordre $\ell$ de $s$, qui lui ne dépend pas du choix de la base considérée pour le définir (voir le commentaire qui suit la définition 5.18). Choisissons la base $w:=\left(w_{1}, \ldots, w_{n-1},(\lambda(u), u)\right)$ dans la définition 5.18 Si $x \in W \otimes_{\sigma_{0}} \mathbf{C}$ a pour coordonnées $\left(x_{1}, \ldots, x_{n}\right)$ dans la base $w$ alors

$$
\forall i \in\{1, \ldots, n-1\}, \quad w_{i}^{\vee}(x)=x_{i}-x_{n} \frac{\mathfrak{u}_{i}}{\mathfrak{u}_{n}} \quad \text { et } \quad(\lambda(u), u)^{\vee}(x)=\frac{x_{n}}{\mathfrak{u}_{n}} .
$$

Par conséquent on a

$$
\forall i \in\{1, \ldots, n-1\}, \quad\left\|w_{i}^{\vee}\right\|_{\overline{t_{G}^{v}}, \sigma_{0}}=\left(1+\left(\frac{\left|\mathfrak{u}_{i}\right|}{\left|\mathfrak{u}_{n}\right|}\right)^{2}\right)^{1 / 2} \quad \text { et } \quad\left\|(\lambda(u), u)^{\vee}\right\|_{\overline{t_{G}^{v}}}, \sigma_{0}=\frac{1}{\left|\mathfrak{u}_{n}\right|} .
$$

De ce fait et de la majoration (45), l'on déduit

$$
\left\|\operatorname{jet}_{W}^{\ell} s(m \mathrm{p})\right\|_{\overline{\mathfrak{J e t}}, \sigma_{0}} \leq 2\left(\frac{\max \left\{1,\|(\lambda(u), u)\|_{\overline{t_{G}}, \sigma_{0}}\right\}}{\left|\mathfrak{u}_{n}\right|}\right)^{\ell} \exp \left\{-(3 / 4) C_{0} U_{0}\right\}\|s\|_{E_{\alpha}, \sigma_{0}} .
$$

L'hypothèse 5.27 et l'inégalité triangulaire donnent la majoration

$$
\|(\lambda(u), u)\|_{\overline{t_{G}}, \sigma_{0}} \leq 1+\left\|\left(u_{0}, u\right)\right\|_{\overline{t_{G}}, \sigma_{0}} \leq 1+\left\|u_{0}\right\|_{\overline{k^{n} / W_{0}}, \sigma_{0}}+\sum_{j=1}^{n}\left|u_{j}\right| .
$$

Avec la réduction (iii) du $\S 5.2$, on a $\left\|u_{0}\right\| \overline{k^{n} / W_{0}}, \sigma_{0} \leq 1$ et, comme

$$
\left|u_{j}\right| \leq\left(D \log a_{j}\right) / \mathfrak{e} \leq\left(\log a_{j}\right) \exp \{(\mathfrak{a} \log \mathfrak{e}) / D\}
$$

on en déduit

$$
\|(\lambda(u), u)\|_{\overline{t_{G}}, \sigma_{0}} \leq\left(1+\log \prod_{j=1}^{n} a_{j}\right) \exp \{(\mathfrak{a} \log \mathfrak{e}) / D\} \leq \exp \{(2 \mathfrak{a} \log \mathfrak{e}) / D\} .
$$

De plus, en vertu des propositions 5.8 et [5.9, on a $\left|\mathfrak{u}_{n}\right|^{-1} \leq C_{0} \mathfrak{a}\left(\begin{array}{c}n+t \\ n\end{array}\right) \widetilde{D}_{0}$. La proposition 5.4, (iv), permet de majorer $T \log \widetilde{D}_{0}$ par $\left(10 n \log C_{0}\right) U_{0}$. On trouve alors

$$
2\left(\frac{\max \left\{1,\|(\lambda(u), u)\|_{\bar{t}_{G}, \sigma_{0}}\right\}}{\left|\mathfrak{u}_{n}\right|}\right)^{\ell} \leq \exp \left\{\left(C_{0} / 4\right) U_{0}\right\} .
$$

Conjugué à l'inégalité (46), ce résultat montre que la proposition 5.29 reste vraie dans le cas périodique.

5.13. Conclusion. - La fin de la démonstration du théorème 5.1 repose sur le lemme 3.6 qui, compte tenu de la proposition 5.11, s'écrit ici sous la forme

$$
h\left(\operatorname{jet}_{W}^{\ell} s(m \mathrm{p})\right) \geq-\widehat{\mu}_{\max }(\overline{\mathfrak{J} e t}) .
$$

Nous allons montrer que cette minoration n'est pas compatible avec la majoration de la hauteur du jet que l'on peut trouver par le biais de l'hypothèse 5.27 En effet les propositions 5.23, 5.24 et 5.29 entraînent

$$
h\left(\operatorname{jet}_{W}^{\ell} s(m \mathrm{p})\right) \leq \frac{n U_{0}}{D}+T \log \left(4 D_{0}\right)-\frac{C_{0} U_{0}\left[k_{\sigma_{0}}: \mathbf{Q}_{p_{0}}\right]}{2 D}+h_{\bar{E}_{\alpha}}(s) .
$$


La proposition [5.4. (iv), qui permet de majorer $T \log \left(4 D_{0}\right)$ (en se rappelant que $D_{0} \leq \widetilde{D}_{0}$ car $x \leq 1$ ) et la construction de $s$ (proposition [5.17) conduisent à

$$
h\left(\operatorname{jet}_{W}^{\ell} s(m \mathbf{p})\right) \leq\left(n+10 n \log C_{0}-\frac{C_{0}}{2}+(4 n)^{4 n} C_{0}^{1 / 2}\right) \frac{\left[k_{\sigma_{0}}: \mathbf{Q}_{p_{0}}\right] U_{0}}{D} .
$$

Par ailleurs la pente maximale de $\overline{\mathfrak{J} \mathfrak{e t}}$ a été majorée par $7 n^{2} U_{0} / D$ (proposition 5.20 ). L'on s'aperçoit alors que le choix de $C_{0}$ met en contradiction (47) et (48). L'hypothèse 5.27 est donc fausse et $\log \left\|u_{0}-\lambda(u)\right\|_{\bar{t}_{G}, \sigma_{0}} \geq-\left(C_{0}^{3 / 2}+4 n\right) U_{0}$. La proposition 5.3 entraîne

$$
\log \max _{1 \leq i \leq t}\left|\Lambda_{i}\right|_{p_{0}} \geq-n^{3} \log b-\log \sqrt{n}-\left(C_{0}^{3 / 2}+4 n\right) U_{0} .
$$

Pour conclure et obtenir le théorème 5.1, on observe que

$$
U_{0} \leq C_{0}^{3 n+2}\left(C_{0}^{3}+2\right) \mathfrak{a}^{1 / t}(\log b+\mathfrak{a} \log \mathfrak{e}) \prod_{j=1}^{n}\left(1+\frac{D \log a_{j}}{\log \mathfrak{e}}\right)^{1 / t} .
$$

En effet, dans la formule (13) définissant $U_{0}$, on majore

$$
\left(\frac{C_{0}^{3 n-1} t ! \operatorname{card} \Sigma_{\mathrm{p}}(S)}{(n+t) !}\right)^{1 / t} \leq C_{0}^{3 n+2} \mathfrak{a}^{1 / t} .
$$

De plus, la quantité $\mathfrak{y}:=\log b+D \log S+S^{y} \log \mathfrak{e}$ vérifie

$$
\begin{aligned}
\mathfrak{y} & \leq \log b+3 D \log C_{0}+D \log \mathfrak{a}+C_{0}^{3} \mathfrak{a}^{y} \log \mathfrak{e} \\
& \leq\left(1+3 \log C_{0}\right) \log b+\left(C_{0}^{3}+2\right) \mathfrak{a} \log \mathfrak{e} \quad \text { (proposition [5.4, (iii)) } \\
& \leq\left(C_{0}^{3}+2\right)(\log b+\mathfrak{a} \log \mathfrak{e}),
\end{aligned}
$$

ce qui donne (49). Le théorème 5.1 découle alors de

$$
C_{0}^{3 n+2}\left(C_{0}^{3 / 2}+4 n\right)\left(C_{0}^{3}+2\right)+n^{3} \leq C_{0}^{10 n}=(4 n)^{90 n^{2}}
$$

(on a majoré $n^{3} \log b+\log \sqrt{n}$ par $n^{3} \log (b \mathfrak{e})$ ). Si $t=1$ et $\beta_{1,0} \neq 0$, on a $y=0$. La majoration de $\mathfrak{y}$ ci-dessus est remplacée par

$$
\mathfrak{y} \leq\left(1+3 \log C_{0}\right)(\log b+\log \mathfrak{e}+D \log \mathfrak{a})
$$

qui se répercute dans (49). En majorant $1+3 \log C_{0}$ par $C_{0}^{3}+2$ on obtient alors le théorème [5.1] dans ce cas.

\section{BIBLIOGRAPHIE}

[AG] M. Ably et É. Gaudron. Approximation diophantienne sur les courbes elliptiques à multiplication complexe. C. R. Acad. Sci. Paris, 337 (Sér. I):629-634, 2003.

[BP] D. Bertrand et P. Philippon. Sous-groupes algébriques de groupes algébriques commutatifs. Illinois J. Math., 32(2):263-280, 1988.

[BV] E. Bombieri et J. VAaler. On Siegel's lemma. Invent. math., 73(1):11-32, 1983. Avec un addendum : ibid. $75(2): 377,1984$.

[Bo] J.-B. Bost. Périodes et isogénies des variétés abéliennes sur les corps de nombres (d'après D. Masser et G. Wüstholz). Séminaire Bourbaki. Volume 237 d'Astérisque, 115-161. Société Mathématique de France, 1996.

[Br] S. BRuiltet. D'une mesure d'approximation simultanée à une mesure d'irrationalité : le cas de $\Gamma(1 / 4)$ et $\Gamma(1 / 3)$. Acta Arith., 104(3):243-281, 2002.

[Da] S. DAvid. Minorations de formes linéaires de logarithmes elliptiques, volume 62 de Mémoire de la Société Mathématique de France. S. M. F., 1995.

[DH] S. David et N. HiRATA-Kohno. Linear forms in elliptic logarithms. J. reine angew. Math., 628:37-89, 2009.

[DP] S. DAvid et P. Philippon. Minorations des hauteurs normalisées des sous-variétés des tores. Ann. Scuola Norm. Sup. Pisa Cl. Sci., XXVIII(4):489-543, 1999. 
[Do] P. Dong. Minorations de combinaisons linéaires de logarithmes $p$-adiques de nombres algébriques. Dissertationes Math. (Rozprawy Mat.), 343, 97p., 1995. Publié par l'Institut de Mathématiques, Académie des Sciences de Pologne.

[G1] É. Gaudron. Mesures d'indépendance linéaire de logarithmes dans un groupe algébrique commutatif. Invent. Math., 162(1):137-188, 2005.

[G2] É. Gaudron. Formes linéaires de logarithmes effectives sur les variétés abéliennes. Ann. Sci. École Norm. Sup., 39(5):699-773, 2006.

[G3] É. Gaudron. Étude du cas rationnel de la théorie des formes linéaires de logarithmes. $J$. Number Theory, 127(2):220-261, 2007.

[G4] É. Gaudron. Pentes des fibrés vectoriels adéliques sur un corps global. Rend. Sem. Mat. Univ. Padova, 119:21-95, 2008.

[G5] É. Gaudron. Géométrie des nombres adélique et lemmes de Siegel généralisés. Manuscripta math., 130(2):159-182, 2009.

[GR1] É. Gaudron et G. RÉmond. Lemmes de Siegel d'évitement. Acta Arith., à paraître, 2012.

[GR2] É. Gaudron et G. RÉmond. Minima, pentes et algèbre tensorielle. Israel Journal of Math., à paraître, 2012.

[H1] N. HiRAta-Kohno. Formes linéaires de logarithmes de points algébriques sur les groupes algébriques. Invent. Math., 104:401-433, 1991.

[H2] N. Hirata-Kohno. Approximations simultanées sur les groupes algébriques commutatifs. Compos. Math., 86:69-96, 1993.

[Lo] J.H. Loxton. Some problems involving powers of integers. Acta Arith., 46(2):113-123, 1986.

[Ma] K. MAhLER. Lectures on transcendental numbers, volume 546 de Lecture Notes in Math. Springer-Verlag, Berlin, 1976.

[Ma] E.M. MatveEv. An explicit lower bound for a homogeneous rational linear form in logarithms of algebraic numbers II. Izv. Ross. Akad. NaukSer. Math, 64(6):125-180, 2000.

[Mi] M. Mignotte. Sur la résolution de systèmes linéaires en nombres entiers. Séminaire DelangePisot-Poitou 15e année (1973/74), Théorie des nombres, Fasc. 2, Exp. ${ }^{\circ} 16.5$ pages, Paris 1975. Disponible sur www.numdam.org.

[Ne] J. Neukirch. Algebraic number theory, volume 322 de Grundlehren der Mathematischen Wissenschaften. Springer-Verlag, 1999.

[PGS] C. Perez-Garcia et W.H. Schikhof. Locally convex spaces over non-Archimedean valued fields, volume 119 de Cambridge Studies in Advanced Mathematics. Cambridge University Press, 2010.

[Ph] P. Philippon. Nouveaux lemmes de zéros dans les groupes algébriques commutatifs. Rocky Mt. J. Math., 26(3):1069-1088, 1996.

[PW1] P. Philippon et M. WaLdschmidt. Formes linéaires de logarithmes sur les groupes algébriques commutatifs. Illinois J. Math., 32(2):281-314, 1988.

[PW2] P. Philippon et M. WALdschmidt. Formes linéaires de logarithmes simultanées sur les groupes algébriques commutatifs. Séminaire de Théorie des Nombres Paris 1986 - 87, volume 75 de Progress in Mathematics, pp. 313-347. Birkhäuser Boston, Inc., 1989. Édité par Catherine Goldstein.

[Ra] K. Ramachandra. A note on Baker's method. J. Austral. Math. Soc., 10:197-203, 1969.

[Ré] G. RÉmond. Sur les sous-variétés des tores. Compositio Math., 134(3):337-366, 2002.

[RU] G. RÉmond et F. URfels. Approximation diophantienne de logarithmes elliptiques padiques. J. Number Theory, 57(1):133-169, 1996.

[RT] D. Roy et J.L. Thunder. An absolute Siegel's lemma. J. Reine angew. Math., 476:1-26, 1996. Avec un addendum et un erratum, ibid., 508:47-51, 1999.

[Ro] D. Roy. Interpolation sur des perturbations d'ensembles produits. Bull. Soc. Math. France, 130(2):387-408, 2002.

[Se] J.-P. SERRE. Endomorphismes complètement continus des espaces de Banach p-adiques. Inst. Hautes Études Sci. Publ. Math., 12:69-85, 1962. 
[Si] C.L. SIEGEL. Über einige Anwendungen diophantischer Approximationen. Abhandlungen der Preußischen Akad. der Wissenschaften, Nr. 1, 70 S, 1929.

[T1] A. Thue. Om en generel i store hele tal uløsbar ligning. Kra. Videnskabens Selskabs Skrifter, Mat. Nat. Kl, 7:1-15, 1908.

[T2] A. THuE. Über Annäherungswerte algebraischer Zahlen. J. Reine Angew. Math., 135:284305, 1909.

[W1] M. WALdschmidt. Nombres transcendants, volume 402 de Lecture Notes in Math. SpringerVerlag, 1974.

[W2] M. Waldschmidt. A lower bound for linear forms in logarithms. Acta Arith., 37:257-283, 1980.

[W3] M. Waldschmidt. Diophantine Approximation On Linear Algebraic Groups, volume 326 de Grundlehren der mathematischen Wissenschaften. Springer-Verlag, 2000.

[Wü] G. Wüstholz. Algebraische punkte auf analytischen untergruppen algebraischer gruppen. Ann. of Math., 129(3):501-517, 1989.

[Yu] K. Yu. Report on p-adic logarithmic forms. A panorama of number theory or the view from Baker's garden (Zürich, 1999), 11-25. Cambridge Univ. Press. 2002.

[Zh] S. Zhang. Positive line bundles on arithmetic varieties. J. Amer. Math. Soc., 8(1):187-221, 1995. 